$$
\begin{array}{lllll}
\lrcorner & \lrcorner
\end{array}
$$

\title{
$L$-series of Artin stacks over finite fields
}

Shenghao Sun

\lrcorner

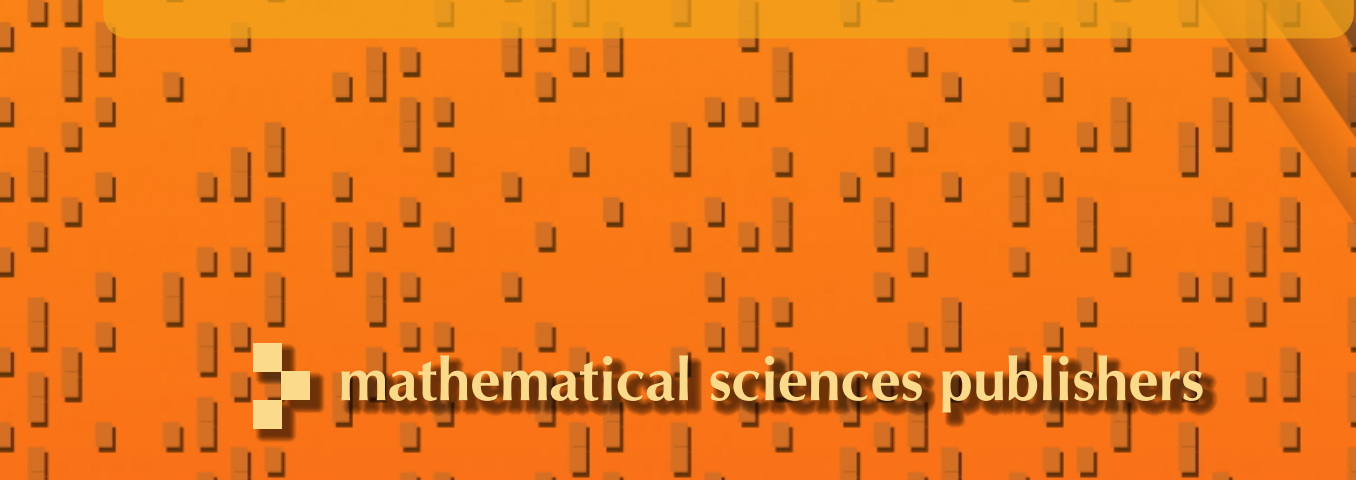

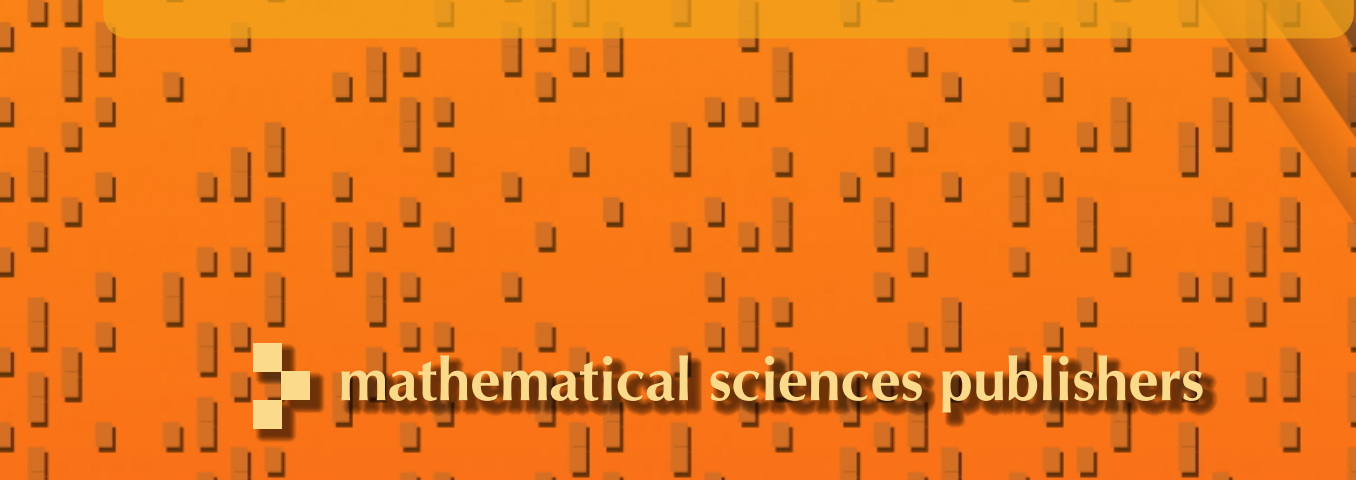

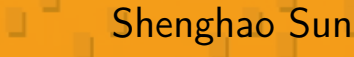




\title{
L-series of Artin stacks over finite fields
}

\author{
Shenghao Sun
}

\begin{abstract}
We develop the notion of stratifiability in the context of derived categories and the six operations for stacks. Then we reprove the Lefschetz trace formula for stacks, and give the meromorphic continuation of $L$-series (in particular, zeta functions) of $\mathbb{F}_{q}$-stacks. We also give an upper bound for the weights of the cohomology groups of stacks, and an "independence of $\ell$ " result for a certain class of quotient stacks.
\end{abstract}

\section{Introduction}

In topology there is the famous Lefschetz-Hopf trace formula, which roughly says that if $f: X \rightarrow X$ is an endomorphism of a compact connected oriented space $X$ with isolated fixed points, then the number of fixed points of $f$, counted with multiplicity, is equal to the alternating sum of the traces of $f^{*}$ on the singular cohomology groups $H^{i}(X, \mathbb{Q})$.

There is also a trace formula in algebraic geometry, for schemes over finite fields, due to Grothendieck. It says that if $X_{0}$ is a scheme over $\mathbb{F}_{q}$, separated and of finite type, and $F_{q}$ is the $q$-geometric Frobenius map, then

$$
\# X_{0}\left(\mathbb{F}_{q}\right)=\sum_{i=0}^{2 \operatorname{dim} X_{0}}(-1)^{i} \operatorname{Tr}\left(F_{q}, H_{c}^{i}\left(X, \overline{\mathbb{Q}}_{\ell}\right)\right),
$$

where $H_{c}^{i}\left(X, \overline{\mathbb{Q}}_{\ell}\right)$ is the $\ell$-adic cohomology with compact support. In fact he proved the trace formula for an arbitrary constructible sheaf, see [Grothendieck 1965, Verdier 1967, Deligne 1977].

Behrend conjectured the trace formula for smooth algebraic stacks over $\mathbb{F}_{q}$ in his thesis and [Behrend 1993], and proved it in [Behrend 2003]. However, he used ordinary cohomology and arithmetic Frobenius (rather than compact support cohomology and geometric Frobenius) to prove the "dual statement," probably because at that time the theory of dualizing complexes of algebraic stacks, as well as compact support cohomology groups of stacks, were not developed. Later Laszlo and Olsson [2008a; 2008b] developed the theory of the six operations for

MSC2010: primary 14F20; secondary 14F05, $19 \mathrm{~F} 27$.

Keywords: $l$-adic cohomology, algebraic stack, Lefschetz trace formula, $L$-function. 
algebraic stacks, which makes it possible to reprove the trace formula, and remove the smoothness assumption in Behrend's result. Also we will work with a fixed isomorphism of fields $\iota: \overline{\mathbb{Q}}_{\ell} \stackrel{\sim}{\longrightarrow} \mathbb{C}$, namely we will work with $\iota$-mixed complexes, rather than mixed ones, and this is a more general setting (see Remark 2.8.1).

Once we have the trace formula, we get a factorization of the zeta function into a possibly infinite product of $L$-factors, and from this one can deduce the meromorphic continuation of the zeta functions, generalizing a result of Behrend [1993, 3.2.4]. Furthermore, to locate the zeros and poles of the zeta functions, we give a result on the weights of cohomology groups of stacks.

We briefly mention the technical issues. As pointed out in [Behrend 2003], a big difference between schemes and stacks is the following. If

$$
f: X_{0} \rightarrow Y_{0}
$$

is a morphism of $\mathbb{F}_{q}$-schemes of finite type, and $K_{0} \in D_{c}^{b}\left(X_{0}, \overline{\mathbb{Q}}_{\ell}\right)$, then $f_{*} K_{0}$ and $f_{!} K_{0}$ are also bounded complexes. Since often we are mainly interested in the simplest case when $K_{0}$ is a sheaf concentrated in degree 0 (for instance, the constant sheaf $\overline{\mathbb{Q}}_{\ell}$ ), and $D_{c}^{b}$ is stable under $f_{*}$ and $f_{!}$, it is enough to consider $D_{c}^{b}$ only. But for a morphism

$$
f: \mathscr{X}_{0} \rightarrow \mathscr{Y}_{0}
$$

of $\mathbb{F}_{q}$-algebraic stacks of finite type, $f_{*}$ and $f_{\text {! }}$ do not necessarily preserve boundedness. For instance, the cohomology ring $H^{*}\left(B \mathbb{G}_{m}, \overline{\mathbb{Q}}_{\ell}\right)$ is the polynomial ring $\overline{\mathbb{Q}}_{\ell}[T]$ with $\operatorname{deg}(T)=2$. So for stacks we have to consider unbounded complexes, even if we are only interested in the constant sheaf $\overline{\mathbb{Q}}_{\ell}$. In order to define the trace of the Frobenius on cohomology groups, we need to consider the convergence of the complex series of the traces. This leads to the notion of an $\iota$-convergent complex of sheaves (see Definition 4.1).

Another issue is the following. In the scheme case one considers bounded complexes, and for any bounded complex $K_{0}$ on a scheme $X_{0}$, there exists a stratification of $X_{0}$ that "trivializes the complex $K_{0}$ " (that is, the restrictions of all cohomology sheaves $\mathscr{H}^{i} K_{0}$ to each stratum are lisse). But in the stack case we have to consider unbounded complexes, and in general there might be no stratification of the stack that trivializes every cohomology sheaf. This leads to the notion of a stratifiable complex of sheaves (see Definition 3.1). We need the stratifiability condition to control the dimensions of cohomology groups (see Lemma 3.10). All bounded complexes are stratifiable by Lemma $3.4(\mathrm{v})$.

Also we will have to impose the condition of $\iota$-mixedness, due to unboundedness. For bounded complexes on schemes, the trace formula can be proved without using this assumption, although the conjecture of Deligne [1980, 1.2.9] that all sheaves are $\iota$-mixed is proved by Laurent Lafforgue, see Remark 2.8.1. 
We briefly introduce the main results of this paper.

\section{Fixed point formula.}

Theorem 1.1. Let $\mathscr{L}_{0}$ be an Artin stack of finite type over $\mathbb{F}_{q}$, and let $\left[\mathscr{X}_{0}\left(\mathbb{F}_{q}\right)\right]$ be the set of isomorphism classes of the groupoid of $\mathbb{F}_{q}$-points of $\mathscr{X}_{0}$. Then the series

$$
\sum_{n \in \mathbb{Z}}(-1)^{n} \operatorname{Tr}\left(F_{q}, H_{c}^{n}\left(\mathscr{Q}, \overline{\mathbb{Q}}_{\ell}\right)\right),
$$

regarded as a complex series via $\iota$, is absolutely convergent, and its limit is "the number of $\mathbb{F}_{q}$-points of $\mathscr{X}_{0}$, namely

$$
\# \mathscr{L}_{0}\left(\mathbb{F}_{q}\right):=\sum_{x \in\left[\mathscr{Q}_{0}\left(\mathbb{F}_{q}\right)\right]} \frac{1}{\# \operatorname{Aut}_{x} \mathbb{F}_{q}} .
$$

Here $F_{q}$ denotes the $q$-geometric Frobenius. To generalize, one wants to impose some condition $(\mathrm{P})$ on complexes $K_{0} \in D_{c}^{-}\left(\mathscr{Q}_{0}, \overline{\mathbb{Q}}_{\ell}\right)$ such that:

(1) The condition $(\mathrm{P})$ is preserved by $f$ !

(2) If a complex $K_{0}$ satisfies (P), then the "naive local terms" are well-defined.

(3) Trace formula holds in this case.

The condition $(\mathrm{P})$ on $K_{0}$ turns out to be a combination of three parts: $\iota$-convergence (which implies (2) for $K_{0}$ ), $\iota$-mixedness and stratifiability (which, together with the first part, implies (2) for $f_{!} K_{0}$ ). See Theorem 4.2 for the general statement. These conditions are due to Behrend [2003].

Meromorphic continuation. The rationality in Weil conjecture was first proved by Dwork, namely the zeta function $Z\left(X_{0}, t\right)$ of every variety $X_{0}$ over $\mathbb{F}_{q}$ is a rational function in $t$. Later, this was reproved using the fixed point formula [Grothendieck 1965, Illusie 1977]. Following Behrend [1993, 3.2.3], we define the zeta functions of stacks as follows.

Definition 1.2. For an $\mathbb{F}_{q}$-algebraic stack $\mathscr{X}_{0}$ of finite type, define the zeta function

$$
Z\left(\mathscr{L}_{0}, t\right)=\exp \left(\sum_{v \geq 1} \frac{t^{v}}{v} \sum_{x \in\left[\mathscr{Q}_{0}\left(\mathbb{F}_{q} v\right)\right]} \frac{1}{\# \mathrm{Aut}_{x} \mathbb{F}_{q^{v}}}\right),
$$

as a formal power series in the variable $t$.

Notice that in general, the zeta function is not rational (see Section 7). Behrend $[1993,3.2 .4,3.2 .5]$ proved that if $\mathscr{X}_{0}$ is a smooth algebraic stack, and it is a quotient of an algebraic space by a linear algebraic group, then its zeta function $Z\left(\mathscr{X}_{0}, t\right)$ is a meromorphic function in the complex $t$-plane; if $\mathscr{L}_{0}$ is a smooth Deligne-Mumford stack, then $Z\left(\mathscr{X}_{0}, t\right)$ is a rational function. These results can be generalized as follows. 
Theorem 1.3. For every $\mathbb{F}_{q}$-algebraic stack $\mathscr{L}_{0}$ of finite type, its zeta function $Z\left(\mathscr{X}_{0}, t\right)$ defines a meromorphic function in the whole complex $t$-plane. If $\mathscr{X}_{0}$ is Deligne-Mumford, then $Z\left(\mathscr{X}_{0}, t\right)$ is a rational function.

See Proposition 7.3.1 and Theorem 8.1 for the general statement.

A theorem of weights. One of the main results in [Deligne 1980] is that if $X_{0}$ is an $\mathbb{F}_{q}$-scheme, separated and of finite type, and $\mathscr{F}_{0}$ is an $\iota$-mixed sheaf on $X_{0}$ of punctual $\iota$-weights $\leq w \in \mathbb{R}$, then for every $n$, the punctual $\iota$-weights of $H_{c}^{n}(X, \mathscr{F})$ are $\leq w+n$. The cohomology groups are zero unless $0 \leq n \leq 2 \operatorname{dim} X_{0}$. We will see in Remark 7.2.1 that the upper bound $w+n$ for the punctual $\iota$-weights does not work in general for algebraic stacks. We will give an upper bound that applies to all algebraic stacks. Deligne's upper bound of weights still applies to stacks for which all the automorphism groups are affine.

Theorem 1.4. Let $\mathscr{X}_{0}$ be an $\mathbb{F}_{q}$-algebraic stack of finite type, and let $\mathscr{F}_{0}$ be an ı-mixed sheaf on $\mathscr{X}_{0}$, with punctual ı-weights $\leq w$, for some $w \in \mathbb{R}$. Then the l-weights of $H_{c}^{n}(\mathscr{X}, \mathscr{F})$ are $\leq \operatorname{dim} \mathscr{X}_{0}+\frac{n}{2}+w$, and they are congruent $\bmod \mathbb{Z}$ to weights that appear in $\mathscr{F}_{0}$. If $n>2 \operatorname{dim} \mathscr{X}_{0}$, then $H_{c}^{n}(\mathscr{X},-)=0$ on sheaves. If for all points $\bar{x} \in \mathscr{X}(\mathbb{F})$ in the support of $\mathscr{F}$, the automorphism group schemes Aut $_{\bar{x}}$ are affine, then the l-weights of $H_{c}^{n}(\mathscr{X}, \mathscr{F})$ are $\leq n+w$.

Organization. In Section 2 we review some preliminaries on derived categories of $\ell$-adic sheaves on algebraic stacks over $\mathbb{F}_{q}$ and $\iota$-mixed complexes, and show that $\iota$-mixedness is stable under the six operations.

In Section 3 we develop the notion of stratifiable complexes in the context of Laszlo and Olsson's $\ell$-adic derived categories, and prove its stability under the six operations.

In Section 4 we discuss convergent complexes, and show that they are preserved by $f !$. In Section 5 we prove the trace formula for stacks. These two theorems are stated and proved in [Behrend 2003] in terms of ordinary cohomology and arithmetic Frobenius, and the proof we give here uses geometric Frobenius.

In Section 6 we discuss convergence of infinite products of formal power series, which will be used in the proof of the meromorphic continuation. In Section 7 we give some examples of zeta functions of stacks, and give the functional equation of the zeta functions and independence of $\ell$ of Frobenius eigenvalues for proper varieties with quotient singularities in Proposition 7.3.2.

In Section 8 and Section 9, we prove the meromorphic continuation and the weight theorem respectively. Finally in Section 10 we discuss "independence of $\ell$ " for stacks, and prove Proposition 10.5 that for the quotient stack $\left[X_{0} / G_{0}\right]$, where $X_{0}$ is a proper smooth variety and $G_{0}$ is a linear algebraic group acting on $X_{0}$, the Frobenius eigenvalues on its cohomology groups are independent of $\ell$. 


\section{Notation and conventions.}

1.5.1. We fix a prime power $q=p^{a}$ and an algebraic closure $\mathbb{F}$ of the finite field $\mathbb{F}_{q}$ with $q$ elements. Let $F$ or $F_{q}$ be the $q$-geometric Frobenius, namely the $q$-th root automorphism on $\mathbb{F}$. Let $\ell$ be a prime number, $\ell \neq p$, and fix an isomorphism of fields $\overline{\mathbb{Q}}_{\ell} \stackrel{\iota}{\longrightarrow} \mathbb{C}$. For simplicity, let $|\alpha|$ denote the complex absolute value $|\iota \alpha|$, for $\alpha \in \overline{\mathbb{Q}}_{\ell}$.

1.5.2. In this paper, by an Artin stack (or an algebraic stack) over a base scheme $S$, we mean an $S$-algebraic stack in the sense of M. Artin [Laumon and Moret-Bailly 2000, 4.1] of finite type. When we want the more general setting of Artin stacks locally of finite type, we will mention that explicitly.

1.5.3. Objects over $\mathbb{F}_{q}$ will be denoted with an index 0 . For instance, $\mathscr{X}_{0}$ will denote an $\mathbb{F}_{q}$-Artin stack; if $\mathscr{F}_{0}$ is a lisse-étale sheaf (or more generally, a Weil sheaf 2.4) on $\mathscr{X}_{0}$, then $\mathscr{F}$ denotes its inverse image on $\mathscr{X}:=\mathscr{X}_{0} \otimes_{\mathbb{F}_{q}} \mathbb{F}$.

1.5.4. For a field $k$, let $\mathrm{Gal} k$ denote its absolute Galois group $\mathrm{Gal}\left(k^{\mathrm{sep}} / k\right)$. By a variety over $k$ we mean a separated reduced $k$-scheme of finite type. Let $W\left(\mathbb{F}_{q}\right)$ be the Weil group $F_{q}^{\mathbb{Z}}$ of $\mathbb{F}_{q}$.

1.5.5. For a profinite group $H$, by $\overline{\mathbb{Q}}_{\ell}$-representations of $H$ we always mean finitedimensional continuous representations (see [Deligne 1980], 1.1.6), and denote by $\operatorname{Rep}_{\overline{\mathbb{Q}}_{\ell}} H$ the category of such representations.

1.5.6. For a scheme $X$, we denote by $|X|$ the set of its closed points. For a category $\mathscr{C}$ we write $[\mathscr{C}$ ] for the collection of isomorphism classes of objects in $\mathscr{C}$. For example, if $v \geq 1$ is an integer, then $\left[\mathscr{C}_{0}\left(\mathbb{F}_{q^{v}}\right)\right]$ denotes the set of isomorphism classes of $\mathbb{F}_{q^{v}}$-points of the stack $\mathscr{L}_{0}$. This is a finite set.

For $x \in \mathscr{X}_{0}\left(\mathbb{F}_{q^{v}}\right)$ we will write $k(x)$ for the field $\mathbb{F}_{q^{v}}$. For an $\mathbb{F}_{q^{-}}$-scheme $X_{0}$ (always of finite type) and $x \in\left|X_{0}\right|$, we denote by $k(x)$ the residue field of $x$. In both cases, let $d(x)$ be the degree of the field extension $\left[k(x): \mathbb{F}_{q}\right]$, and let $N(x)=q^{d(x)}=\# k(x)$. Also in both cases let $x: \operatorname{Spec} \mathbb{F}_{q^{v}} \rightarrow \mathscr{X}_{0}$ (or $X_{0}$ ) be the natural map $(v=d(x))$, although in the second case the map is defined only up to an automorphism in $\operatorname{Gal}\left(k(x) / \mathbb{F}_{q}\right)$. Given a $K_{0} \in D_{c}\left(\mathscr{X}_{0}, \overline{\mathbb{Q}}_{\ell}\right)$ (see Section 2), the pullback $x^{*} K_{0} \in D_{c}\left(\operatorname{Spec} k(x), \overline{\mathbb{Q}}_{\ell}\right)=D_{c}\left(\operatorname{Rep}_{\overline{\mathbb{Q}}_{\ell}} \operatorname{Gal} k(x)\right)$ gives a complex $K_{\bar{x}}$ of representations of $\mathrm{Gal} k(x)$, and we let $F_{x}$ be the geometric Frobenius generator $F_{q^{d(x)}}$ of this group, called "the local Frobenius".

1.5.7. Let $V$ be a finite dimensional $\overline{\mathbb{Q}}_{\ell}$-vector space and $\varphi$ an endomorphism of $V$. For a function $f: \overline{\mathbb{Q}}_{\ell} \rightarrow \mathbb{C}$, we denote by $\sum_{V, \varphi} f(\alpha)$ the sum of values of $f$ in $\alpha$, with $\alpha$ ranging over all the eigenvalues of $\varphi$ on $V$ with multiplicities. For instance, $\sum_{V, \varphi} \alpha=\operatorname{Tr}(\varphi, V)$. 
A $0 \times 0$-matrix has trace 0 and determinant 1 . For $K \in D_{c}^{b}\left(\overline{\mathbb{Q}}_{\ell}\right)$ and an endomorphism $\varphi$ of $K$, we define, following Deligne [1977],

$$
\begin{aligned}
\operatorname{Tr}(\varphi, K) & :=\sum_{n \in \mathbb{Z}}(-1)^{n} \operatorname{Tr}\left(H^{n}(\varphi), H^{n}(K)\right), \quad \text { and } \\
\operatorname{det}(1-\varphi t, K) & :=\prod_{n \in \mathbb{Z}} \operatorname{det}\left(1-H^{n}(\varphi) t, H^{n}(K)\right)^{(-1)^{n}} .
\end{aligned}
$$

For unbounded complexes $K$ we use similar notations if the series (respectively the infinite product) converges (converges term by term; see Definition 6.2).

1.5.8. For a map $f: X \rightarrow Y$ and a sheaf $\mathscr{F}$ on $Y$, we sometimes write $H^{n}(X, \mathscr{F})$ for $H^{n}\left(X, f^{*} \mathscr{F}\right)$. We write $H^{n}(\mathscr{X})$ for $H^{n}\left(\mathscr{X}, \overline{\mathbb{Q}}_{\ell}\right)$, and $h^{n}\left(\mathscr{X}, \mathscr{F}_{\mathcal{F}}\right)$ for $\operatorname{dim} H^{n}(\mathscr{X}, \mathscr{F})$, and similarly for $H_{c}^{n}(\mathscr{X})$ and $h_{c}^{n}(\mathscr{X}, \mathscr{F})$.

1.5.9. For an $\mathbb{F}_{q}$-algebraic stack $\mathscr{X}_{0}$ and a Weil complex $K_{0}$ on $\mathscr{X}_{0}$, by $R \Gamma\left(\mathscr{X}_{0}, K_{0}\right)$ (respectively $R \Gamma_{c}\left(\mathscr{Q}_{0}, K_{0}\right)$ ) we mean $R a_{*} K_{0}$ (respectively $R a_{!} K_{0}$ ), where the morphism $a: \mathscr{X}_{0} \rightarrow \operatorname{Spec} \mathbb{F}_{q}$ is the structural map.

The derived functors $R f_{*}, R f_{!}, L f^{*}$ and $R f^{!}$are usually abbreviated as $f_{*}, f_{!}$, $f^{*}$ and $f^{!}$. But we reserve $\otimes$, Hom and Hom for the ordinary sheaf tensor product, sheaf Hom and Hom group, respectively, and use $\otimes^{L}, R \mathscr{H} o m$ and RHom for their derived functors.

\section{Derived category of $\ell$-adic sheaves and mixedness}

We briefly review the definition in [Laszlo and Olsson 2008a; 2008b] for derived category of $\ell$-adic sheaves on stacks. Then we show that $\iota$-mixedness is stable under the six operations. As a consequence of Lafforgue's result from Remark 2.8.1, this is automatic, but we still want to give a much more elementary argument. The proof works for mixed complexes as well, see Remark 2.12. One can also generalize the structure theorem of $\iota$-mixed sheaves in [Deligne 1980] to algebraic stacks as in Remark 2.7.1.

2.1. Let $\Lambda$ be a complete discrete valuation ring with maximal ideal $\mathfrak{m}$ and residual characteristic $\ell$. Let $\Lambda_{n}=\Lambda / \mathfrak{m}^{n+1}$, and let $\Lambda_{\bullet}$ be the pro-ring $\left(\Lambda_{n}\right)_{n}$. We take the base scheme $S$ to be a scheme that satisfies the following condition:

(LO): $S$ is noetherian, affine, excellent, finite-dimensional, in which $\ell$ is invertible, and all $S$-schemes of finite type have finite $\ell$-cohomological dimension. We denote by $\mathscr{X}, \mathscr{Y}, \ldots$ Artin stacks locally of finite type over $S$.

Consider the ringed topos $\mathscr{A}=\mathscr{A}(\mathscr{C}):=\operatorname{Mod}\left(\mathscr{C}_{\text {lis-êt }}^{\mathbb{N}}, \Lambda_{\bullet}\right)$ of projective systems $\left(M_{n}\right)_{n}$ of $\mathrm{Ab}\left(\mathscr{X}_{\text {lis-ét }}\right)$ such that $M_{n}$ is a $\Lambda_{n}$-module for each $n$, and the transition maps are $\Lambda$-linear. An object $M \in \mathscr{A}$ is said to be $A R$-null, if there exists an integer $r>0$ such that for every integer $n$, the composed map $M_{n+r} \rightarrow M_{n}$ is the zero 
map. A complex $K$ in $\mathscr{A}$ is called $A R$-null, if all cohomology systems $\mathscr{H}^{i}(K)$ are AR-null; it is called almost AR-null, if for every $U$ in Lis-ét( $\mathscr{C}$ ) (assumed to be of finite type over $S)$, the restriction of $\mathscr{H}^{i}(K)$ to $\operatorname{Et}(U)$ is AR-null. Let $\mathscr{D}(\mathscr{A})$ be the ordinary derived category of $\mathscr{A}$. See [Laumon and Moret-Bailly 2000, 18.1.4] for the definition of constructible sheaves on $\mathscr{X}_{\text {lis-ét. }}$.

Definition 2.2. An object $M=\left(M_{n}\right)_{n} \in \mathscr{A}$ is adic if all the $M_{n}$ 's are constructible, and for every $n$, the natural map

$$
\Lambda_{n} \otimes_{\Lambda_{n+1}} M_{n+1} \rightarrow M_{n}
$$

is an isomorphism. It is called almost adic if all the $M_{n}$ 's are constructible, and for every object $U$ in Lis-ét( $\mathscr{X})$, the restriction $\left.M\right|_{U}$ is AR-adic, that is, there exists an adic $N_{U} \in \operatorname{Mod}\left(U_{\mathrm{et}}^{\mathbb{N}}, \Lambda_{\bullet}\right)$ and a morphism $\left.N_{U} \rightarrow M\right|_{U}$ with $A R$-null kernel and cokernel.

A complex $K$ in $\mathscr{A}$ is a $\lambda$-complex if $\mathscr{H}^{i}(K) \in \mathscr{A}$ are almost adic for all $i$. Let $\mathscr{D}_{c}(\mathscr{A})$ be the full triangulated subcategory of $\mathscr{D}(\mathscr{A})$ consisting of $\lambda$-complexes, and let $D_{c}(\mathscr{X}, \Lambda)$ be the quotient of $\mathscr{D}_{c}(\mathscr{A})$ by the thick full subcategory of those which are almost AR-null. This is called the derived category of $\Lambda$-adic sheaves on $\mathscr{X}$.

Remark 2.2.1. (i) $D_{c}(\mathscr{X}, \Lambda)$ is a triangulated category with a natural $t$-structure, and its heart is the quotient of the category of almost adic systems in $\mathscr{A}$ by the thick full subcategory of almost AR-null systems. One can use this $t$-structure to define the subcategories $D_{c}^{\dagger}(\mathscr{X}, \Lambda)$ for $\dagger= \pm, b$.

If $\mathscr{X} / S$ is of finite type (in particular, quasi-compact), it is clear that $K \in \mathscr{D}_{\text {cart }}(\mathscr{A})$ is AR-null if it is almost AR-null. Also if $M \in \mathscr{A}$ is almost adic, the adic system $N_{U}$ and the map $\left.N_{U} \rightarrow M\right|_{U}$ in the definition above are unique up to unique isomorphism, for each $U$, so by [Laumon and Moret-Bailly 2000, 12.2.1] they give an adic system $N$ of Cartesian sheaves on $\mathscr{X}_{\text {lis-ét, }}$ and an AR-isomorphism $N \rightarrow M$. This shows that an almost adic system is AR-adic, and it is clear [Illusie 1977, p. 234] that the natural functor

$$
\Lambda-\operatorname{Sh}(\mathscr{X}) \rightarrow \text { heart } D_{c}(\mathscr{X}, \Lambda)
$$

is an equivalence of categories, where $\Lambda$-Sh $(\mathscr{X})$ denotes the category of $\Lambda$-adic (in particular, constructible) systems.

(ii) $D_{c}(\mathscr{X}, \Lambda)$ is different from the ordinary derived category of $\operatorname{Mod}\left(\mathscr{L}_{\text {lis-ét }}, \Lambda\right)$ with constructible cohomology; the latter can be denoted by $\mathscr{D}_{c}(\mathscr{X}, \Lambda)$. Here $\operatorname{Mod}\left(\mathscr{L}_{\text {lis-ét }}, \Lambda\right)$ denotes the abelian category of $\Lambda_{\mathscr{L}}$-modules, not adic sheaves $\Lambda-\operatorname{Sh}(\mathscr{L})$.

(iii) When $S=$ Spec $k$ for $k$ a finite field or an algebraically closed field, and $\mathscr{X}=X$ is a separated $S$-scheme, [Laszlo and Olsson 2008b, 3.1.6] gives a natural 
equivalence of triangulated categories between $D_{c}^{b}(X, \Lambda)$ and Deligne's definition $\mathscr{D}_{c}^{b}(X, \Lambda)$ in [Deligne 1980, 1.1.2].

2.3. Let $\pi: \mathscr{X}_{\text {lis-ét }}^{\mathbb{N}} \rightarrow \mathscr{X}_{\text {lis-ét }}$ be the morphism of topoi where $\pi^{-1}$ takes a sheaf $F$ to the constant projective system $(F)_{n}$, and $\pi_{*}$ takes a projective system to the inverse limit. This morphism induces a morphism of ringed topoi

$$
\left(\pi^{*}, \pi_{*}\right):\left(\mathscr{C}_{\text {lis-ét }}^{\mathbb{N}}, \Lambda_{\bullet}\right) \rightarrow\left(\mathscr{X}_{\text {lis-ét }}, \Lambda\right) .
$$

The functor $R \pi_{*}: \mathscr{D}_{c}(\mathscr{A}) \rightarrow \mathscr{D}(\mathscr{X}, \Lambda)$ vanishes on almost AR-null objects [Laszlo and Olsson 2008b, 2.2.2], hence factors through $D_{c}(\mathscr{X}, \Lambda)$. In [ibid., 3.0.8], the normalization functor is defined to be

$$
K \mapsto \widehat{K}:=L \pi^{*} R \pi_{*} K: D_{c}(\mathscr{Q}, \Lambda) \rightarrow \mathscr{D}(\mathscr{A}) .
$$

This functor plays an important role in defining the six operations (ibid.). For instance:

- For $F \in D_{c}^{-}(\mathscr{X}, \Lambda)$ and $G \in D_{c}^{+}(\mathscr{X}, \Lambda), \operatorname{RHom}(F, G)$ is defined to be the image of $R_{\mathscr{H}} \operatorname{om}_{\Lambda}(\widehat{F}, \widehat{G})$ in $D_{c}(\mathscr{Q}, \Lambda)$.

- For $F, G \in D_{c}^{-}(\mathscr{X}, \Lambda)$, the derived tensor product $F \otimes^{L} G$ is defined to be the image of $\widehat{F} \otimes_{\Lambda .}^{L} \widehat{G}$.

- For a morphism $f: \mathscr{X} \rightarrow \mathscr{Y}$ and $F \in D_{c}^{+}(\mathscr{X}, \Lambda)$, the derived direct image $f_{*} F$ is defined to be the image of $f_{*}^{\mathbb{N}} \widehat{F}$.

Let $E_{\lambda}$ be a finite extension of $\mathbb{Q}_{\ell}$ with ring of integers $\mathbb{O}_{\lambda}$. Following Laszlo and Olsson [2008b] we define $D_{c}\left(\mathscr{X}, E_{\lambda}\right)$ to be the quotient of $D_{c}\left(\mathscr{X}, \mathscr{O}_{\lambda}\right)$ by the full subcategory consisting of complexes $K$ such that, for every integer $i$, there exists an integer $n_{i} \geq 1$ such that $\mathscr{H}^{i}(K)$ is annihilated by $\lambda^{n_{i}}$. Then we define

$$
D_{c}\left(\mathscr{X}, \overline{\mathbb{Q}}_{\ell}\right)=2-\operatorname{colim}_{E_{\lambda}} D_{c}\left(\mathscr{X}, E_{\lambda}\right),
$$

where $E_{\lambda}$ ranges over all finite subextensions of $\overline{\mathbb{Q}}_{\ell} / \mathbb{Q}_{\ell}$, and the transition functors are

$$
E_{\lambda^{\prime}} \otimes_{E_{\lambda}}-: D_{c}\left(\mathscr{X}, E_{\lambda}\right) \rightarrow D_{c}\left(\mathscr{X}, E_{\lambda^{\prime}}\right)
$$

for $E_{\lambda} \subset E_{\lambda^{\prime}}$.

2.4. From now on in this section, $S=\operatorname{Spec} \mathbb{F}_{q}$. We recall some notions of weights and mixedness from [Deligne 1980], generalized to $\mathbb{F}_{q}$-algebraic stacks.

2.4.1. Frobenius endomorphism. For an $\mathbb{F}_{q}$-scheme $X_{0}$, let $F_{X_{0}}: X_{0} \rightarrow X_{0}$ be the morphism that is identity on the underlying topological space and $q$-th power on the structure sheaf $O_{X_{0}}$; this is an $\mathbb{F}_{q}$-morphism. Let $F_{X}: X \rightarrow X$ be the induced $\mathbb{F}$-morphism $F_{X_{0}} \times \operatorname{id}_{\mathbb{F}}$ on $X=X_{0} \otimes \mathbb{F}$. 
By functoriality of the maps $F_{X_{0}}$, we can extend it to stacks. For an $\mathbb{F}_{q}$-algebraic stack $\mathscr{X}_{0}$, define $F_{\mathscr{X}_{0}}: \mathscr{X}_{0} \rightarrow \mathscr{X}_{0}$ to be such that for every $\mathbb{F}_{q}$-scheme $X_{0}$, the map

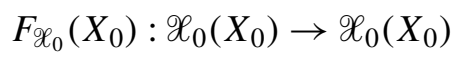

sends $x$ to $x \circ F_{X_{0}}$. We also define $F_{\mathscr{L}}: \mathscr{X} \rightarrow \mathscr{X}$ to be $F_{\mathscr{L}_{0}} \times \mathrm{id}_{\mathbb{F}}$. This morphism is a universal homeomorphism, hence $F_{\mathscr{L}}^{*}$ and $F_{\mathscr{Q} *}$ are quasi-inverse to each other, and $F_{\mathscr{L}}^{*} \simeq F_{\mathscr{L}}^{!}, F_{\mathscr{L} *} \simeq F_{\mathscr{L} !}$.

2.4.2. Weil complexes. A Weil complex $K_{0}$ on $\mathscr{L}_{0}$ is a pair $(K, \varphi)$, where $K$ is in $D_{c}\left(\mathscr{X}, \overline{\mathbb{Q}}_{\ell}\right)$ and $\varphi: F_{\mathscr{L}}^{*} K \rightarrow K$ is an isomorphism. A morphism of Weil complexes on $\mathscr{X}_{0}$ is a morphism of complexes on $\mathscr{X}$ commuting with $\varphi$. We also call $K_{0}$ a Weil sheaf if $K$ is a sheaf. Let $W\left(\mathscr{Q}_{0}, \overline{\mathbb{Q}}_{\ell}\right)$ be the category of Weil complexes on $\mathscr{X}_{0}$; it is a triangulated category with the standard $t$-structure, and its core is the category of Weil sheaves. There is a natural fully faithful triangulated functor

$$
D_{c}\left(\mathscr{X}_{0}, \overline{\mathbb{Q}}_{\ell}\right) \rightarrow W\left(\mathscr{X}_{0}, \overline{\mathbb{Q}}_{\ell}\right) .
$$

The usual six operations are well-defined on Weil complexes.

- Verdier duality. The Weil complex structure on $D_{\mathscr{L}} K$ is given by the inverse of the isomorphism

$$
D_{\mathscr{X}} K \stackrel{D \varphi}{\longrightarrow} D_{\mathscr{L}} F_{\mathscr{L}}^{*} K \stackrel{\sim}{\longrightarrow} F_{\mathscr{L}}^{*} D_{\mathscr{L}} K .
$$

- Tensor product. Let $K_{0}$ and $L_{0}$ be two Weil complexes such that $K \otimes^{L} L$ (which is $K \otimes L$ since they are of $\overline{\mathbb{Q}}_{\ell}$-coefficients) is constructible. This is the case when they are both bounded above. The Weil complex structure on $K \otimes L$ is given by

$$
F_{\mathscr{L}}^{*}(K \otimes L) \stackrel{\sim}{\longrightarrow} F_{\mathscr{L}}^{*} K \otimes F_{\mathscr{L}}^{*} L \stackrel{\varphi_{K} \otimes \varphi_{L}}{\longrightarrow} K \otimes L .
$$

- Pullback. This is clear:

$$
F_{\mathscr{Q}}^{*} f^{*} K \stackrel{\sim}{\longrightarrow} f^{*} F_{0 \mathrm{y}}^{*} K \stackrel{f^{*} \varphi}{\longrightarrow} f^{*} K .
$$

Here $f: \mathscr{X}_{0} \rightarrow \mathscr{Y}_{0}$ is an $\mathbb{F}_{q}$-morphism and $(K, \varphi)$ is a Weil complex on $\mathscr{Y}_{0}$.

- Pushforward. Let $f: \mathscr{X}_{0} \rightarrow \mathscr{Y}_{0}$ and $K_{0} \in W^{+}\left(\mathscr{L}_{0}, \overline{\mathbb{Q}}_{\ell}\right)$. The Weil complex structure on $f_{*} K$ is given by

$$
F_{\dddot{9}}^{*} f_{*} K \longrightarrow f_{*} F_{\mathscr{Q}}^{*} K \stackrel{f^{*} \varphi}{\longrightarrow} f_{*} K,
$$

where the first arrow is an isomorphism, because it is adjoint to

$$
f_{*} K \rightarrow F_{9 *} f_{*} F_{\mathscr{L}}^{*} K \simeq f_{*} F_{\mathscr{L} *} F_{\mathscr{L}}^{*} K
$$

obtained by applying $f_{*}$ to the adjunction morphism $K \rightarrow F_{\mathscr{L} *} F_{\mathscr{L}}^{*} K$, which is an isomorphism. 
- The remaining cases $f^{!}, f$ ! and $R \mathscr{H}$ om follow from the previous cases.

In this article, when discussing stacks over $\mathbb{F}_{q}$, by a "sheaf" or "complex of sheaves," we usually mean a "Weil sheaf" or "Weil complex," whereas a "lisseétale sheaf or complex" will be an ordinary constructible $\overline{\mathbb{Q}}_{\ell}$-sheaf or complex on the lisse-étale site of $\mathscr{X}_{0}$.

For $x \in \mathscr{L}_{0}\left(\mathbb{F}_{q^{v}}\right)$, it is a fixed point of $F_{\mathscr{X}}^{v}$, hence there is a "local Frobenius automorphism" $F_{x}: K_{\bar{x}} \rightarrow K_{\bar{x}}$ for every Weil complex $K_{0}$, defined to be

$$
K_{\bar{x}} \simeq K_{F_{\mathscr{C}}(\bar{x})}=\left(F_{\mathscr{L}}^{*} K\right)_{\bar{x}} \stackrel{\varphi}{\longrightarrow} K_{\bar{x}} .
$$

2.4.3. $\iota$-Weights and $\iota$-mixedness. Recall that $\iota$ is a fixed isomorphism $\overline{\mathbb{Q}}_{\ell} \rightarrow \mathbb{C}$. For $\alpha \in \overline{\mathbb{Q}}_{\ell}^{*}$, let $w_{q}(\alpha):=2 \log _{q}|\iota \alpha|$, called the $\iota$-weight of $\alpha$ relative to $q$. For a real number $\beta$, a sheaf $\mathscr{F}_{0}$ on $\mathscr{X}_{0}$ is said to be punctually -pure of weight $\beta$, if for every integer $v \geq 1$ and every $x \in \mathscr{L}_{0}\left(\mathbb{F}_{q^{v}}\right)$, and every eigenvalue $\alpha$ of $F_{x}$ acting on $\mathscr{F}_{\bar{x}}$, we have $w_{N(x)}(\alpha)=\beta$. We say $\mathscr{F}_{0}$ is $\iota$-mixed if it has a finite filtration with successive quotients punctually $\iota$-pure, and the weights of these quotients are called the punctual $\iota$-weights of $\mathscr{F}_{0}$. A complex $K_{0} \in W\left(\mathscr{Q}_{0}, \overline{\mathbb{Q}}_{\ell}\right)$ is said to be $\iota$-mixed if all the cohomology sheaves $\mathscr{H}^{i} K_{0}$ are $\iota$-mixed. Let $W_{m}\left(\mathscr{L}_{0}, \overline{\mathbb{Q}}_{\ell}\right)$ (respectively $\left.D_{m}\left(\mathscr{X}_{0}, \overline{\mathbb{Q}}_{\ell}\right)\right)$ be the full subcategory of $\iota$-mixed complexes in $W\left(\mathscr{C}_{0}, \overline{\mathbb{Q}}_{\ell}\right)$ (respectively $\left.D_{c}\left(\mathscr{Q}_{0}, \overline{\mathbb{Q}}_{\ell}\right)\right)$.

One can also define punctually pure sheaves, mixed sheaves and mixed complexes for algebraic stacks.

2.4.4. Twists. For $b \in \overline{\mathbb{Q}}_{\ell}^{*}$, let $\overline{\mathbb{Q}}_{\ell}^{(b)}$ be the Weil sheaf on Spec $\mathbb{F}_{q}$ of rank one, where $F$ acts by multiplication by $b$. This is an étale sheaf if and only if $b$ is an $\ell$-adic unit [Deligne 1980, 1.2.7]. For an algebraic stack $\mathscr{X}_{0} / \mathbb{F}_{q}$, we also denote by $\overline{\mathbb{Q}}_{\ell}^{(b)}$ the inverse image on $\mathscr{X}_{0}$ of the above Weil sheaf via the structural map. If $\mathscr{F}_{0}$ is a sheaf on $\mathscr{X}_{0}$, we denote by $\mathscr{F}_{0}^{(b)}$ the tensor product $\mathscr{F}_{0} \otimes \overline{\mathbb{Q}}_{\ell}^{(b)}$, and say that $\mathscr{F}_{0}^{(b)}$ is deduced from $\mathscr{F}_{0}$ by a generalized Tate twist. Note that the operation $\mathscr{F}_{0} \mapsto \mathscr{F}_{0}^{(b)}$ adds the weights by $w_{q}(b)$. For an integer $d$, the usual Tate twist $\overline{\mathbb{Q}}_{\ell}(d)$ is $\overline{\mathbb{Q}}_{\ell}^{\left(q^{-d}\right)}$. We denote by $\langle d\rangle$ the operation $(d)[2 d]$ on complexes of sheaves, where $[2 d]$ means shifting $2 d$ to the left. Note that $\iota$-mixedness is stable under the operation $\langle d\rangle$.

Lemma 2.5. Let $\mathscr{X}_{0}$ be an $\mathbb{F}_{q}$-algebraic stack.

(i) If $\mathscr{F}_{0}$ is an 1 -mixed sheaf on $\mathscr{X}_{0}$, then so is every subquotient of $\mathscr{F}_{0}$.

(ii) If $0 \rightarrow \mathscr{F}_{0}^{\prime} \rightarrow \mathscr{F}_{0} \rightarrow \mathscr{F}_{0}^{\prime \prime} \rightarrow 0$ is an exact sequence of sheaves on $\mathscr{X}_{0}$, and $\mathscr{F}_{0}^{\prime}$ and $\mathscr{F}_{0}^{\prime \prime}$ are $\iota$-mixed, then so is $\mathscr{F}_{0}$.

(iii) The full subcategory $W_{m}\left(\mathscr{Q}_{0}, \overline{\mathbb{Q}}_{\ell}\right)$ (respectively $D_{m}\left(\mathscr{Q}_{0}, \overline{\mathbb{Q}}_{\ell}\right)$ ) of $W\left(\mathscr{Q}_{0}, \overline{\mathbb{Q}}_{\ell}\right)$ (respectively $D_{c}\left(\mathscr{Q}_{0}, \overline{\mathbb{Q}}_{\ell}\right)$ ) is a triangulated subcategory with induced standard $t$ structure. 
(iv) If $f$ is a morphism of $\mathbb{F}_{q}$-algebraic stacks, then $f^{*}$ on complexes of sheaves preserves ı-mixedness.

(v) If $j: \mathscr{U}_{0} \hookrightarrow \mathscr{L}_{0}$ is an open immersion and $i: \mathscr{L}_{0} \hookrightarrow \mathscr{X}_{0}$ is its complement, then $K_{0} \in W\left(\mathscr{Q}_{0}, \overline{\mathbb{Q}}_{\ell}\right)$ is ı-mixed if and only if $j^{*} K_{0}$ and $i^{*} K_{0}$ are $\iota$-mixed.

Proof. (i) If $\mathscr{F}_{0}$ is punctually $\iota$-pure of weight $\beta$, then so is every subquotient of it. Now suppose $\mathscr{F}_{0}$ is $\iota$-mixed and $\mathscr{F}_{0}^{\prime}$ is a subsheaf of $\mathscr{F}_{0}$. Let $W$ be a finite filtration

$$
0 \subset \cdots \subset \mathscr{F}_{0}^{i-1} \subset \mathscr{F}_{0}^{i} \subset \cdots \subset \mathscr{F}_{0}
$$

of $\mathscr{F}_{0}$ such that $\mathrm{Gr}_{i}^{W} \mathscr{F}_{0}:=\mathscr{F}_{0}^{i} / \mathscr{F}_{0}^{i-1}$ is punctually $\iota$-pure for every $i$. Let $W^{\prime}$ be the induced filtration $W \cap \mathscr{F}_{0}^{\prime}$ of $\mathscr{F}_{0}^{\prime}$. Then $\mathrm{Gr}_{i}^{W^{\prime}} \mathscr{F}_{0}^{\prime}$ is the image of

$$
\mathscr{F}_{0}^{i} \cap \mathscr{F}_{0}^{\prime} \subset \mathscr{F}_{0}^{i} \rightarrow \mathrm{Gr}_{i}^{W} \mathscr{F}_{0},
$$

so it is punctually $\iota$-pure. Let $\mathscr{F}_{0}^{\prime \prime}=\mathscr{F}_{0} / \mathscr{F}_{0}^{\prime}$ be a quotient of $\mathscr{F}_{0}$, and let $W^{\prime \prime}$ be the induced filtration of $\mathscr{F}_{0}^{\prime \prime}$, namely $\left(\mathscr{F}_{0}^{\prime \prime}\right)^{i}:=\mathscr{F}_{0}^{i} /\left(\mathscr{F}_{0}^{i} \cap \mathscr{F}_{0}^{\prime}\right)$. Then

$$
\mathrm{Gr}_{i}^{W^{\prime \prime}} \mathscr{F}_{0}^{\prime \prime}=\mathscr{F}_{0}^{i} /\left(\mathscr{F}_{0}^{i-1}+\mathscr{F}_{0}^{i} \cap \mathscr{F}_{0}^{\prime}\right),
$$

which is a quotient of $\mathscr{F}_{0}^{i} / \mathscr{F}_{0}^{i-1}=\mathrm{Gr}_{i}^{W} \mathscr{F}_{0}$, so it is punctually $\iota$-pure. Hence every subquotient of $\mathscr{F}_{0}$ is $\iota$-mixed.

(ii) Let $W^{\prime}$ and $W^{\prime \prime}$ be finite filtrations of $\mathscr{F}_{0}^{\prime}$ and $\mathscr{F}_{0}^{\prime \prime}$ respectively, such that $\mathrm{Gr}_{i}^{W^{\prime}} \mathscr{F}_{0}^{\prime}$ and $\mathrm{Gr}_{i}^{W^{\prime \prime}} \mathscr{F}_{0}^{\prime \prime}$ are punctually $\iota$-pure for every $i$. Then $W^{\prime}$ can be regarded as a finite filtration of $\mathscr{F}_{0}$ such that every member of the filtration is contained in $\mathscr{F}_{0}^{\prime}$, and $W^{\prime \prime}$ can be regarded as a finite filtration of $\mathscr{F}_{0}$ such that every member contains $\mathscr{F}_{0}^{\prime}$. Putting these two filtrations together, we get the desired filtration for $\mathscr{F}_{0}$.

(iii) Being a triangulated subcategory means [Deligne 1977, p. 271] that if the sequence $K_{0}^{\prime} \rightarrow K_{0} \rightarrow K_{0}^{\prime \prime} \rightarrow K_{0}^{\prime}[1]$ is an exact triangle in $W\left(\mathscr{X}_{0}, \overline{\mathbb{Q}}_{\ell}\right)$, and two of the three complexes are $\iota$-mixed, then so is the third. By the rotation axiom of a triangulated category, we can assume $K_{0}^{\prime}$ and $K_{0}^{\prime \prime}$ are $\iota$-mixed. We have the exact sequence

$$
\cdots \longrightarrow \mathscr{H}^{n} K_{0}^{\prime} \longrightarrow \mathscr{H}^{n} K_{0} \longrightarrow \mathscr{H}^{n} K_{0}^{\prime \prime} \longrightarrow \cdots,
$$

and by (i) and (ii) we see that $\mathscr{H}^{n} K_{0}$ is $\iota$-mixed.

$W_{m}\left(\mathscr{Q}_{0}, \overline{\mathbb{Q}}_{\ell}\right)$ has the induced $t$-structure because if $K_{0}$ is $\iota$-mixed, then its truncations $\tau_{\leq n} K_{0}$ and $\tau_{\geq n} K_{0}$ are $\iota$-mixed.

(iv) On sheaves, $f^{*}$ preserves stalks, so it is exact and preserves punctual $\iota$-purity on sheaves. Let $f: \mathscr{X}_{0} \rightarrow \mathscr{Y}_{0}$. Given an $\iota$-mixed sheaf $\mathscr{F}_{0}$ on $\mathscr{Y}_{0}$, let $W$ be a finite filtration of $\mathscr{F}_{0}$ such that each $\mathrm{Gr}_{i}^{W} \mathscr{F}_{0}$ is punctually $\iota$-pure. Then $f^{*} W$ gives a finite filtration of $f^{*} \mathscr{F}_{0}$ and each $\mathrm{Gr}_{i}^{f^{*} W} f^{*} \mathscr{F}_{0}=f^{*} \mathrm{Gr}_{i}^{W} \mathscr{F}_{0}$ is punctually $\iota$-pure. So the sheaf $f^{*} \mathscr{F}_{0}$ is $\iota$-mixed. For an $\iota$-mixed complex $K_{0}$ on $\mathscr{Y}_{0}$, note that $\mathscr{H}^{n}\left(f^{*} K_{0}\right)=f^{*} \mathscr{H}^{n}\left(K_{0}\right)$, hence $f^{*} K_{0}$ is $\iota$-mixed. 
(v) One direction follows from (iv). For the other direction, note that $j$ ! and $i_{*}$ are exact and preserve punctual $\iota$-purity on sheaves. If $\mathscr{F}_{0}$ is an $\iota$-mixed sheaf on $U_{0}$, with a finite filtration $W$ such that each $\mathrm{Gr}_{i}^{W} \mathscr{F}_{0}$ is punctually $\iota$-pure, then for the induced filtration $j ! W$ of $j ! \mathscr{F}_{0}$, we see that $\mathrm{Gr}_{i}^{j ! W} j_{!} \mathscr{F}_{0}=j$ ! $\mathrm{Gr}_{i}^{W} \mathscr{F}_{0}$ is punctually $\iota$-pure, so $j ! \mathscr{F}_{0}$ is $\iota$-mixed. For an $\iota$-mixed complex $K_{0}$ on $U_{0}$, we use that $\mathscr{H}^{n}\left(j_{!} K_{0}\right)=j ! \mathscr{H}^{n}\left(K_{0}\right)$. Similarly $i_{*}$ also preserves $\iota$-mixedness on complexes. Finally the result follows from (iii) and the exact triangle

$$
j ! j^{*} K_{0} \longrightarrow K_{0} \longrightarrow i_{*} i^{*} K_{0} \longrightarrow .
$$

To show that $\iota$-mixedness is stable under the six operations, we need to show that $\iota$-mixedness of complexes on stacks can be checked locally on their presentations. To descend a filtration on a presentation to the stack, we generalize the structure theorem of $\iota$-mixed sheaves to algebraic spaces. Recall the following theorem of Deligne [1980, 3.4.1].

Theorem 2.6. Let $\mathscr{F}_{0}$ be an $\iota$-mixed sheaf on a scheme $X_{0}$ over $\mathbb{F}_{q}$.

(i) $\mathscr{F}_{0}$ has a unique decomposition $\mathscr{F}_{0}=\bigoplus_{b \in \mathbb{R} / \mathbb{Z}} \mathscr{F}_{0}(b)$, called the decomposition according to the weights $\bmod \mathbb{Z}$, such that the punctual l-weights of $\mathscr{F}_{0}(b)$ are all in the coset $b$. This decomposition, in which almost all the $\mathscr{F}_{0}(b)$ are zero, is functorial in $\mathscr{F}_{0}$. Note that each $\mathscr{F}_{0}(b)$ is deduced by twist from an 1 -mixed sheaf with integer punctual weights.

(ii) If the punctual weights of $\mathscr{F}_{0}$ are integers and $\mathscr{F}_{0}$ is lisse, $\mathscr{F}_{0}$ has a unique finite increasing filtration $W$ by lisse subsheaves, called the filtration by punctual weights, such that $\mathrm{Gr}_{i}^{W} \mathscr{F}_{0}$ is punctually ı-pure of weight $i$. This filtration is functorial in $\mathscr{F}_{0}$. More precisely, any morphism between $\iota$-mixed lisse sheaves of integer punctual weights is strictly compatible with their filtrations by punctual weights.

(iii) If $\mathscr{F}_{0}$ is lisse and punctually $\iota$-pure, and $X_{0}$ is normal, then the sheaf $\mathscr{F}^{\text {on } X}$ is semisimple.

Remark 2.6.1. (i) If $\mathscr{C}$ is an abelian category and $\mathscr{D}$ is an abelian full subcategory of $\mathscr{C}$, and $C$ is an object in $\mathscr{D}$, then every direct summand of $C$ in $\mathscr{C}$ lies in $\mathscr{D}$ (or isomorphic to some object in $\mathscr{D})$. This is because the kernel of the composition

$$
A \oplus B \stackrel{p r_{A}}{\longrightarrow} A \stackrel{i_{A}}{\longrightarrow} A \oplus B
$$

is $B$. So direct summands of a lisse sheaf are lisse. If $\mathscr{F}_{0}$ in Theorem 2.6 (i) is lisse, then each $\mathscr{F}_{0}(b)$ is lisse.

(ii) If the $\overline{\mathbb{Q}}_{\ell}$-sheaf $\mathscr{F}_{0}$ is defined over some finite subextension $E_{\lambda}$ of $\overline{\mathbb{Q}}_{\ell} / \mathbb{Q}_{\ell}$, then its decomposition in Theorem 2.6 (i) and filtration in Theorem 2.6 (ii) are defined over $E_{\lambda}$. This is because the $E_{\lambda}$-action commutes with the Galois action. 
(iii) Deligne [1980] made the assumption that all schemes are separated, at least in order to use Nagata compactification to define $f_{!}$. After the work of Laszlo and Olsson [2008a, 2008b], one can remove this assumption, and many results in [Deligne 1980], for instance this one and (3.3.1), remain valid. For [Deligne 1980, 3.4.1] one can take a cover of a not necessarily separated scheme $X_{0}$ by open affines (which are separated), and use the functoriality to glue the decomposition or filtration on intersections.

Lemma 2.7. Let $X_{0}$ be an $\mathbb{F}_{q}$-algebraic space, and $\mathscr{F}_{0}$ an $\iota$-mixed sheaf on $X_{0}$.

(i) $\mathscr{F}_{0}$ has a unique decomposition $\mathscr{F}_{0}=\bigoplus_{b \in \mathbb{R} / \mathbb{Z}} \mathscr{F}_{0}(b)$, the decomposition according to the weights $\bmod \mathbb{Z}$, with the same property as in Theorem 2.6 (i). This decomposition is functorial in $\mathscr{F}_{0}$.

(ii) If the punctual $\iota$-weights of $\mathscr{F}_{0}$ are integers and $\mathscr{F}_{0}$ is lisse, $\mathscr{F}_{0}$ has a unique finite increasing filtration $W$ by lisse subsheaves, called the filtration by punctual weights, with the same property as in Theorem 2.6 (ii). This filtration is functorial in $\mathscr{F}_{0}$.

Proof. Let $P: X_{0}^{\prime} \rightarrow X_{0}$ be an étale presentation, and let $\mathscr{F}_{0}^{\prime}=P^{*} \mathscr{F}_{0}$, which is also $\iota$-mixed by Lemma 2.5 (iv). Let $X_{0}^{\prime \prime}$ be the fiber product

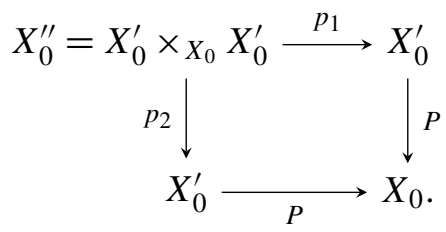

Then $X_{0}^{\prime \prime}$ is an $\mathbb{F}_{q}$-scheme of finite type.

(i) Applying Theorem 2.6 (i) to $\mathscr{F}_{0}^{\prime}$ we get a decomposition $\mathscr{F}_{0}^{\prime}=\bigoplus_{b \in \mathbb{R} / \mathbb{Z}} \mathscr{F}_{0}^{\prime}(b)$. For $j=1,2$, applying $p_{j}^{*}$ we get a decomposition

$$
p_{j}^{*} \mathscr{F}_{0}^{\prime}=\bigoplus_{b \in \mathbb{R} / \mathbb{Z}} p_{j}^{*} \mathscr{F}_{0}^{\prime}(b) .
$$

Since $p_{j}^{*}$ preserves weights, by the uniqueness in Theorem 2.6(i), this decomposition is the decomposition of $p_{j}^{*} \mathscr{F}_{0}^{\prime}$ according to the weights $\bmod \mathbb{Z}$. By the functoriality in Theorem 2.6 (i), the canonical isomorphism $\mu: p_{1}^{*} \mathscr{F}_{0}^{\prime} \rightarrow p_{2}^{*} \mathscr{F}_{0}^{\prime}$ takes the form $\bigoplus_{b \in \mathbb{R} / \mathbb{Z}} \mu_{b}$, where $\mu_{b}: p_{1}^{*} \mathscr{F}_{0}^{\prime}(b) \rightarrow p_{2}^{*} \mathscr{F}_{0}^{\prime}(b)$ is an isomorphism satisfying

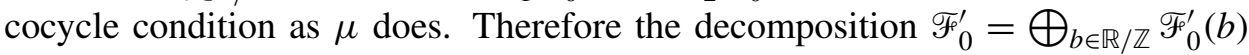
descends to a decomposition $\mathscr{F}_{0}=\bigoplus_{b \in \mathbb{R} / \mathbb{Z}} \mathscr{F}_{0}(b)$. We still need to show each direct summand $\mathscr{F}_{0}(b)$ is $\iota$-mixed.

Fix a coset $b$ and consider the summand $\mathscr{F}_{0}(b)$. Twisting it appropriately, we can assume that its inverse image $\mathscr{F}_{0}^{\prime}(b)$ is $\iota$-mixed with integer punctual $\iota$-weights. By Lemma $2.5(\mathrm{v})$ and noetherian induction, we can shrink $X_{0}$ to a nonempty 
open subspace and assume $\mathscr{F}_{0}(b)$ is lisse. Then $\mathscr{F}_{0}^{\prime}(b)$ is also lisse, and applying Theorem 2.6 (ii) we get a finite increasing filtration $W^{\prime}$ of $\mathscr{F}_{0}^{\prime}(b)$ by lisse subsheaves $\mathscr{F}_{0}^{\prime}(b)^{i}$, such that each $\operatorname{Gr}_{i}^{W^{\prime}} \mathscr{F}_{0}^{\prime}(b)$ is punctually $\iota$-pure of weight $i$. Pulling back this filtration via $p_{j}$, we get a finite increasing filtration $p_{j}^{*} W^{\prime}$ of $p_{j}^{*} \mathscr{F}_{0}^{\prime}(b)$, and since

$$
\operatorname{Gr}_{i}^{p_{j}^{*} W^{\prime}} p_{j}^{*} \mathscr{F}_{0}^{\prime}(b)=p_{j}^{*} \mathrm{Gr}_{i}^{W^{\prime}} \mathscr{F}_{0}^{\prime}(b)
$$

is punctually $\iota$-pure of weight $i$, it is the filtration by punctual weights given by Theorem 2.6 (ii), hence it is functorial. It follows that the canonical isomorphism $\mu_{b}: p_{1}^{*} \mathscr{F}_{0}^{\prime}(b) \rightarrow p_{2}^{*} \mathscr{F}_{0}^{\prime}(b)$ maps $p_{1}^{*} \mathscr{F}_{0}^{\prime}(b)^{i}$ isomorphically onto $p_{2}^{*} \mathscr{F}_{0}^{\prime}(b)^{i}$, satisfying cocycle condition. Therefore the filtration $W^{\prime}$ of $\mathscr{F}_{0}^{\prime}(b)$ descends to a filtration $W$ of $\mathscr{F}_{0}(b)$, and $P^{*} \mathrm{Gr}_{i}^{W} \mathscr{F}_{0}(b)=\mathrm{Gr}_{i}^{W^{\prime}} \mathscr{F}_{0}^{\prime}(b)$ is punctually -pure of weight $i$. Note that $P$ is surjective, so every point $x \in X_{0}\left(\mathbb{F}_{q^{v}}\right)$ can be lifted to a point $x^{\prime} \in X_{0}^{\prime}\left(\mathbb{F}_{q^{n v}}\right)$ after some base extension $\mathbb{F}_{q^{n v}}$ of $\mathbb{F}_{q^{v}}$. This shows $\mathrm{Gr}_{i}^{W} \mathscr{F}_{0}(b)$ is punctually $\iota$ pure of weight $i$, therefore $\mathscr{F}_{0}(b)$ is $\iota$-mixed. This proves the existence of the decomposition in (i).

For uniqueness, let $\mathscr{F}_{0}=\bigoplus \widetilde{\mathscr{F}}_{0}(b)$ be another decomposition with the desired property. Then their restrictions to $X_{0}^{\prime}$ are both equal to the decomposition of $\mathscr{F}_{0}^{\prime}$, which is unique Theorem 2.6(i), so they are both obtained by descending this decomposition, and so they are isomorphic, that is, for every coset $b$ there exists an isomorphism making the diagram commute:

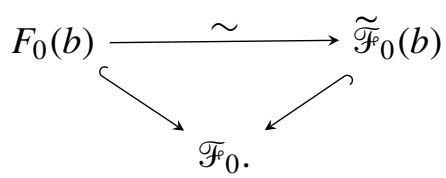

For functoriality, let $\mathscr{G}_{0}=\bigoplus \mathscr{G}_{0}(b)$ be another $\iota$-mixed sheaf with decomposition on $X_{0}$, and let $\varphi: \mathscr{F}_{0} \rightarrow \mathscr{G}_{0}$ be a morphism of sheaves. Pulling $\varphi$ back via $P$ we get a morphism $\varphi^{\prime}: \mathscr{F}_{0}^{\prime} \rightarrow \mathscr{G}_{0}^{\prime}$ on $X_{0}^{\prime}$, and the diagram

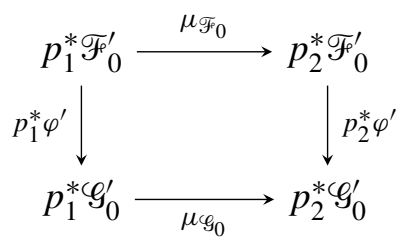

commutes. By Theorem 2.6 (i) $\varphi^{\prime}=\bigoplus \varphi^{\prime}(b)$ for morphisms $\varphi^{\prime}(b): \mathscr{F}_{0}^{\prime}(b) \rightarrow \mathscr{G}_{0}^{\prime}(b)$, and the diagram 


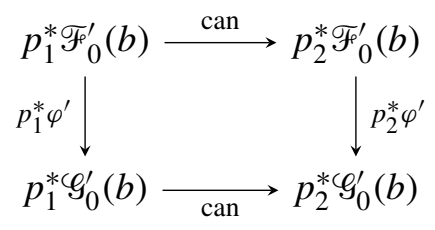

commutes for each $b$. It follows that the morphisms $\varphi^{\prime}(b)$ descend to morphisms $\varphi(b): \mathscr{F}_{0}(b) \rightarrow \mathscr{G}_{0}(b)$ such that $\varphi=\bigoplus \varphi(b)$.

(ii) The proof is similar to part (i). Applying Theorem 2.6 (ii) to $\mathscr{F}_{0}^{\prime}$ on $X_{0}^{\prime}$ we get a finite increasing filtration $W^{\prime}$ of $\mathscr{F}_{0}^{\prime}$ by lisse subsheaves $\mathscr{F}_{0}^{\prime i}$ with desired property. Pulling back this filtration via $p_{j}: X_{0}^{\prime \prime} \rightarrow X_{0}^{\prime}$ we get the filtration by punctual weights of $p_{j}^{* \mathscr{F}_{0}^{\prime}}$. By functoriality in Theorem 2.6 (ii), the canonical isomorphism $\mu: p_{1}^{* \mathscr{F}_{0}^{\prime}} \rightarrow p_{2}^{* \mathscr{F}_{0}^{\prime}}$ maps $p_{1}^{* \mathscr{F}_{0}^{\prime}}{ }_{0}^{i}$ isomorphically onto $p_{2}^{* \mathscr{F}_{0}^{\prime i}}$ satisfying cocycle condition, therefore the filtration $W^{\prime}$ descends to a finite increasing filtration $W$ of $\mathscr{F}_{0}$ by certain subsheaves $\mathscr{F}_{0}^{i}$. By Olsson $[2007,9.1]$ they are lisse subsheaves.

For uniqueness, if $\widetilde{W}$ is another filtration of $\mathscr{F}_{0}$ by certain subsheaves $\widetilde{\mathscr{F}}_{0}^{i}$ with desired property, then their restrictions to $X_{0}^{\prime}$ are both equal to the filtration $W^{\prime}$ by punctual weights, which is unique Theorem 2.6 (ii), so they are both obtained by descending this filtration $W^{\prime}$, and therefore they are isomorphic.

For functoriality, let $\mathscr{G}_{0}$ be another lisse $\iota$-mixed sheaf with integer punctual $\iota$ weights, and let $V$ be its filtration by punctual weights, and let $\varphi: \mathscr{F}_{0} \rightarrow \mathscr{G}_{0}$ be a morphism. Pulling $\varphi$ back via $P$ we get a morphism $\varphi^{\prime}: \mathscr{F}_{0}^{\prime} \rightarrow \mathscr{G}_{0}^{\prime}$ on $X_{0}^{\prime}$, and the diagram

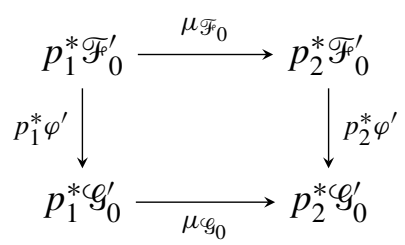

commutes. By Theorem 2.6 (ii) we have $\varphi^{\prime}\left(\mathscr{F}_{0}^{\prime i}\right) \subset \mathscr{G}_{0}^{\prime i}$, and the diagram

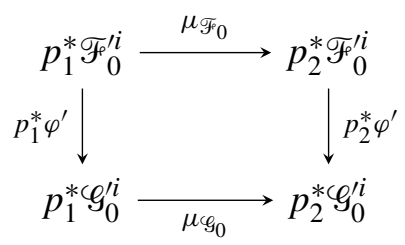

commutes for each $i$. Let $\varphi^{\prime i}: \mathscr{F}_{0}^{\prime i} \rightarrow \varphi_{0}^{\prime i}$ be the restriction of $\varphi^{\prime}$. Then they descend to morphisms $\varphi^{i}: \mathscr{F}_{0}^{i} \rightarrow \mathscr{G}_{0}^{i}$, which are restrictions of $\varphi$.

Remark 2.7.1. One can prove a similar structure theorem of $\iota$-mixed sheaves on algebraic stacks over $\mathbb{F}_{q}$ : the proof of Lemma 2.7 carries over verbatim to the 
case of algebraic stacks, except that for a presentation $X_{0}^{\prime} \rightarrow \mathscr{X}_{0}$, the fiber product $X_{0}^{\prime \prime}=X_{0}^{\prime} \times \mathscr{x}_{0} X_{0}^{\prime}$ may not be a scheme, so we use the case for algebraic spaces and replace every " 2.6 " in the proof by " 2.7 ". It turns out that Theorem 2.6 (iii) also holds for algebraic stacks, as a consequence of the proof of Theorem 1.4. As we will not use these results in this paper, we do not give the proof in detail here, but refer to [Sun 2012, 2.1].

Proposition 2.8. Let $\mathscr{X}_{0}$ be an $\mathbb{F}_{q}$-algebraic stack, and let $P: X_{0} \rightarrow \mathscr{X}_{0}$ be a presentation (that is, a smooth surjection with $X_{0}$ a scheme). Then a complex $K_{0} \in W\left(\mathscr{X}_{0}, \overline{\mathbb{Q}}_{\ell}\right)$ is l-mixed if and only if $P^{*} K_{0}$ (respectively $\left.P^{!} K_{0}\right)$ is l-mixed.

Proof. We consider $P^{*} K_{0}$ first. The "only if" part follows from Lemma 2.5 (iv). For the "if" part, since $P^{*}$ is exact on sheaves and so $\mathscr{H}^{i}\left(P^{*} K_{0}\right)=P^{*} \mathscr{H}^{i}\left(K_{0}\right)$, we reduce to the case when $K_{0}=\mathscr{F}_{0}$ is a sheaf. So we assume the sheaf $\mathscr{F}_{0}^{\prime}:=P^{*} \mathscr{F}_{0}$ on $X_{0}$ is $\iota$-mixed, and want to show $\mathscr{F}_{0}$ is also $\iota$-mixed. The proof is similar to the argument in Lemma 2.7.

Let $X_{0}^{\prime \prime}$ be the fiber product

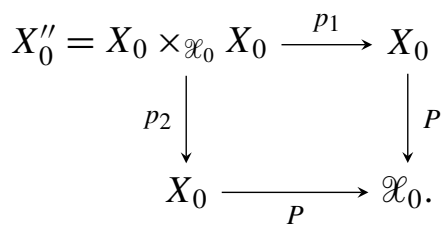

Then $X_{0}^{\prime \prime}$ is an algebraic space of finite type. Applying Theorem 2.6(i) to $\mathscr{F}_{0}^{\prime}$ we get a decomposition $\mathscr{F}_{0}^{\prime}=\bigoplus_{b \in \mathbb{R} / \mathbb{Z}} \mathscr{F}_{0}^{\prime}(b)$. For $j=1,2$, applying $p_{j}^{*}$ we get a decomposition

$$
p_{j}^{*} \mathscr{F}_{0}^{\prime}=\bigoplus_{b \in \mathbb{R} / \mathbb{Z}} p_{j}^{*} \mathscr{F}_{0}^{\prime}(b)
$$

which is the decomposition of $p_{j}^{*} \mathscr{F}_{0}^{\prime}$ according to the weights $\bmod \mathbb{Z}$. By the functoriality in Lemma 2.7 (i), the canonical isomorphism $\mu: p_{1}^{*} \mathscr{F}_{0}^{\prime} \rightarrow p_{2}^{*} \mathscr{F}_{0}^{\prime}$ takes the form $\bigoplus_{b \in \mathbb{R} / \mathbb{Z}} \mu_{b}$, where $\mu_{b}: p_{1}^{*} \mathscr{F}_{0}^{\prime}(b) \rightarrow p_{2}^{*} \mathscr{F}_{0}^{\prime}(b)$ is an isomorphism satisfying cocycle condition as $\mu$ does. Therefore the decomposition of $\mathscr{F}_{0}^{\prime}$ descends to a decomposition $\mathscr{F}_{0}=\bigoplus_{b \in \mathbb{R} / \mathbb{Z}} \mathscr{F}_{0}(b)$. The $\iota$-weights of the local Frobenius eigenvalues of $\mathscr{F}_{0}(b)$ at each point of $\mathscr{L}_{0}$ are in the coset $b$. Next we show that $\mathscr{F}_{0}(b)$ 's are $\iota$-mixed.

Replacing $\mathscr{F}_{0}$ by a direct summand $\mathscr{F}_{0}(b)$ and then twisting it appropriately, we may assume its inverse image $\mathscr{F}_{0}^{\prime}$ is $\iota$-mixed with integer punctual $\iota$-weights. By Lemma $2.5(\mathrm{v})$ we can shrink $\mathscr{X}_{0}$ to a nonempty open substack and assume $\mathscr{F}_{0}$ is lisse. Then $\mathscr{F}_{0}^{\prime}$ is also lisse, and applying Theorem 2.6 (ii) we get a finite increasing filtration $W^{\prime}$ of $\mathscr{F}_{0}^{\prime}$ by lisse subsheaves $\mathscr{F}_{0}^{\prime i}$, such that each $\operatorname{Gr}_{i}^{W^{\prime}} \mathscr{F}_{0}^{\prime}$ is punctually 
$\iota$-pure of weight $i$. Pulling back this filtration via $p_{j}$, we get a finite increasing filtration $p_{j}^{*} W^{\prime}$ of $p_{j}^{* \mathscr{F}_{0}^{\prime}}$, and since

$$
\mathrm{Gr}_{i}^{p_{j}^{*} W^{\prime}} p_{j}^{*} \mathscr{F}_{0}^{\prime}=p_{j}^{*} \mathrm{Gr}_{i}^{W^{\prime}} \mathscr{F}_{0}^{\prime}
$$

is punctually $\iota$-pure of weight $i$, it is the filtration by punctual weights given by Lemma 2.7 (ii). By functoriality, the canonical isomorphism $\mu: p_{1}^{*} \mathscr{F}_{0}^{\prime} \rightarrow p_{2}^{* \mathscr{F}_{0}^{\prime}}$ maps $p_{1}^{* \mathscr{F}_{0}}{ }_{0}^{i}$ isomorphically onto $p_{2}^{* \mathscr{F}_{0}^{\prime i}}$, satisfying cocycle condition. Therefore the filtration $W^{\prime}$ of $\mathscr{F}_{0}^{\prime}$ descends to a filtration $W$ of $\mathscr{F}_{0}$, and $P^{*} \mathrm{Gr}_{i}^{W} \mathscr{F}_{0}=\mathrm{Gr}_{i}^{W^{\prime}} \mathscr{F}_{0}^{\prime}$ is punctually $\iota$-pure of weight $i$. Since $\pi$ is surjective, $\operatorname{Gr}_{i}^{W} \mathscr{F}_{0}$ is also punctually $\iota$-pure of weight $i$, therefore $\mathscr{F}_{0}$ is $\iota$-mixed.

Next we consider $P^{!} K_{0}$. We know that $P$ is smooth of relative dimension $d$, for some function $d: \pi_{0}\left(X_{0}\right) \rightarrow \mathbb{N}$. Let $X_{0}^{0}$ be a connected component of $X_{0}$. Since $\pi_{0}\left(X_{0}\right)$ is finite, $X_{0}^{0}$ is both open and closed in $X_{0}$, so

$$
f: X_{0}^{0} \stackrel{j}{\longrightarrow} X_{0} \stackrel{P}{\longrightarrow} \mathscr{X}_{0}
$$

is smooth of relative dimension $d\left(X_{0}^{0}\right)$. Then $P^{*} K_{0}$ is $\iota$-mixed if and only if $f^{*} K_{0}=j^{*} P^{*} K_{0}$ is $\iota$-mixed for the inclusion $j$ of every connected component, if and only if $f^{!} K_{0}=f^{*} K_{0}\left\langle d\left(X_{0}^{0}\right)\right\rangle$ is $\iota$-mixed, if and only if $P^{!} K_{0}$ is $\iota$-mixed, since $f^{!}=j^{!} P^{!}=j^{*} P^{!}$.

Remark 2.8.1. As a consequence of Lafforgue's theorem on the Langlands correspondence for function fields and a Ramanujan-Petersson type of result, one deduces that all complexes on any $\mathbb{F}_{q}$-algebraic stack is $\iota$-mixed, for any $\iota$. To see this, by Proposition 2.8 and Lemma $2.5(\mathrm{ii}, \mathrm{v})$, we reduce to the case of an irreducible lisse sheaf on a smooth (in particular, normal) $\mathbb{F}_{q}$-scheme. By [Deligne $1980,1.3 .6]$ we reduce to the case where the determinant of the lisse sheaf has finite order, and Lafforgue's result [Laumon 2002, 1.3] applies. In the following, when we want to emphasize the assumption of $\iota$-mixedness, we will still write $W_{m}\left(\mathscr{L}_{0}, \overline{\mathbb{Q}}_{\ell}\right)^{\prime \prime}$, although it equals the full category $W\left(\mathscr{X}_{0}, \overline{\mathbb{Q}}_{\ell}\right)$.

Next we show the stability of $\iota$-mixedness, first for a few operations on complexes on algebraic spaces, and then for all the six operations on stacks. Denote by $D_{\mathscr{L}_{0}}$ or just $D$ the dualizing functor $R \mathscr{H} o m\left(-, K_{\mathscr{L}_{0}}\right)$, where $K_{\mathscr{L}_{0}}$ is a dualizing complex on $\mathscr{L}_{0}$ [Laszlo and Olsson 2008b, §7].

2.9. Recall [Kiehl and Weissauer 2001, II 12.2] that, for $\mathbb{F}_{q}$-schemes and bounded complexes of sheaves on them, the operations $f_{*}, f_{!}, f^{*}, f^{!}, D$ and $-\otimes^{L}-$ all preserve $\iota$-mixedness. Since we are working with $\overline{\mathbb{Q}}_{\ell}$-coefficients, $\otimes^{L}=\otimes$.

Lemma 2.10. Let $f: X_{0} \rightarrow Y_{0}$ be a morphism of $\mathbb{F}_{q}$-algebraic spaces. Then the operations $-\otimes-, D_{X_{0}}, f_{*}$ and $f_{!}$all preserve $\iota$-mixedness, namely, they induce 
functors

$$
\begin{gathered}
-\otimes-: W_{m}^{-}\left(X_{0}, \overline{\mathbb{Q}}_{\ell}\right) \times W_{m}^{-}\left(X_{0}, \overline{\mathbb{Q}}_{\ell}\right) \longrightarrow W_{m}^{-}\left(X_{0}, \overline{\mathbb{Q}}_{\ell}\right), \\
D: W_{m}\left(X_{0}, \overline{\mathbb{Q}}_{\ell}\right) \longrightarrow W_{m}\left(X_{0}, \overline{\mathbb{Q}}_{\ell}\right)^{\mathrm{op}}, \\
f_{*}: W_{m}^{+}\left(X_{0}, \overline{\mathbb{Q}}_{\ell}\right) \longrightarrow W_{m}^{+}\left(Y_{0}, \overline{\mathbb{Q}}_{\ell}\right), \\
f_{!}: W_{m}^{-}\left(X_{0}, \overline{\mathbb{Q}}_{\ell}\right) \longrightarrow W_{m}^{-}\left(Y_{0}, \overline{\mathbb{Q}}_{\ell}\right) .
\end{gathered}
$$

Proof. We will reduce to the case of unbounded complexes on schemes, and then prove the scheme case. Let $P: X_{0}^{\prime} \rightarrow X_{0}$ be an étale presentation.

Reduction for $\otimes$. For objects $K_{0}$ and $L_{0}$ in $W_{m}^{-}\left(X_{0}, \overline{\mathbb{Q}}_{\ell}\right)$, we have that

$$
P^{*}\left(K_{0} \otimes L_{0}\right)=\left(P^{*} K_{0}\right) \otimes\left(P^{*} L_{0}\right),
$$

and the reduction follows from Proposition 2.8.

Reduction for $D$. For $K_{0} \in W_{m}\left(X_{0}, \overline{\mathbb{Q}}_{\ell}\right)$, we have $P^{*} D K_{0}=D P^{!} K_{0}$, so the reduction follows from Proposition 2.8.

Reduction for $f_{*}$ and $f_{!}$. By definition [Laszlo and Olsson 2008b, 9.1] we have $f_{*}=D f_{!} D$, so it suffices to prove the case for $f_{!}$. Let $K_{0} \in W_{m}^{-}\left(X_{0}, \overline{\mathbb{Q}}_{\ell}\right)$, and let $P^{\prime}: Y_{0}^{\prime} \rightarrow Y_{0}$ and $X_{0}^{\prime} \rightarrow X_{0} \times_{Y_{0}} Y_{0}^{\prime}$ be étale presentations:

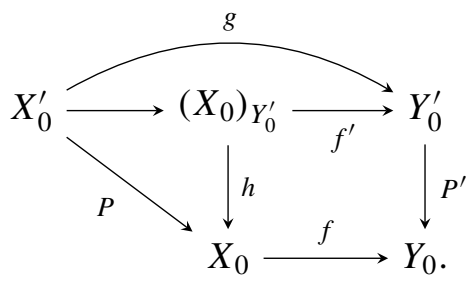

By smooth base change [Laszlo and Olsson 2008b, 12.1] we get $P^{*} f_{!} K_{0}=f_{!}^{\prime} h^{*} K_{0}$. Replacing $f$ by $f^{\prime}$ we can assume $Y_{0}$ is a scheme. Let $j: U_{0} \rightarrow X_{0}$ be an open dense subscheme [Knutson 1971, II 6.7], with complement $i: Z_{0} \rightarrow X_{0}$. Applying $f$ ! to the exact triangle

$$
j_{!} j^{*} K_{0} \longrightarrow K_{0} \longrightarrow i_{*} i^{*} K_{0} \longrightarrow
$$

we get

$$
(f j)_{!} j^{*} K_{0} \longrightarrow f_{!} K_{0} \longrightarrow(f i)_{!} i^{*} K_{0} \longrightarrow
$$

By Lemma 2.5 (iii) and noetherian induction, we can replace $X_{0}$ by $U_{0}$, and reduce to the case where $f$ is a morphism between schemes.

This finishes the reduction to the case of unbounded complexes on schemes, and now we prove this case.

For the Verdier dual $D_{X_{0}}$, since the dualizing complex $K_{X_{0}}$ has finite quasiinjective dimension, for every $K_{0} \in W_{m}\left(X_{0}, \overline{\mathbb{Q}}_{\ell}\right)$ and every integer $i$, there exist 
integers $a$ and $b$ such that

$$
\mathscr{H}^{i}\left(D_{X_{0}} K_{0}\right) \simeq \mathscr{H}^{i}\left(D_{X_{0}} \tau_{[a, b]} K_{0}\right),
$$

and by 2.9 , we see that $D_{X_{0}} K_{0}$ is $\iota$-mixed.

Next we prove the case of $\otimes$. For $K_{0}$ and $L_{0} \in W_{m}^{-}\left(X_{0}, \overline{\mathbb{Q}}_{\ell}\right)$, we have

$$
\mathscr{H}^{r}\left(K_{0} \otimes L_{0}\right)=\bigoplus_{i+j=r} \mathscr{H}^{i}\left(K_{0}\right) \otimes \mathscr{H}^{j}\left(L_{0}\right)
$$

The result follows from 2.9.

Finally we prove the case of $f_{*}$ and $f$ !. Let $K_{0} \in W_{m}^{+}\left(X_{0}, \overline{\mathbb{Q}}_{\ell}\right)$. Then we have the spectral sequence

$$
E_{2}^{i j}=R^{i} f_{*}\left(\mathscr{H}^{j} K_{0}\right) \Longrightarrow R^{i+j} f_{*} K_{0},
$$

and the result follows from 2.9 and Lemma 2.5 (i,ii). The case for $f_{!}=D f_{*} D$ also follows.

Finally we prove the main result of this section. This generalizes [Behrend 2003, 6.3.7].

Theorem 2.11. Let $f: \mathscr{X}_{0} \rightarrow \mathscr{Y}_{0}$ be a morphism of $\mathbb{F}_{q}$-algebraic stacks. Then the operations $f_{*}, f_{!}, f^{*}, f^{!}, D_{\mathscr{L}_{0}},-\otimes-$ and $R \mathscr{H} \operatorname{Oom}(-,-)$ all preserve $\iota-m i x e d n e s s$, namely, they induce functors

$$
\begin{gathered}
f_{*}: W_{m}^{+}\left(\mathscr{X}_{0}, \overline{\mathbb{Q}}_{\ell}\right) \longrightarrow W_{m}^{+}\left(\mathscr{Y}_{0}, \overline{\mathbb{Q}}_{\ell}\right), \quad f_{!}: W_{m}^{-}\left(\mathscr{X}_{0}, \overline{\mathbb{Q}}_{\ell}\right) \longrightarrow W_{m}^{-}\left(\mathscr{Y}_{0}, \overline{\mathbb{Q}}_{\ell}\right), \\
f^{*}: W_{m}\left(\mathscr{Y}_{0}, \overline{\mathbb{Q}}_{\ell}\right) \longrightarrow W_{m}\left(\mathscr{X}_{0}, \overline{\mathbb{Q}}_{\ell}\right), \quad f^{!}: W_{m}\left(\mathscr{Y}_{0}, \overline{\mathbb{Q}}_{\ell}\right) \longrightarrow W_{m}\left(\mathscr{Q}_{0}, \overline{\mathbb{Q}}_{\ell}\right), \\
R \mathscr{H o m}(-,-): W_{m}^{-}\left(\mathscr{Q}_{0}, \overline{\mathbb{Q}}_{\ell}\right)^{\mathrm{op}} \times W_{m}^{+}\left(\mathscr{X}_{0}, \overline{\mathbb{Q}}_{\ell}\right) \longrightarrow W_{m}^{+}\left(\mathscr{Q}_{0}, \overline{\mathbb{Q}}_{\ell}\right), \\
\otimes: W_{m}^{-}\left(\mathscr{X}_{0}, \overline{\mathbb{Q}}_{\ell}\right) \times W_{m}^{-}\left(\mathscr{X}_{0}, \overline{\mathbb{Q}}_{\ell}\right) \longrightarrow W_{m}^{-}\left(\mathscr{X}_{0}, \overline{\mathbb{Q}}_{\ell}\right), \\
D: W_{m}\left(\mathscr{X}_{0}, \overline{\mathbb{Q}}_{\ell}\right) \longrightarrow W_{m}\left(\mathscr{X}_{0}, \overline{\mathbb{Q}}_{\ell}\right)^{\mathrm{op}} .
\end{gathered}
$$

Proof. Recall from [Laszlo and Olsson 2008b, 9.1] that we have $f_{!}:=D f_{*} D$ and $f^{!}:=D f^{*} D$. By [ibid., 6.0.12, 7.3.1], for $K_{0} \in W^{-}\left(\mathscr{Q}_{0}, \overline{\mathbb{Q}}_{\ell}\right)$ and $L_{0} \in W^{+}\left(\mathscr{Q}_{0}, \overline{\mathbb{Q}}_{\ell}\right)$, we have

$$
\begin{array}{r}
D\left(K_{0} \otimes D L_{0}\right)=R \mathscr{H o m}\left(K_{0} \otimes D L_{0}, K_{\mathscr{L}_{0}}\right)=R \mathscr{H o m}\left(K_{0}, R \mathscr{H o m}\left(D L_{0}, K_{\mathscr{L}_{0}}\right)\right) \\
=R \mathscr{H o m}\left(K_{0}, D D L_{0}\right)=R \mathscr{H o m}\left(K_{0}, L_{0}\right) .
\end{array}
$$

Therefore it suffices to prove the result for $f_{*}, f^{*}, D$ and $-\otimes-$. The case of $f^{*}$ is proved in Lemma 2.5 (iv).

For $D$ : Let $P: X_{0} \rightarrow \mathscr{X}_{0}$ be a presentation. Since $P^{*} D=D P^{!}$, the result follows from Proposition 2.8 and Lemma 2.10 .

For $\otimes:$ Since we have $P^{*}\left(K_{0} \otimes L_{0}\right)=P^{*} K_{0} \otimes P^{*} L_{0}$, the result follows from Proposition 2.8 and Lemma 2.10. 
For $f_{*}$ and $f_{!}$: We will start with $f_{!}$, in order to use smooth base change to reduce to the case when $\mathscr{Y}_{0}$ is a scheme, and then turn to $f_{*}$ in order to use cohomological descent.

Let $K_{0} \in W_{m}^{-}\left(\mathscr{Q}_{0}, \overline{\mathbb{Q}}_{\ell}\right)$, and let $P: Y_{0} \rightarrow \mathscr{Y}_{0}$ be a presentation and the following diagram be 2-Cartesian:

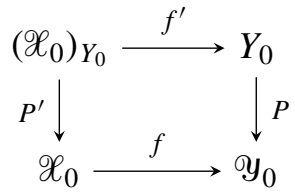

We have [ibid., 12.1] that $P^{*} f_{!} K_{0}=f_{!}^{\prime} P^{*} K_{0}$, so by 2.8 we can assume $\mathscr{Y}_{0}=Y_{0}$ is a scheme.

Now we switch to $f_{*}$, where $f: \mathscr{Q}_{0} \rightarrow Y_{0}$, and $K_{0} \in W_{m}^{+}\left(\mathscr{Q}_{0}, \overline{\mathbb{Q}}_{\ell}\right)$. Let $X_{0} \rightarrow \mathscr{X}_{0}$ be a presentation. Then it gives a strictly simplicial smooth hypercover $X_{0, \bullet}$ of $\mathscr{X}_{0}$ :

$$
X_{0, n}:=\underbrace{X_{0} \times \mathscr{Q}_{0} \cdots \times \mathscr{Q}_{0} X_{0}}_{n+1 \text { factors }}
$$

where each $X_{0, n}$ is an $\mathbb{F}_{q}$-algebraic space of finite type. Let $f_{n}: X_{0, n} \rightarrow Y_{0}$ be the restriction of $f$ to $X_{0, n}$. Then we have the spectral sequence [Laszlo and Olsson 2008b, 10.0.9]

$$
E_{1}^{i j}=R^{j} f_{i *}\left(\left.K_{0}\right|_{X_{0, i}}\right) \Longrightarrow R^{i+j} f_{*} K_{0} .
$$

Since $f_{i}$ 's are morphisms of algebraic spaces, the result follows from Lemma 2.10 and Lemma 2.5 (i, ii).

Remark 2.12. In fact, we can take the dualizing complex $K_{\mathscr{L}_{0}}$ to be mixed, and results in this section hold (and can be proved verbatim) for mixed complexes. In particular, mixedness is preserved by the six operations and the Verdier dualizing functor for stacks (if we take a mixed dualizing complex).

\section{Stratifiable complexes}

In this section, we use the same notations and hypotheses in 2.1. For the purpose of this article, it suffices to take $S$ to be Spec $k$ for an algebraically closed field $k$ of characteristic not equal to $\ell$, but we want to work in the general setting (namely, that of any scheme that satisfies (LO)) for future applications; for instance, when proving the generic base change. Let $\mathscr{X}, \mathscr{Y}, \ldots$ be $S$-algebraic stacks of finite type. By "sheaves" we mean "lisse-étale sheaves". "Locally constant constructible" is abbreviated as "lcc". A stratification $\mathscr{Y}$ of an $S$-algebraic stack $\mathscr{X}$ is a finite set of disjoint locally closed substacks that cover $\mathscr{X}$. If $\mathscr{F}$ is a lcc $\left(\Lambda_{n}\right) \mathscr{x}$-module, a decomposition series of $\mathscr{F}$ is a filtration by lcc $\Lambda_{\mathscr{L}}$-subsheaves, such that the successive 
quotients are simple $\Lambda_{\mathscr{X}}$-modules. Note that the filtration is always finite, and the simple successive quotients, which are $\left(\Lambda_{0}\right)_{\mathscr{X}}$-modules, are independent (up to order) of the decomposition series chosen. They are called the Jordan-Hölder components of $\mathscr{F}$.

Definition 3.1. (i) A complex $K=\left(K_{n}\right)_{n} \in \mathscr{D}_{c}(\mathscr{A})$ is said to be stratifiable if there exists a pair $(\mathscr{Y}, \mathscr{L})$, where $\mathscr{Y}$ is a stratification of $\mathscr{X}$ and $\mathscr{L}$ is a function that assigns to every stratum $U \in \mathscr{S}$ a finite set $\mathscr{L}(\mathcal{U})$ of isomorphism classes of simple (that is, irreducible) lcc $\Lambda_{0}$-modules on $\mathcal{U}_{\text {lis-ét, }}$ such that for each pair $(i, n)$ of integers the restriction of the sheaf $\mathscr{H}^{i}\left(K_{n}\right) \in \operatorname{Mod}_{c}\left(\mathscr{X}_{\text {lis-ét }}, \Lambda_{n}\right)$ to each stratum $U \in \mathscr{S}$ is lcc, with Jordan-Hölder components (as a $\Lambda_{U}$-module) contained in $\mathscr{L}(U)$. We say that the pair $(\mathscr{L}, \mathscr{L})$ trivializes $K$ (or $K$ is $(\mathscr{L}, \mathscr{L})$-stratifiable), and denote the full subcategory of $(\mathscr{Y}, \mathscr{L})$-stratifiable complexes by $\mathscr{D}_{\mathscr{S}, \mathscr{L}}(\mathscr{A})$. The full subcategory of stratifiable complexes in $\mathscr{D}_{c}(\mathscr{A})$ is denoted by $\mathscr{D}_{c}^{\text {stra }}(\mathscr{A})$.

(ii) Let $D_{c}^{\text {stra }}(\mathscr{X}, \Lambda)$ be the essential image of $\mathscr{D}_{c}^{\text {stra }}(\mathscr{A})$ in $D_{c}(\mathscr{X}, \Lambda)$, and we call the objects of $D_{c}^{\text {stra }}(\mathscr{Q}, \Lambda)$ stratifiable complexes of sheaves.

(iii) Let $E_{\lambda}$ be a finite extension of $\mathbb{Q}_{\ell}$ with ring of integers $\mathbb{O}_{\lambda}$. Then the definition above applies to $\Lambda=\mathrm{O}_{\lambda}$. Let $D_{c}^{\text {stra }}\left(\mathscr{X}, E_{\lambda}\right)$ be the essential image of $D_{c}^{\text {stra }}\left(\mathscr{X}, \mathscr{O}_{\lambda}\right)$ in $D_{c}\left(\mathscr{X}, E_{\lambda}\right)$. Finally we define

$$
D_{c}^{\text {stra }}\left(\mathscr{X}, \overline{\mathbb{Q}}_{\ell}\right)=2-\operatorname{colim}_{E_{\lambda}} D_{c}^{\text {stra }}\left(\mathscr{X}, E_{\lambda}\right) .
$$

Remark 3.1.1. (i) This notion is due to Beilinson, Bernstein and Deligne [1982], and Behrend [2003] used it to define his derived category for stacks. Many results in this section are borrowed from [Behrend 2003], but reformulated and reproved in terms of the derived categories defined in [Laszlo and Olsson 2008b].

(ii) Let $\mathscr{F}$ be a $\Lambda_{n}$-sheaf trivialized by a pair $(\mathscr{Y}, \mathscr{L})$, and let $\mathscr{G}$ be a subquotient sheaf of $\mathscr{F}$. Then $\mathscr{G}$ is not necessarily trivialized by $(\mathscr{S}, \mathscr{L})$. But if $\mathscr{G}$ is lcc on each stratum in $\mathscr{Y}$, then it is necessarily trivialized by $(\mathscr{L}, \mathscr{L})$.

3.2. We say that the pair $\left(\mathscr{Y}^{\prime}, \mathscr{L}^{\prime}\right)$ refines the pair $(\mathscr{Y}, \mathscr{L})$, if $\mathscr{Y}^{\prime}$ refines $\mathscr{Y}$, and for every $\mathscr{V} \in \mathscr{S}^{\prime}, \quad U \in \mathscr{S}$ and $L \in \mathscr{L}(\mathcal{U})$, such that $\mathscr{V} \subset \mathcal{U}$, the restriction $\left.L\right|_{\mathscr{V}}$ is trivialized by $\mathscr{L}^{\prime}(\mathscr{V})$. Given a pair $(\mathscr{Y}, \mathscr{L})$ and a refined stratification $\mathscr{S}^{\prime}$ of $\mathscr{Y}$, there is a canonical way to define $\mathscr{L}^{\prime}$ such that $\left(\mathscr{Y}^{\prime}, \mathscr{L}^{\prime}\right)$ refines $(\mathscr{L}, \mathscr{L})$ : for every $\mathscr{V} \in \mathscr{Y}^{\prime}$, we take $\mathscr{L}^{\prime}(\mathscr{V})$ to be the set of isomorphism classes of Jordan-Hölder components of the lcc sheaves $\left.L\right|_{v}$ for $L \in \mathscr{L}(U)$, where $U$ ranges over all strata in $\mathscr{Y}$ that contains $\mathscr{V}$. It is clear that the set of all pairs $(\mathscr{Y}, \mathscr{L})$ form a filtered direct system.

A pair $(\mathscr{Y}, \mathscr{L})$ is said to be tensor closed if for every $u \in \mathscr{S}$ and $L, M \in \mathscr{L}(\mathcal{U})$, the sheaf tensor product $L \otimes_{\Lambda_{0}} M$ has Jordan-Hölder components in $\mathscr{L}(U)$.

For a pair $(\mathscr{Y}, \mathscr{L})$, a tensor closed hull of this pair is a tensor closed refinement.

Lemma 3.3. Every pair $(\mathscr{Y}, \mathscr{L})$ can be refined to a tensor closed pair $\left(\mathscr{Y}^{\prime}, \mathscr{L}^{\prime}\right)$. 
Proof. First we show that for a lcc sheaf of sets $\mathscr{F}$ on $\mathscr{X}_{\text {lis-ét, }}$, there exists a finite étale morphism $f: \mathscr{Y} \rightarrow \mathscr{X}$ of algebraic $S$-stacks such that $f^{-1} \mathscr{F}$ is constant. Consider the total space [F्F] of the sheaf $\mathscr{F}$. Precisely, this is the category fibered in groupoids over (Aff $/ S$ ) with the underlying category described as follows. Its objects are triples $\left(U \in \operatorname{obj}(\operatorname{Aff} / S), u \in\right.$ obj $\left.\mathscr{X}(U), s \in\left(u^{-1} \mathscr{F}\right)(U)\right)$, and morphisms from $(U, u, s)$ to $(V, v, t)$ are pairs $(f: U \rightarrow V, \alpha: v f \Rightarrow u)$ such that $t$ is mapped to $s$ under the identification $\alpha: f^{-1} v^{-1} \mathscr{F} \cong u^{-1} \mathscr{F}$. The map $(U, u, s) \mapsto(U, u)$ gives a map $g:[\mathscr{F}] \rightarrow \mathscr{X}$, which is representable finite étale (because it is so locally). The pullback sheaf $g^{-1 \mathscr{F}}$ on [F्F] has a global section, so the total space breaks up into two parts, one part being mapped isomorphically onto the base [F्F]. By induction on the degree of $g$ we are done.

Next we show that for a fixed representable finite étale morphism $\mathscr{Y} \rightarrow \mathscr{X}$, there are only finitely many isomorphism classes of simple lcc $\Lambda_{0}$-sheaves on $\mathscr{L}$ that become constant when pulled back to $\mathscr{Y}$. We can assume that both $\mathscr{X}$ and $\mathscr{Y}$ are connected. By the following Lemma 3.3.1, we reduce to the case where $\mathscr{y} \rightarrow \mathscr{X}$ is Galois with group $G$, for some finite group $G$. Then simple lcc $\Lambda_{0}$-sheaves on $\mathscr{L}$ that become constant on $\mathscr{Y}$ correspond to simple left $\Lambda_{0}[G]$-modules, which are cyclic and hence isomorphic to $\Lambda_{0}[G] / I$ for left maximal ideals $I$ of $\Lambda_{0}[G]$. There are only finitely many such ideals since $\Lambda_{0}[G]$ is a finite set.

Also note that a lcc subsheaf of a constant constructible sheaf on a connected stack is also constant. Let $L$ be a lcc subsheaf on $\mathscr{X}$ of the constant sheaf associated to a finite set $M$. Consider their total spaces. We have an inclusion of substacks $i:[L] \hookrightarrow \bigsqcup_{m \in M} \mathscr{X}_{m}$, where each part $\mathscr{X}_{m}$ is identified with $\mathscr{X}$. Then $i^{-1}\left(\mathscr{X}_{m}\right) \rightarrow \mathscr{X}_{m}$ is finite étale, and is the inclusion of a substack, hence is either an equivalence or the inclusion of the empty substack, since $\mathscr{L}$ is connected. It is clear that $L$ is also constant, associated to the subset of those $m \in M$ for which $i^{-1}\left(\mathscr{L}_{m}\right) \neq \varnothing$.

Finally we prove the lemma. Refining $\mathscr{S}$ if necessary, we assume all strata are connected stacks. For each stratum $u \in \mathscr{Y}$, let $\mathscr{Y} \rightarrow \mathcal{U}$ be a representable finite étale morphism, such that all sheaves in $\mathscr{L}(U)$ become constant on $\mathscr{Y}$. Then define $\mathscr{L}^{\prime}(U)$ to be the set of isomorphism classes of simple lcc $\Lambda_{0}$-sheaves on $U_{\text {lis-ét }}$ which become constant on $\mathscr{Y}$. For any $L$ and $M \in \mathscr{L}^{\prime}(U)$, since all lcc subsheaves of $L \otimes_{\Lambda_{0}} M$ are constant on $\mathscr{Y}$, we see that $L \otimes_{\Lambda_{0}} M$ has Jordan-Hölder components in $\mathscr{L}^{\prime}(U)$ and hence $\left(\mathscr{Y}, \mathscr{L}^{\prime}\right)$ is a tensor closed refinement of $(\mathscr{Y}, \mathscr{L})$.

Lemma 3.3.1. Let $\mathrm{y} \rightarrow \mathscr{X}$ be a representable finite étale morphism between connected $S$-algebraic stacks. Then there exists a morphism $\mathscr{L} \rightarrow \mathscr{Y}$, such that $\mathscr{L}$ is Galois over $\mathscr{X}$, that is, it is a $G$-torsor for some finite group $G$.

Proof. Assume $\mathscr{X}$ is nonempty, and take a geometric point $\bar{x} \rightarrow \mathscr{X}$. Let $\mathscr{C}$ be the category FÉt $(\mathscr{C})$ of representable finite étale morphisms to $\mathscr{X}$, and let

$$
F: \mathscr{C} \rightarrow \text { FSet }
$$


be the fiber functor to the category of finite sets, namely $F(\mathscr{y})=\operatorname{Hom}_{\mathscr{L}}(\bar{x}, \mathcal{Y})$. Note that this Hom, which is a priori a category, is a finite set, since $\mathscr{Y} \rightarrow \mathscr{X}$ is representable and finite. Then one can verify that $(\mathscr{C}, F: \mathscr{C} \rightarrow$ FSet) satisfies the axioms of Galois formalism in [Grothendieck and Raynaud 1971, Exp. V, 4], and use the consequence g) on p. 121 in (loc. cit.) For the reader's convenience, we follow Olsson's suggestion and explain the proof briefly. Basically, we will verify certain axioms of (G1)-(G6), and deduce the conclusion as in (loc. cit.).

First note that $\mathscr{b}$, which is a priori a 2-category, is a 1-category. This is because for any 2-commutative diagram

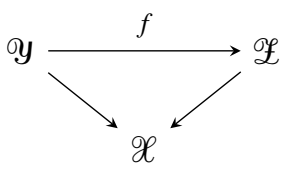

where $\mathscr{Y}$ and $\mathscr{E}$ are in $\mathscr{C}$, the morphism $f$ is also representable (and finite étale), so $\operatorname{Hom}_{\mathscr{L}}(\mathscr{Y}, \mathscr{L})$ is discrete. By definition, the functor $F$ preserves fiber-products, and $F(\mathscr{X})$ is a one-point set.

Let $f: \mathscr{Y} \rightarrow \mathscr{L}$ be a morphism in $\mathscr{C}$, then it is finite étale. So if the degree of $f$ is 1 , then $f$ is an isomorphism. This implies that the functor $F$ is conservative, that is, $f$ is an isomorphism if $F(f)$ is. In particular, $f$ is a monomorphism if and only if $F(f)$ is. This is because $f$ is a monomorphism if and only if $p_{1}: \mathscr{Y} \times \mathscr{E}$ y $\rightarrow$ y is an isomorphism, and $F$ preserves fiber-products.

Since $f: \mathscr{Y} \rightarrow \mathscr{L}$ is finite étale, its image stack $\mathscr{Y}^{\prime} \subset \mathscr{L}$ is both open and closed, hence $\mathscr{Y}^{\prime} \rightarrow \mathscr{L}$ is a monomorphism that is an isomorphism onto a direct summand of $\mathscr{L}$ (that is, $\mathscr{L}=\mathscr{Y}^{\prime} \amalg \mathscr{y}^{\prime \prime}$ for some other open and closed substack $\mathscr{Y}^{\prime \prime} \subset \mathscr{Z}$ ). Also, since $\mathscr{Y} \rightarrow \mathcal{Y}^{\prime}$ is epic and finite étale, it is strictly epic, that is, for every $\mathscr{L} \in \mathscr{C}$, the diagram

$$
\operatorname{Hom}\left(\mathscr{Y}^{\prime}, \mathscr{L}\right) \rightarrow \operatorname{Hom}(\mathscr{Y}, \mathscr{L}) \rightrightarrows \operatorname{Hom}\left(\mathscr{Y} \times y^{\prime} \text { Y }, \mathscr{L}\right)
$$

is an equalizer.

Every object $\mathscr{Y}$ in $\mathscr{C}$ is artinian: for a chain of monomorphisms

$$
\cdots \rightarrow \mathscr{Y}_{n} \rightarrow \cdots \rightarrow \mathscr{Y}_{2} \rightarrow \mathscr{Y}_{1} \rightarrow \mathscr{y}
$$

we get a chain of injections

$$
\cdots \rightarrow F\left(\mathscr{Y}_{n}\right) \rightarrow \cdots \rightarrow F\left(\mathscr{Y}_{1}\right) \rightarrow F(\mathscr{Y}),
$$

which is stable since $F(y)$ is a finite set, and so the first chain is also stable since $F$ is conservative.

Since $F$ is left exact and every object in $\mathscr{C}$ is artinian, by [Grothendieck 1960, 3.1] the functor $F$ is strictly pro-representable, that is, there exists a projective system $P=\left\{P_{i} ; i \in I\right\}$ of objects in $\mathscr{C}$ indexed by a filtered partially ordered set $I$, 
with epic transition morphisms $\varphi_{i j}: P_{j} \rightarrow P_{i}(i \leq j)$, such that there is a natural isomorphism of functors

$$
F \stackrel{\sim}{\longrightarrow} \operatorname{Hom}(P,-):=\operatorname{colim}_{I} \operatorname{Hom}\left(P_{i},-\right) .
$$

Let $\psi_{i}: P \rightarrow P_{i}$ be the canonical projection in the category Pro( $\left.\mathscr{C}\right)$ of pro-objects of $\mathscr{C}$. We may assume that every epimorphism $P_{j} \rightarrow \mathscr{L}$ in $\mathscr{C}$ is isomorphic to $P_{j} \stackrel{\varphi_{i j}}{\longrightarrow} P_{i}$ for some $i \leq j$. This is because one can add $P_{j} \rightarrow \mathscr{L}$ into the projective system $P$ without changing the functor it represents. Also one can show that the $P_{i}$ 's are connected [Grothendieck 1960], and morphisms in $\mathscr{C}$ between connected stacks are strictly epic.

Given $\mathscr{Y} \in \mathscr{C}$, now we show that there exists an object $\mathscr{L} \rightarrow \mathscr{X}$ that is Galois and factors through $Y$. Since $F(y)$ is a finite set, there exists an index $j \in I$ such that all maps $P \rightarrow$ Y factors through $P \stackrel{\psi_{j}}{\longrightarrow} P_{j}$. This means that the canonical map

$$
P \rightarrow \mathscr{Y}^{J}:=\underbrace{\mathscr{y} \times \mathscr{X} \cdots \times \mathscr{Y} \mathscr{y}}_{\# J \text { factors }}, \quad \text { where } J:=F(\mathscr{Y})=\operatorname{Hom}_{\operatorname{Pro}(\mathscr{C})}(P, \mathscr{Y})
$$

factors as

$$
P \stackrel{\psi_{j}}{\longrightarrow} P_{j} \stackrel{A}{\longrightarrow} \mathscr{Y}^{J}
$$

Let $P_{j} \rightarrow P_{i} \stackrel{B}{\rightarrow} y^{J}$ be the factorization of $A$ into a composition of an epimorphism and a monomorphism $B$. We claim that $P_{i}$ is Galois over $\mathscr{X}$.

Since $F\left(P_{i}\right)$ is a finite set, there exists an index $k \in I$ such that all maps $P \rightarrow P_{i}$ factors through $P \stackrel{\psi_{k}}{\longrightarrow} P_{k}$. Fix any $v: P_{k} \rightarrow P_{i}$. To show $P_{i}$ is Galois, it suffices to show that $\operatorname{Aut}\left(P_{i}\right)$ acts on $F\left(P_{i}\right)=\operatorname{Hom}\left(P_{k}, P_{i}\right)$ transitively, that is, there exists a $\sigma \in \operatorname{Aut}\left(P_{i}\right)$ making the triangle commute:

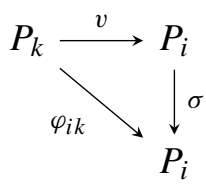

For every $u \in J=\operatorname{Hom}\left(P_{i}, \mathscr{Y}\right)$, we have $u \circ v \in \operatorname{Hom}\left(P_{k}\right.$, $\left.\mathscr{Y}\right)$, so there exists a $u^{\prime} \in \operatorname{Hom}\left(P_{i}\right.$, Yy) making the diagram commute:

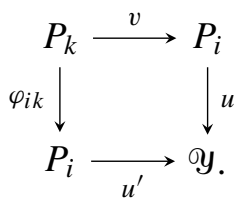

Since $v$ is epic, the function $u \mapsto u^{\prime}: J \rightarrow J$ is injective, hence a bijection. Let $\alpha: y^{J} \rightarrow y^{J}$ be the isomorphism induced by the map $u \mapsto u^{\prime}$. Then the diagram 


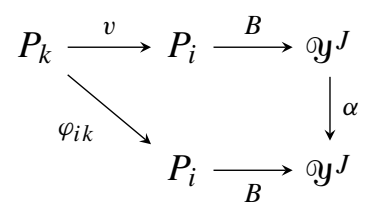

commutes. By the uniqueness of the factorization of the map $P_{k} \rightarrow y^{J}$ into the composition of an epimorphism and a monomorphism, there exists a $\sigma \in \operatorname{Aut}\left(P_{i}\right)$ such that $\sigma \circ v=\varphi_{i k}$. This finishes the proof.

We give some basic properties of stratifiable complexes.

Lemma 3.4. (i) $\mathscr{D}_{c}^{\text {stra }}(\mathscr{A})$ (respectively $D_{c}^{\text {stra }}(\mathscr{X}, \Lambda)$ ) is a triangulated subcategory of $\mathscr{D}_{c}(\mathscr{A})$ (respectively $D_{c}(\mathscr{L}, \Lambda)$ ) with the induced standard $t$-structure.

(ii) If $f: \mathscr{X} \rightarrow \mathscr{Y}$ is an $S$-morphism, then $f^{*}: \mathscr{D}_{c}(\mathscr{A}(\mathscr{Y})) \rightarrow \mathscr{D}_{c}(\mathscr{A}(\mathscr{X})$ ) (respectively $\left.f^{*}: D_{c}(\mathscr{Y}, \Lambda) \rightarrow D_{c}(\mathscr{X}, \Lambda)\right)$ preserves stratifiability.

(iii) If $\mathscr{Y}$ is a stratification of $\mathscr{X}$, then $K \in \mathscr{D}_{c}(\mathscr{A}(\mathscr{X}))$ is stratifiable if and only if $\left.K\right|_{V}$ is stratifiable for every $V \in \mathscr{Y}$.

(iv) Let $P: X \rightarrow \mathscr{X}$ be a presentation, and let $K=\left(K_{n}\right)_{n} \in \mathscr{D}_{c}(\mathscr{A}(\mathscr{X}))$. Then $K$ is stratifiable if and only if $P^{*} K$ is stratifiable.

(v) $D_{c}^{\text {stra }}(\mathscr{X}, \Lambda)$ contains $D_{c}^{b}(\mathscr{X}, \Lambda)$, and the heart of $D_{c}^{\text {stra }}(\mathscr{X}, \Lambda)$ is the same as that of $D_{c}(\mathscr{X}, \Lambda)$ in Remark 2.2.1 (i).

(vi) Let $K \in \mathscr{D}_{c}(\mathscr{A})$ be a normalized complex [Laszlo and Olsson 2008b, 3.0.8]. Then $K$ is trivialized by a pair $(\mathscr{Y}, \mathscr{L})$ if and only if $K_{0}$ is trivialized by this pair. (vii) Let $K \in \mathscr{D}_{c}^{\text {stra }}(\mathscr{A})$. Then its Tate twist $K(1)$ is also stratifiable.

Proof. (i) To show $\mathscr{D}_{c}^{\text {stra }}(\mathscr{A})$ is a triangulated subcategory, it suffices to show [Deligne 1977, p. 271] that for every exact triangle $K^{\prime} \rightarrow K \rightarrow K^{\prime \prime} \rightarrow K^{\prime}[1]$ in $\mathscr{D}_{c}(\mathscr{A})$, if $K^{\prime}$ and $K^{\prime \prime}$ are stratifiable, so also is $K$.

Using refinement we may assume that $K^{\prime}$ and $K^{\prime \prime}$ are trivialized by the same pair $(\mathscr{L}, \mathscr{L})$. Consider the cohomology sequence of this exact triangle at level $n$, restricted to a stratum $u \in \mathscr{Y}$. By Olsson [2007, 9.1], to show that a sheaf is lcc on $U$, one can pass to a presentation $U$ of the stack $U$. Then by Milne $[2008,20.3]$ and the five-lemma, we see that the $\mathscr{H}^{i}\left(K_{n}\right)$ 's are lcc on $\mathcal{U}$, with Jordan-Hölder components contained in $\mathscr{L}(\mathscr{U})$. Therefore $\mathscr{D}_{c}^{\text {stra }}(\mathscr{A})$ (and hence $D_{c}^{\text {stra }}(\mathscr{X}, \Lambda)$ ) is a triangulated subcategory.

The $t$-structure is inherited by $\mathscr{D}_{c}^{\text {stra }}(\mathscr{A})$ (and hence by $D_{c}^{\text {stra }}(\mathscr{X}, \Lambda)$ ) because, if $K \in \mathscr{D}_{c}(\mathscr{A})$ is stratifiable, so also are its truncations $\tau_{\leq r} K$ and $\tau_{\geq r} K$.

(ii) The functor $f^{*}$ is exact on the level of sheaves, and takes a lcc sheaf to a lcc sheaf. If $\left(K_{n}\right)_{n} \in \mathscr{D}_{c}(\mathscr{A}(\mathscr{Y}))$ is trivialized by $(\mathscr{Y}, \mathscr{L})$, then $\left(f^{*} K_{n}\right)_{n}$ is trivialized by $\left(f^{*} \mathscr{Y}, f^{*} \mathscr{L}\right)$, where $f^{*} \mathscr{Y}=\left\{f^{-1}(V) \mid V \in \mathscr{Y}\right\}$ and $\left(f^{*} \mathscr{L}\right)\left(f^{-1}(V)\right)$ is the set of 
isomorphism classes of Jordan-Hölder components of $f^{*} L, L \in \mathscr{L}(V)$. The case of $D_{c}(-, \Lambda)$ follows easily.

(iii) The "only if" part follows from (ii). The "if" part is clear: if $\left(\mathscr{Y}_{V}, \mathscr{L}_{V}\right)$ is a pair on $V$ that trivializes $\left(\left.K_{n}\right|_{V}\right)_{n}$, then the pair $\left(\mathscr{Y}_{\mathscr{L}}, \mathscr{L}\right)$ on $\mathscr{X}$, where $\mathscr{S}_{\mathscr{X}}=\cup \mathscr{S}_{V}$ and $\mathscr{L}=\left\{\mathscr{L}_{V}\right\}_{V \in \mathscr{S}}$, trivializes $\left(K_{n}\right)_{n}$.

(iv) The "only if" part follows from (ii). For the "if" part, assume $P^{*} K$ is trivialized by a pair $\left(\mathscr{S}_{X}, \mathscr{L}_{X}\right)$ on $X$. Let $U \in \mathscr{S}_{X}$ be an open stratum, and let $V \subset \mathscr{X}$ be the image of $U$ [Laumon and Moret-Bailly 2000, 3.7]. Recall that for every $T$ in Aff $/ S, V(T)$ is the full subcategory of $\mathscr{L}(T)$ consisting of objects $x$ that are locally in the essential image of $U(T)$, that is, such that there exists an étale surjection $T^{\prime} \rightarrow T$ in Aff $/ S$ and $u^{\prime} \in U\left(T^{\prime}\right)$, such that the image of $u^{\prime}$ in $\mathscr{X}\left(T^{\prime}\right)$ and $\left.x\right|_{T^{\prime}}$ are isomorphic. Then $V$ is an open substack of $\mathscr{X}$ (hence also an algebraic stack) and $\left.P\right|_{U}: U \rightarrow V$ is a presentation. Replacing $P: X \rightarrow \mathscr{X}$ by $\left.P\right|_{U}: U \rightarrow V$ and using noetherian induction and (iii), we may assume $\mathscr{Y}_{X}=\{X\}$.

It follows from a theorem of Gabber [Illusie et al. 2008] that $P_{*}$ takes a bounded complex to a bounded complex. In fact, using base change by $P$, we may assume that $P: Y \rightarrow X$ is a morphism from an $S$-algebraic space $Y$ to an $S$-scheme $X$. Let $j: U \rightarrow Y$ be an open dense subscheme of $Y$ with complement $i: Z \rightarrow Y$. For a bounded complex $L$ of $\Lambda_{n}$-sheaves on $Y$, we have the exact triangle

$$
(P i)_{*} i ! L \longrightarrow P_{*} L \longrightarrow(P j)_{*} j^{*} L \longrightarrow .
$$

Gabber's theorem implies that $(P j)_{*} j^{*} L$ is bounded, since $P j: U \rightarrow X$ is a morphism between schemes. Note that the dualizing functor preserves boundedness, so does $i^{!}=D_{Z} i^{*} D_{Y}$, and therefore we may assume that $(P i)_{*} i^{!} L$ is bounded by noetherian induction. It follows that $P_{*} L$ is bounded.

Now take a pair $(\mathscr{Y}, \mathscr{L})$ on $\mathscr{X}$ that trivializes all $P_{*} L$ 's, for $L \in \mathscr{L}_{X}$; this is possible since each $P_{*} L$ is bounded and $\mathscr{L}_{X}$ is a finite set. We claim that $K$ is trivialized by $(\mathscr{Y}, \mathscr{L})$.

For each sheaf $\mathscr{F}$ on $\mathscr{X}$, the natural map $\mathscr{F} \rightarrow R^{0} P_{*} P^{*} \mathscr{F}$ is injective. This follows from the sheaf axioms for the lisse-lisse topology, and the fact that the lisse-étale topos and the lisse-lisse topos are the same. Explicitly, to verify the injectivity on $X_{U} \rightarrow U$, for any $u \in \mathscr{L}(U)$, since the question is étale local on $U$, one can assume $P: X_{U} \rightarrow U$ has a section $s: U \rightarrow X_{U}$. Then the composition $\mathscr{F}_{U} \rightarrow R^{0} P_{*} P^{*} \mathscr{F}_{U} \rightarrow R^{0} P_{*} R^{0} s_{*} s^{*} P^{*} \mathscr{F}_{U}=\mathscr{F}_{U}$ of the two adjunctions is the adjunction for $P \circ s=\mathrm{id}$, so the composite is an isomorphism, and the first map is injective.

We take $\mathscr{F}_{F}$ to be the cohomology sheaves $\mathscr{H}^{i}\left(K_{n}\right)$. Since $P^{*} \mathcal{H}^{i}\left(K_{n}\right)$ is an iterated extension of sheaves in $\mathscr{L}_{X}$, we see that $P_{*} P^{*} \mathscr{H}^{i}\left(K_{n}\right)$, and in particular $R^{0} P_{*} P^{*} \mathscr{H}^{i}\left(K_{n}\right)$, are trivialized by $(\mathscr{Y}, \mathscr{L})$ by (i). Since $\mathscr{H}^{i}\left(K_{n}\right)$ is lcc [Olsson 
2007, 9.1], by Remark 3.1.1 (ii) we see that $\mathscr{H}^{i}\left(K_{n}\right)$ (hence $K$ ) is trivialized by $(\mathscr{Y}, \mathscr{L})$.

(v) By part (i) and Remark 2.2.1 (i) it is enough to show that all adic systems $M=\left(M_{n}\right)_{n} \in \mathscr{A}$ are stratifiable. By (iv) we may assume $\mathscr{X}=X$ is an $S$-scheme. Since $X$ is noetherian, there exists a stratification [Illusie 1977, VI, 1.2.6] of $X$ such that $M$ is lisse on each stratum. By (iii) we may assume $M$ is lisse on $X$.

Let $\mathscr{L}$ be the set of isomorphism classes of Jordan-Hölder components of the $\Lambda_{0}$-sheaf $M_{0}$. We claim that $\mathscr{L}$ trivializes $M_{n}$ for all $n$. Suppose it trivializes $M_{n-1}$ for some $n \geq 1$. Consider the sub- $\Lambda_{n}$-modules $\lambda M_{n} \subset M_{n}\left[\lambda^{n}\right] \subset M_{n}$, where $M_{n}\left[\lambda^{n}\right]$ is the kernel of the map $\lambda^{n}: M_{n} \rightarrow M_{n}$. Since $M$ is adic, we have exact sequences of $\Lambda_{X}$-modules

$$
\begin{aligned}
& 0 \longrightarrow M_{n} \longrightarrow M_{n} \longrightarrow M_{0} \longrightarrow 0, \\
& 0 \longrightarrow M_{n}\left[\lambda^{n}\right] \longrightarrow M_{n} \longrightarrow \lambda^{n} M_{n} \longrightarrow 0, \\
& 0 \longrightarrow \lambda^{n} M_{n} \longrightarrow M_{n} \longrightarrow M_{n-1} \longrightarrow 0 .
\end{aligned}
$$

The natural surjection $M_{n} / \lambda M_{n} \rightarrow M_{n} / M_{n}\left[\lambda^{n}\right]$ implies that $\mathscr{L}$ trivializes $\lambda^{n} M_{n}$, and therefore it also trivializes $M_{n}$. By induction on $n$ we are done.

Since $D_{c}^{b} \subset D_{c}^{\text {stra }} \subset D_{c}$, and $D_{c}^{b}$ and $D_{c}$ have the same heart, it is clear that $D_{c}^{\text {stra }}$ has the same heart as them.

(vi) Applying $-\otimes_{\Lambda_{n}}^{L} K_{n}$ to the following exact sequence, viewed as an exact triangle in $\mathscr{D}\left(\mathscr{X}, \Lambda_{n}\right)$

$$
0 \longrightarrow \Lambda_{n-1} \stackrel{1 \mapsto \lambda}{\longrightarrow} \Lambda_{n} \longrightarrow \Lambda_{0} \longrightarrow 0,
$$

we get an exact triangle by Laszlo and Olsson [2008b, 3.0.10]

$$
K_{n-1} \longrightarrow K_{n} \longrightarrow K_{0} \longrightarrow \text {. }
$$

By induction on $n$ and Remark 3.4.1 below, we see that $K$ is trivialized by $(\mathscr{Y}, \mathscr{L})$ if $K_{0}$ is.

(vii) Let $K=\left(K_{n}\right)_{n}$. Recall that the Tate twist $K(1)$ is defined to be the system $\left(K_{n}(1)\right)_{n}$, where $K_{n}(1)=K_{n} \otimes_{\Lambda_{n}}^{L} \Lambda_{n}(1)$. Note that the sheaf $\Lambda_{n}(1)$ is a flat $\Lambda_{n^{-}}$ module: to show that $-\otimes_{\Lambda_{n}} \Lambda_{n}$ (1) preserves injections, one can pass to stalks at geometric points, over which we have a trivialization $\Lambda_{n} \simeq \Lambda_{n}(1)$.

Suppose $K$ is $(\mathscr{L}, \mathscr{L})$-stratifiable. Using the isomorphism

$$
\mathscr{H}^{i}\left(K_{n}\right) \otimes_{\Lambda_{n}} \Lambda_{n}(1)=\mathscr{H}^{i}\left(K_{n} \otimes_{\Lambda_{n}}^{L} \Lambda_{n}(1)\right),
$$

it suffices to show the existence of a pair $\left(\mathscr{Y}, \mathscr{L}^{\prime}\right)$ such that for each $U \in \mathscr{Y}$, the Jordan-Hölder components of the lcc sheaves $L \otimes_{\Lambda_{n}} \Lambda_{n}(1)$ lie in $\mathscr{L}^{\prime}(\mathcal{U})$, for all 
$L \in \mathscr{L}(U)$. Since $L$ is a $\Lambda_{0}$-module, we have

$$
\begin{aligned}
L \otimes_{\Lambda_{n}} \Lambda_{n}(1)=\left(L \otimes_{\Lambda_{n}} \Lambda_{0}\right) \otimes_{\Lambda_{n}} \Lambda_{n}(1)=L \otimes_{\Lambda_{n}}\left(\Lambda_{0} \otimes_{\Lambda_{n}} \Lambda_{n}(1)\right) & \\
& =L \otimes_{\Lambda_{n}} \Lambda_{0}(1)=L \otimes_{\Lambda_{0}} \Lambda_{0}(1),
\end{aligned}
$$

and we can take $\mathscr{L}^{\prime}(\mathcal{U})$ to be a tensor closed hull of $\left\{\Lambda_{0}(1), L \in \mathscr{L}(\mathcal{U})\right\}$.

Remark 3.4.1. In fact the proof of Lemma 3.4 (i) shows that $\mathscr{D} \mathscr{Y}, \mathscr{L}(\mathscr{A})$ is a triangulated subcategory with induced standard $t$-structure, for each fixed pair $(\mathscr{Y}, \mathscr{L})$. Let $D_{\mathscr{S}, \mathscr{L}}(\mathscr{X}, \Lambda)$ be the essential image of $\mathscr{D} \mathscr{S}, \mathscr{L}(\mathscr{A})$ in $D_{c}(\mathscr{X}, \Lambda)$, and this is also a triangulated subcategory with induced standard $t$-structure.

Also if $E_{r}^{i j} \Longrightarrow E^{n}$ is a spectral sequence in the category $\mathscr{A}(\mathscr{X})$, and the $E_{r}^{i j}$ 's are trivialized by $(\mathscr{Y}, \mathscr{L})$ for all $i, j$, then all the $E^{n}$ 's are trivialized by $(\mathscr{Y}, \mathscr{L})$.

We denote by $D_{c}^{\dagger}$,stra $(\mathscr{X}, \Lambda)$, for $\dagger= \pm, b$, the full subcategory of $\dagger$-bounded stratifiable complexes, using the induced $t$-structure.

The following is a key result for showing the stability of stratifiability under the six operations later. Recall that $M \mapsto \widehat{M}=L \pi^{*} R \pi_{*} M$ is the normalization functor, where $\pi: \mathscr{L}^{\mathbb{N}} \rightarrow \mathscr{X}$ is the morphism of topoi in [Laszlo and Olsson 2008b, 2.1], mentioned in 2.3 .

Proposition 3.5. For a pair $(\mathscr{S}, \mathscr{L})$ on $\mathscr{X}$, if $M \in \mathscr{D}_{\mathscr{S}, \mathscr{L}}(\mathscr{A})$, then $\widehat{M} \in \mathscr{D}_{\mathscr{S}, \mathscr{L}}(\mathscr{A})$, too. In particular, if $K \in D_{c}(\mathscr{Q}, \Lambda)$, then $K$ is stratifiable if and only if its normalization $\widehat{K} \in \mathscr{D}_{c}(\mathscr{A})$ is stratifiable.

Proof. First, we will reduce to the case where $M$ is essentially bounded (that is, $\mathscr{H}^{i} M$ is AR-null for $|i| \gg 0$ ). Let $P: X \rightarrow \mathscr{X}$ be a presentation. The $\ell$ cohomological dimension of $X_{\text {ét }}$ is finite, by the assumption (LO) on $S$. Therefore, by Laszlo and Olsson [2008b, 2.1.i], the normalization functor for $X$ has finite cohomological dimension, and the same is true for $\mathscr{X}$ since $P^{*} \widehat{M}=\widehat{P^{*} M}$, by [ibid., 2.2.1, 3.0.11]. This implies that for each integer $i$, there exist integers $a$ and $b$ with $a \leq b$, such that $\mathscr{H}^{i}(\widehat{M})=\mathscr{H}^{i}\left(\widehat{\tau_{[a, b]} M}\right)$. Since $\tau_{[a, b]} M$ is also trivialized by $(\mathscr{Y}, \mathscr{L})$, we may assume $M \in \mathscr{D}_{\mathscr{S}, \mathscr{L}}^{b}(\mathscr{A}(\mathscr{L}))$.

Since $\widehat{M}$ is normalized, by Lemma 3.4 (vi), it suffices to show that $(\widehat{M})_{0}$ is trivialized by $(\mathscr{Y}, \mathscr{L})$. Using projection formula and the flat resolution of $\Lambda_{0}$

$$
0 \longrightarrow \Lambda \stackrel{\lambda}{\longrightarrow} \Lambda \stackrel{\varepsilon}{\longrightarrow} \Lambda_{0} \longrightarrow 0,
$$

we have [ibid., p.176]

$$
(\widehat{M})_{0}=\Lambda_{0} \otimes_{\Lambda}^{L} R \pi_{*} M=R \pi_{*}\left(\pi^{*} \Lambda_{0} \otimes_{\Lambda}^{L} M\right),
$$

where $\pi^{*} \Lambda_{0}$ is the constant projective system defined by $\Lambda_{0}$. Let $C \in \mathscr{D}(\mathscr{A})$ be the complex of projective systems $\pi^{*} \Lambda_{0} \otimes_{\Lambda_{\bullet}}^{L} M$; it is a $\lambda$-complex, and we have $C_{n}=\Lambda_{0} \otimes_{\Lambda_{n}}^{L} M_{n} \in \mathscr{D}_{c}\left(\mathscr{X}, \Lambda_{0}\right)$. 
Recall [Illusie 1977, V, 3.2.3] that a projective system $\left(K_{n}\right)_{n}$ ringed by $\Lambda_{\bullet}$ in an abelian category is AR-adic if and only if

- it satisfies the condition (MLAR) [Illusie 1977, V, 2.1.1], hence (ML), and denote by $\left(N_{n}\right)_{n}$ the projective system of the universal images of $\left(K_{n}\right)_{n}$;

- there exists an integer $k \geq 0$ such that the projective system $\left(L_{n}\right)_{n}:=\left(N_{n+k} \otimes \Lambda_{n}\right)_{n}$ is adic.

Moreover, $\left(K_{n}\right)_{n}$ is AR-isomorphic to $\left(L_{n}\right)_{n}$. Now for each $i$, the projective system $\mathscr{H}^{i}(C)$ is AR-adic Remark 2.2.1(i). Let $N^{i}=\left(N_{n}^{i}\right)_{n}$ be the projective system of the universal images of $\mathscr{H}^{i}(C)$, and choose an integer $k \geq 0$ such that the system $L^{i}=\left(L_{n}^{i}\right)_{n}=\left(N_{n+k}^{i} \otimes \Lambda_{n}\right)_{n}$ is adic. Since $N_{n+k}^{i} \subset \mathscr{H}^{i}\left(C_{n+k}\right)$ is annihilated by $\lambda$, we have $L_{n}^{i}=N_{n+k}^{i}$, and the transition morphism gives an isomorphism

$$
L_{n}^{i} \simeq L_{n}^{i} \otimes_{\Lambda_{n}} \Lambda_{n-1} \stackrel{\sim}{\longrightarrow} L_{n-1}^{i} .
$$

This means the projective system $L^{i}$ is the constant system $\pi^{*} L_{0}^{i}$. By Laszlo and Olsson [2008b, 2.2.2] we have $R \pi_{*} \mathscr{H}^{i}(C) \simeq R \pi_{*} L^{i}$, which is just $L_{0}^{i}$ by [ibid., 2.2.3].

The spectral sequence

$$
R^{j} \pi_{*} \mathscr{H}^{i}(C) \Longrightarrow \mathscr{H}^{i+j}\left((\widehat{M})_{0}\right)
$$

degenerates to isomorphisms $L_{0}^{i} \simeq \mathscr{H}^{i}\left((\widehat{M})_{0}\right)$, so we only need to show that $L_{0}^{i}$ is trivialized by $(\mathscr{Y}, \mathscr{L})$. Using the periodic $\Lambda_{n}$-flat resolution of $\Lambda_{0}$

$$
\cdots \longrightarrow \Lambda_{n} \stackrel{\lambda}{\longrightarrow} \Lambda_{n} \stackrel{\lambda^{n}}{\longrightarrow} \Lambda_{n} \stackrel{\lambda}{\longrightarrow} \Lambda_{n} \stackrel{\varepsilon}{\longrightarrow} \Lambda_{0} \longrightarrow 0,
$$

we see that $\Lambda_{0} \otimes_{\Lambda_{n}}^{L} \mathscr{H}^{j}\left(M_{n}\right)$ is represented by the complex

$$
\cdots \longrightarrow \mathscr{H}^{j}\left(M_{n}\right) \stackrel{\lambda^{n}}{\longrightarrow} \mathscr{H}^{j}\left(M_{n}\right) \stackrel{\lambda}{\longrightarrow} \mathscr{H}^{j}\left(M_{n}\right) \longrightarrow 0,
$$

so $\mathscr{H}^{i}\left(\Lambda_{0} \otimes_{\Lambda_{n}}^{L} \mathscr{H}^{j}\left(M_{n}\right)\right)$ are trivialized by $(\mathscr{Y}, \mathscr{L})$, for all $i, j$. Since $M$ is essentially bounded, we have the spectral sequence

$$
\mathscr{H}^{i}\left(\Lambda_{0} \otimes_{\Lambda_{n}}^{L} \mathscr{H}^{j}\left(M_{n}\right)\right) \Longrightarrow \mathscr{H}^{i+j}\left(C_{n}\right),
$$

from which we deduce (by Remark 3.4.1) that the $\mathscr{H}^{i}\left(C_{n}\right)$ 's are trivialized by $(\mathscr{Y}, \mathscr{L})$. The universal image $N_{n}^{i}$ is the image of $\mathscr{H}^{i}\left(C_{n+r}\right) \rightarrow \mathscr{H}^{i}\left(C_{n}\right)$ for some $r \gg 0$, therefore the $N_{n}^{i}$,s (and hence the $L_{n}^{i}$ 's) are trivialized by $(\mathscr{Y}, \mathscr{L})$.

For the second claim, let $K \in D_{c}(\mathscr{X}, \Lambda)$. Since $K$ is isomorphic to the image of $\widehat{K}$ under the localization $\mathscr{D}_{c}(\mathscr{A}) \rightarrow D_{c}(\mathscr{X}, \Lambda)$ [Laszlo and Olsson 2008b, 3.0.14], we see that $K$ is stratifiable if $\widehat{K}$ is. Conversely, if $K$ is stratifiable, which means that it is isomorphic to the image of some $M \in \mathscr{D}_{c}^{\text {stra }}(\mathscr{A})$, then $\widehat{K}=\widehat{M}$ is also stratifiable. 
3.5.1. For $K \in D_{c}(\mathscr{X}, \Lambda)$, we say that $K$ is $(\mathscr{Y}, \mathscr{L})$-stratifiable if $\widehat{K}$ is, and then Proposition 3.5 implies that $K \in D_{\mathscr{S}, \mathscr{L}}(\mathscr{X}, \Lambda)$ (see Remark 3.4.1) if and only if $K$ is $(\mathscr{Y}, \mathscr{L})$-stratifiable.

Corollary 3.6. (i) If $\mathscr{Y}$ is a stratification of $\mathscr{X}$, then $K \in D_{c}(\mathscr{X}, \Lambda)$ is stratifiable if and only if $\left.K\right|_{V}$ is stratifiable for every $V \in \mathscr{Y}$.

(ii) Let $K \in D_{c}(\mathscr{X}, \Lambda)$. Then $K$ is stratifiable if and only if its Tate twist $K(1)$ is.

(iii) Let $P: X \rightarrow \mathscr{X}$ be a presentation, and let $K \in D_{c}(\mathscr{X}, \Lambda)$. Then $K$ is stratifiable if and only if $P^{*} K$ (respectively $P^{!} K$ ) is stratifiable.

Proof. (i) The "only if" part follows from Lemma 3.4 (ii). For the "if" part, we first prove the following.

Lemma 3.6.1. For an S-algebraic stack $\mathscr{L}$ locally of finite type, let

$$
N \stackrel{u}{\longrightarrow} M \longrightarrow C \longrightarrow N[1]
$$

be an exact triangle in $\mathscr{D}_{c}(\mathscr{A})$, where $N$ is a normalized complex and $C$ is almost AR-null. Then the morphism $u$ is isomorphic to the natural map $\widehat{M} \rightarrow M$.

Proof. Consider the following diagram

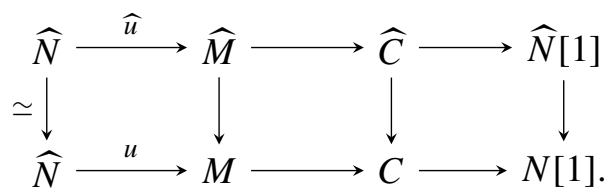

Since $C$ is almost AR-null, we have $\widehat{C}=0$ by Laszlo and Olsson [2008b, 2.2.2], and so $\widehat{u}$ is an isomorphism.

Now let $f: \mathscr{V} \rightarrow \mathscr{X}$ be a morphism of $S$-algebraic stacks, and let $M \in \mathscr{D}_{c}(\mathscr{A}(\mathscr{X}))$. We claim that $f^{*} \widehat{M} \simeq \widehat{f^{*} M}$. Applying $f^{*}$ to the exact triangle

$$
\widehat{M} \longrightarrow M \longrightarrow C \longrightarrow
$$

we get

$$
f^{*} \widehat{M} \longrightarrow f^{*} M \longrightarrow f^{*} C \longrightarrow .
$$

By Laszlo and Olsson [2008a, 4.3.2], $\widehat{M}_{n}=\operatorname{hocolim}_{N} \tau_{\leq N} \widehat{M}_{n}$, and $-\otimes_{\Lambda_{n}}^{L} \Lambda_{n-1}$ and $f^{*}$ preserve homotopy colimit because they preserve infinite direct sums. Now that $\tau_{\leq N} \widehat{M}_{n}$ and $\Lambda_{n-1}$ are bounded above complexes, we have

$$
f^{*}\left(\tau_{\leq N} \widehat{M}_{n} \otimes_{\Lambda_{n}}^{L} \Lambda_{n-1}\right) \simeq f^{*} \tau_{\leq N} \widehat{M}_{n} \otimes_{\Lambda_{n}}^{L} \Lambda_{n-1}
$$

(see the proof of [ibid., 4.5.3]). Hence applying $f^{*}$ to the isomorphism

$$
\widehat{M}_{n} \otimes_{\Lambda_{n}}^{L} \Lambda_{n-1} \longrightarrow \widehat{M}_{n-1}
$$


we get an isomorphism

$$
f^{*} \widehat{M}_{n} \otimes_{\Lambda_{n}}^{L} \Lambda_{n-1} \longrightarrow f^{*} \widehat{M}_{n-1}
$$

and by [ibid., 3.0.10], $f^{*} \widehat{M}$ is normalized. Also it is clear that $f^{*} C$ is AR-null. By Lemma 3.6.1 we have $f^{*} \widehat{M} \simeq \widehat{f^{*} M}$.

Therefore, the "if" part follows from Lemma 3.4 (iii) and Proposition 3.5, since $\left.\widehat{K}\right|_{V} \simeq \widehat{\left(\left.K\right|_{V}\right)}$

(ii) This follows from Lemma 3.4 (vii), since $\widehat{K}(1)=\widehat{K(1)}$.

(iii) For $P^{*} K$, the "only if" part follows from Lemma 3.4 (ii), and the "if" part follows from Lemma 3.4 (iv) and Proposition 3.5, since $P^{*} \widehat{K}=\widehat{\left(P^{*} K\right)}$ [ibid., 2.2.1, 3.0.11].

Since $P$ is smooth of relative dimension $d$, for some function $d: \pi_{0}(X) \rightarrow \mathbb{N}$, we have $P^{!} K \simeq P^{*} K(d)[2 d]$, so by (ii), $P^{*} K$ is stratifiable if and only if $P^{!} K$ is.

Before proving the main result of this section, we prove some special cases.

3.7. Let $f: X \rightarrow Y$ be a morphism of $S$-schemes. Then the $\Lambda_{n}$-dualizing complexes $K_{X, n}$ and $K_{Y, n}$ of $X$ and $Y$ respectively have finite quasi-injective dimensions, and are bounded by some integer independent of $n$. Together with the base change theorem for $f_{!}$, we see that there exists an integer $N>0$ depending only on $X, Y$ and $f$, such that for any integers $a, b$ and $n$ with $n \geq 0$ and any $M \in \mathscr{D}_{c}^{[a, b]}\left(X, \Lambda_{n}\right)$, we have $f_{*} M \in \mathscr{D}_{c}^{[a, b+N]}\left(Y, \Lambda_{n}\right)$. This implies that for each $n$, the functor (defined using $K$-injective resolutions, see [Spaltenstein 1988, 6.7])

$$
f_{*}: \mathscr{D}\left(X, \Lambda_{n}\right) \rightarrow \mathscr{D}\left(Y, \Lambda_{n}\right)
$$

restricts to

$$
f_{*}: \mathscr{D}_{c}\left(X, \Lambda_{n}\right) \rightarrow \mathscr{D}_{c}\left(Y, \Lambda_{n}\right)
$$

Moreover, for $M \in \mathscr{D}(\mathscr{A}(X))$ with constructible $\mathscr{H}^{j}\left(M_{n}\right)$ 's (for all $j$ and $n$ ) and for each $i \in \mathbb{Z}$, there exist integers $a<b$ such that

$$
R^{i} f_{*} M \simeq R^{i} f_{*} \tau_{[a, b]} M
$$

In particular, if $M$ is a $\lambda$-complex on $X$, then $R^{i} f_{*} M$ is AR-adic for each $i$, and hence $f_{*} M=\left(f_{*} M_{n}\right)_{n}$ is a $\lambda$-complex on $Y$.

This enables us to define

$$
f_{*}: D_{c}(X, \Lambda) \rightarrow D_{c}(Y, \Lambda)
$$

to be $K \mapsto Q f_{*} \widehat{K}$, where $Q: \mathscr{D}_{c}(\mathscr{A}(Y)) \rightarrow D_{c}(Y, \Lambda)$ is the localization functor. It agrees with the definition in [Laszlo and Olsson 2008b, 8] when restricted to 
$D_{c}^{+}(X, \Lambda)$, and for each $i \in \mathbb{Z}$ and $K \in D_{c}(X, \Lambda)$, there exist integers $a<b$ such that $R^{i} f_{*} K \simeq R^{i} f_{*} \tau_{[a, b]} K$.

Lemma 3.8. (i) If $f: X \rightarrow Y$ is a morphism of $S$-schemes, and $K \in D_{c}(X, \Lambda)$ is trivialized by $(\{X\}, \mathscr{L})$ for some $\mathscr{L}$, then $f_{*} K$ is stratifiable.

(ii) Let $\mathscr{X}$ be an $S$-algebraic stack that has a connected presentation (that is, there exists a presentation $P: X \rightarrow \mathscr{X}$ with $X$ a connected $S$-scheme). Let $K_{\mathscr{X}}$ and $K_{\mathscr{X}}^{\prime}$ be two $\Lambda$-dualizing complexes on $\mathscr{X}$, and let $D$ and $D^{\prime}$ be the two associated dualizing functors, respectively. Let $K \in D_{c}(\mathscr{X}, \Lambda)$. If $D K$ is trivialized by a pair $(\mathscr{Y}, \mathscr{L})$, where all strata in $\mathscr{Y}$ are connected, then $D^{\prime} K$ is trivialized by $\left(\mathscr{Y}, \mathscr{L}^{\prime}\right)$ for some other $\mathscr{L}^{\prime}$. In particular, for stacks with connected presentation, the property of the Verdier dual of $K$ being stratifiable is independent of the choice of the dualizing complex.

(iii) Let $\mathscr{Q}$ be an $S$-algebraic stack that has a connected presentation, and assume that the constant sheaf $\Lambda$ on $\mathscr{X}$ is a dualizing complex. If $K \in D_{c}(\mathscr{X}, \Lambda)$ is trivialized by a pair $(\{\mathscr{X}\}, \mathscr{L})$, then $D_{\mathscr{L}} K$ is trivialized by $\left(\{\mathscr{X}\}, \mathscr{L}^{\prime}\right)$ for some $\mathscr{L}^{\prime}$.

Proof. (i) Since $f_{*} K$ is the image of $f_{*} \widehat{K}$, it suffices to show that $f_{*} \widehat{K}$ is stratifiable. Since $f_{*} L$ is bounded for each $L \in \mathscr{L}$, there exists a pair $\left(\mathscr{S}_{Y}, \mathscr{L}_{Y}\right)$ on $Y$ that trivializes $f_{*} L$, for all $L \in \mathscr{L}$. We claim that this pair trivializes $R^{i} f_{*} \widehat{K}_{n}$, for each $i$ and $n$.

Since $R^{i} f_{*} \widehat{K}_{n}=R^{i} f_{*} \tau_{[a, b]} \widehat{K}_{n}$ for some $a<b$, and $\tau_{[a, b]} \widehat{K}_{n}$ is trivialized by $(\{X\}, \mathscr{L})$, we may assume $\widehat{K}_{n}$ is bounded. The claim then follows from the spectral sequence

$$
R^{p} f_{*} \mathscr{H}^{q}\left((\widehat{K})_{n}\right) \Longrightarrow R^{p+q} f_{*}\left((\widehat{K})_{n}\right)
$$

and Remark 3.4.1.

(ii) Recall that the dualizing complex $K_{\mathscr{L}}$ (respectively $K_{\mathscr{X}}^{\prime}$ ) is defined to be the image of a normalized complex $K_{\mathscr{X}, \bullet}\left(\right.$ respectively $K_{\mathscr{Q}, \bullet}^{\prime}$ ), where each $K_{\mathscr{Q}, n}$ (respectively $K_{\mathscr{Q}, n}^{\prime}$ ) is a $\Lambda_{n}$-dualizing complex. See [ibid., 7.2.3, 7.2.4].

Let $P: X \rightarrow \mathscr{X}$ be a presentation where $X$ is a connected scheme. Then we have $P^{*} R \mathscr{H o m}\left(K_{\mathscr{X}, n}, K_{\mathscr{X}, n}^{\prime}\right)=R \mathscr{H o m}\left(P^{*} K_{\mathscr{X}, n}, P^{*} K_{\mathscr{X}, n}^{\prime}\right)=R \mathscr{H o m}\left(P^{!} K_{\mathscr{X}, n}, P^{!} K_{\mathscr{X}, n}^{\prime}\right)$.

Since $P^{!} K_{\mathscr{X}, n}$ and $P^{!} K_{\mathscr{Q}, n}^{\prime}$ are $\Lambda_{n}$-dualizing complexes on $X$, by [Illusie 1977, Exp. I, 2], we see that $P^{*} R \mathscr{H} o m\left(K_{\mathscr{X}, n}, K_{\mathscr{Q}, n}^{\prime}\right)$ (and hence $R \mathscr{H} o m\left(K_{\mathscr{X}, n}, K_{\mathscr{Q}, n}^{\prime}\right)$ ) is cohomologically concentrated in one degree, therefore it is quasi-isomorphic to this nontrivial cohomology sheaf, once it has been appropriately shifted. So let $R \mathscr{H o m}\left(K_{\mathscr{L}, n}, K_{\mathscr{X}, n}^{\prime}\right) \simeq L_{n}\left[r_{n}\right]$ for some sheaf $L_{n}$ and integer $r_{n}$. Since $P^{*} L_{n}$ is invertible and hence lcc (see [Illusie 1977, p. 19]), the sheaf $L_{n}$ is lcc [Olsson 2007, 9.1]. 
For every stratum $u \in \mathscr{T}$, let $\mathscr{L}_{0}(u)$ be the union of $\mathscr{L}(U)$ and the set of isomorphism classes of the Jordan-Hölder components of the lcc sheaf $L_{0} \mid u$. Since all strata in $\mathscr{Y}$ are connected, there exists a tensor closed hull of $\left(\mathscr{Y}, \mathscr{L}_{0}\right)$ of the form $\left(\mathscr{Y}, \mathscr{L}^{\prime}\right)$, that is, they have the same stratification $\mathscr{Y}$.

By Laszlo and Olsson [2008b, 4.0.8], the system

$$
\left(L_{n}\left[r_{n}\right]\right)_{n}=R \mathscr{H o m}\left(\left(K_{\mathscr{X}, n}\right)_{n},\left(K_{\mathscr{Q}, n}^{\prime}\right)_{n}\right)
$$

is normalized, so by Lemma 3.6.1, $\widehat{D^{\prime} K_{\mathscr{X}}}=\left(L_{n}\left[r_{n}\right]\right)_{n}$, and by Lemma 3.4 (vi), it is trivialized by $\left(\mathscr{Y}, \mathscr{L}^{\prime}\right)$. Since $D K$ is trivialized by $\left(\mathscr{Y}, \mathscr{L}^{\prime}\right)$, so also is $D^{\prime} K$, because $\widehat{D^{\prime} K} \simeq \widehat{D K} \otimes^{L} \widehat{D^{\prime} K_{\mathscr{X}}}$.

(iii) The assumption implies in particular that $\mathscr{X}$ is connected, so by (ii), the question is independent of the choice of the dualizing complex. By definition, $\widehat{K}$ is trivialized by $(\{\mathscr{L}\}, \mathscr{L})$, so are truncations of $\widehat{K}$. The essential image of $R \mathscr{H o m}\left(\widehat{K}, \Lambda_{\bullet}\right)$ in $D_{c}(\mathscr{X}, \Lambda)$ is $D K$, so by 3.5.1 it suffices to show that $R \mathscr{H}$ om $\left(\widehat{K}, \Lambda_{\bullet}\right) \in \mathscr{D}_{\{\mathscr{L}\}, \mathscr{L}^{\prime}}(\mathscr{A})$ for some $\mathscr{L}^{\prime}$.

Since $\mathscr{X}$ is quasi-compact, each $\Lambda_{n}$-dualizing complex is of finite quasi-injective dimension, so for each integer $i$, there exist integers $a$ and $b$ such that

$$
\mathscr{H}^{i} R \mathscr{H o m}\left(\widehat{K}_{n}, \Lambda_{n}\right)=\mathscr{H}^{i} R \mathscr{H} \operatorname{Hom}\left(\tau_{[a, b]} \widehat{K}_{n}, \Lambda_{n}\right) .
$$

Using truncation triangles, we may further replace $\tau_{[a, b]} \widehat{K}_{n}$ by the cohomology sheaves $\mathscr{H}^{j} \widehat{K}_{n}$, and hence by their Jordan-Hölder components. Therefore, it suffices to find an $\mathscr{L}^{\prime}$ that trivializes $\mathscr{H}^{i} R \mathscr{H} o m\left(L, \Lambda_{0}\right)$, for all $i \in \mathbb{Z}$ and $L \in \mathscr{L}$. Note that $R \mathscr{H o m}\left(L, \Lambda_{0}\right)=\operatorname{Hom}\left(L, \Lambda_{0}\right)=L^{\vee}$ is a simple $\Lambda_{0}$-sheaf, so one can take $\mathscr{L}^{\prime}=\left\{L^{\vee} \mid L \in \mathscr{L}\right\}$.

Remark 3.8.1. For any $S$-algebraic stack $\mathscr{X}$, the Verdier dual of a complex $K$ in $D_{c}(\mathscr{X}, \Lambda)$ being stratifiable or not is independent of the choice of the dualizing complex. Let $K_{\mathscr{X}}$ and $K_{\mathscr{L}}^{\prime}$ be two dualizing complexes on $\mathscr{X}$, defining dualizing functors $D$ and $D^{\prime}$, respectively. Let $P: X \rightarrow \mathscr{X}$ be a presentation, let $K_{X}=P^{!} K_{\mathscr{L}}$ and let $K_{X}^{\prime}=P^{!} K_{\mathscr{x}}^{\prime}$, defining dualizing functors $D_{X}$ and $D_{X}^{\prime}$ on $X$, respectively. Suppose $D K$ is stratifiable. To show $D^{\prime} K$ is also stratifiable, by Corollary 3.6 (iii) it suffices to show $P^{!} D^{\prime} K=D_{X}^{\prime} P^{*} K$ is stratifiable. Since $D_{X} P^{*} K=P^{!} D K$ is stratifiable by assumption, we may assume $\mathscr{X}=X$ is a scheme. Since $X$ is noetherian, it has finitely many connected components, each of which is both open and closed. Then the result follows from Corollary 3.6 (i) and Lemma 3.8 (ii).

Next we prove the main result of this section.

Theorem 3.9. Let $f: \mathscr{X} \rightarrow$ y be a morphism of S-algebraic stacks. Then the operations $f_{*}, f_{!}, f^{*}, f^{!}, D_{\mathscr{L}},-\otimes^{L}-$ and $R \mathscr{H} \operatorname{Com}(-,-)$ all preserve stratifiability, 
namely, they induce functors

$$
\begin{gathered}
f_{*}: D_{c}^{+, \text {stra }}(\mathscr{X}, \Lambda) \longrightarrow D_{c}^{+, \text {stra }}(\mathscr{Y}, \Lambda), \quad f_{!}: D_{c}^{-, \text {stra }}(\mathscr{X}, \Lambda) \longrightarrow D_{c}^{-, \text {stra }}(\mathscr{Y}, \Lambda), \\
f^{*}: D_{c}^{\text {stra }}(\mathscr{Y}, \Lambda) \longrightarrow D_{c}^{\text {stra }}(\mathscr{X}, \Lambda), \quad f^{!}: D_{c}^{\text {stra }}(\mathscr{Y}, \Lambda) \longrightarrow D_{c}^{\text {stra }}(\mathscr{X}, \Lambda), \\
R \mathscr{H} \text { om }(-,-): D_{c}^{-, \text {stra }}(\mathscr{X}, \Lambda)^{\text {op }} \times D_{c}^{+, \text {stra }}(\mathscr{X}, \Lambda) \longrightarrow D_{c}^{+, \text {stra }}(\mathscr{X}, \Lambda), \\
\otimes^{L}: D_{c}^{-, \text {stra }}(\mathscr{X}, \Lambda) \times D_{c}^{-, \text {stra }}(\mathscr{X}, \Lambda) \longrightarrow D_{c}^{-, \text {stra }}(\mathscr{X}, \Lambda), \\
D: D_{c}^{\text {stra }}(\mathscr{X}, \Lambda) \longrightarrow D_{c}^{\text {stra }}(\mathscr{X}, \Lambda)^{\mathrm{op}} .
\end{gathered}
$$

Proof. We may assume all stacks involved are reduced.

We consider the Verdier dual functor $D$ first. Let $P: X \rightarrow \mathscr{X}$ be a presentation. Since $P^{*} D=D P^{!}$, by Corollary 3.6 (iii) we can assume $\mathscr{X}=X$ is a scheme. Let $K$ be a complex on $X$ trivialized by $(\mathscr{Y}, \mathscr{L})$. Refining if necessary, we may assume all strata in $\mathscr{S}$ are connected and regular. Let $j: U \rightarrow X$ be the immersion of an open stratum in $\mathscr{S}$ with complement $i: Z \rightarrow X$. Shrinking $U$ if necessary, we may assume there is a dimension function on $U$ [Riou 2007, Définition 2.1], hence by a result of Gabber [ibid., Théorème 0.2], the constant sheaf $\Lambda$ on $U$ is a dualizing complex. Consider the exact triangle

$$
i_{*} D_{Z}\left(\left.K\right|_{Z}\right) \longrightarrow D_{X} K \longrightarrow j_{*} D_{U}\left(\left.K\right|_{U}\right) \longrightarrow .
$$

By Lemma 3.8 (iii) we see that $D_{U}\left(\left.K\right|_{U}\right)$ is trivialized by $\left(\{U\}, \mathscr{L}^{\prime}\right)$ for some $\mathscr{L}^{\prime}$, so $j_{*} D_{U}\left(\left.K\right|_{U}\right)$ is stratifiable by Lemma $3.8(\mathrm{i})$. By noetherian induction we may assume $D_{Z}\left(\left.K\right|_{Z}\right)$ is stratifiable, and it is clear that $i_{*}$ preserves stratifiability. Therefore by Lemma $3.4(\mathrm{i}), D_{X} K$ is stratifiable.

The case of $f^{*}$ (and hence $f^{!}$) is proved in Lemma 3.4 (ii).

Next we prove the case of $\otimes^{L}$. For $i=1,2$, let $K_{i} \in D_{c}^{-}(\mathscr{X}, \Lambda)$, trivialized by $\left(\mathscr{Y}_{i}, \mathscr{L}_{i}\right)$. Let $(\mathscr{Y}, \mathscr{L})$ be a common tensor closed refinement (by Lemma 3.3) of $\left(\mathscr{S}_{i}, \mathscr{L}_{i}\right), i=1,2$. The total tensor product $K_{1} \otimes^{L} K_{2}$ is defined to be the image in $D_{c}(\mathscr{X}, \Lambda)$ of $\widehat{K}_{1} \otimes_{\Lambda}^{L} \widehat{K}_{2}$, which by Laszlo and Olsson [2008b, 3.0.10] is normalized, so it suffices to show (by Lemma 3.4 (vi)) that

$$
\widehat{K}_{1,0} \otimes_{\Lambda_{0}}^{L} \widehat{K}_{2,0}=\widehat{K}_{1,0} \otimes_{\Lambda_{0}} \widehat{K}_{2,0}
$$

is trivialized by $(\mathscr{L}, \mathscr{L})$. This follows from

$$
\mathscr{H}^{r}\left(\widehat{K}_{1,0} \otimes_{\Lambda_{0}} \widehat{K}_{2,0}\right)=\bigoplus_{i+j=r} \mathscr{H}^{i}\left(\widehat{K}_{1,0}\right) \otimes_{\Lambda_{0}} \mathscr{H}^{j}\left(\widehat{K}_{2,0}\right)
$$

and the assumption that $(\mathscr{Y}, \mathscr{L})$ is tensor closed.

The case of $R \mathscr{H o m}\left(K_{1}, K_{2}\right)=D\left(K_{1} \otimes^{L} D K_{2}\right)$ follows.

Finally we prove the case of $f_{*}$ and $f_{!}$. Let $f: \mathscr{L} \rightarrow \mathscr{Y}$ be a morphism of $S$ algebraic stacks, and let $K \in D_{\mathscr{S}, \mathscr{L}}^{-}(\mathscr{L}, \Lambda)$ for some pair $(\mathscr{Y}, \mathscr{L})$. We want to show 
$f_{!} K$ is stratifiable. Let $j: \mathscr{U} \rightarrow \mathscr{X}$ be the immersion of an open stratum in $\mathscr{Y}$, with complement $i: \mathscr{L} \rightarrow \mathscr{X}$. From the exact triangle

$$
(f j) ! j^{*} K \longrightarrow f_{!} K \longrightarrow(f i) ! i^{*} K \longrightarrow
$$

we see that it suffices to prove the claim for $f j$ and $f i$. By noetherian induction we can replace $\mathscr{X}$ by $\mathcal{U}$. By Corollary 3.6 (iii) and smooth base change [Laszlo and Olsson 2008b, 12.1], we can replace 9 by a presentation $Y$, and by Corollary 3.6 (i) and [ibid., 12.3] we can shrink $Y$ to an open subscheme. After these reductions, we assume that $y=Y$ is a regular irreducible affine $S$-scheme that has a dimension function on it, that $K$ is trivialized by $(\{\mathscr{X}\}, \mathscr{L})$, and that the relative inertia stack $\mathscr{I}_{f}:=\mathscr{X} \times_{\Delta, \mathscr{L} \times_{Y} \mathscr{X}, \Delta} \mathscr{X}$ is flat and has components over $\mathscr{X}$ [Behrend 2003, 5.1.14]. Therefore by [ibid., 5.1.13], $f$ factors as

$$
\mathscr{L} \stackrel{g}{\longrightarrow} \mathscr{L} \stackrel{h}{\longrightarrow} Y,
$$

where $g$ is gerbe-like and $h$ is representable (see [ibid., 5.1.3-5.1.6] for relevant notions). So we reduce to two cases: $f$ is representable, or $f$ is gerbe-like.

Case when $f$ is representable. By shrinking the $S$-algebraic space $\mathscr{X}$ we can assume $\mathscr{X}=X$ is a regular connected scheme that has a dimension function, so that the constant sheaf $\Lambda$ on $X$ is a dualizing complex. By Lemma 3.8 (iii) we see that $D K$ is trivialized by some $\left(\{X\}, \mathscr{L}^{\prime}\right)$, and by Lemma $3.8(\mathrm{i}), f_{*} D K$ is stratifiable. Therefore $f_{!} K=D f_{*} D K$ is also stratifiable.

Case when $f$ is gerbe-like. In this case $f$ is smooth [Behrend 2003, 5.1.5], hence étale locally on $Y$ it has a section. Replacing $Y$ by an étale cover, we may assume that $f$ is a neutral gerbe, so $f: B(G / Y) \rightarrow Y$ is the structural map, for some flat group space $G$ of finite type over $Y$ [Laumon and Moret-Bailly 2000, 3.21]. By [ibid., 5.1.1] and Corollary 3.6 (i) we may assume $G$ is a $Y$-group scheme. Next we reduce to the case when $G$ is smooth over $Y$.

By assumption $Y$ is integral. Let $k(Y)$ be the function field of $Y$ and $\overline{k(Y)}$ an algebraic closure. Then $G_{\overline{k(Y)}}$,red is smooth over $\overline{k(Y)}$, so there exists a finite extension $L$ over $k(Y)$ such that $G_{L \text {,red }}$ is smooth over $L$. Let $Y^{\prime}$ be the normalization of $Y$ in $L$, which is a scheme of finite type over $S$, and the natural map $Y^{\prime} \rightarrow Y$ is finite surjective. It factors through $Y^{\prime} \rightarrow Z \rightarrow Y$, where $Z$ is the normalization of $Y$ in the separable closure of $k(Y)$ in $L=k\left(Y^{\prime}\right)$. So $Z \rightarrow Y$ is generically étale, and $Y^{\prime} \rightarrow Z$ is purely inseparable, hence a universal homeomorphism, so $Y^{\prime}$ and $Z$ have equivalent étale sites. Replacing $Y^{\prime}$ by $Z$ and shrinking $Y$ we can assume $Y^{\prime} \rightarrow Y$ is finite étale. Replacing $Y$ by $Y^{\prime}$ (by Corollary 3.6 (ii)) we assume $G_{\text {red }}$ over $Y$ has smooth generic fiber, and by shrinking $Y$ we assume $G_{\text {red }}$ is smooth over $Y$.

$G_{\text {red }}$ is a subgroup scheme of $G$ [Grothendieck and Demazure 1970, Exposé $\left.\mathrm{VI}_{\mathrm{A}}, 0.2\right]$; we write $h: G_{\text {red }} \hookrightarrow G$ for the associated closed immersion. Then 
$B h: B\left(G_{\text {red }} / Y\right) \rightarrow B(G / Y)$ is faithful and hence representable. It is also radicial: consider the diagram where the square is 2-Cartesian

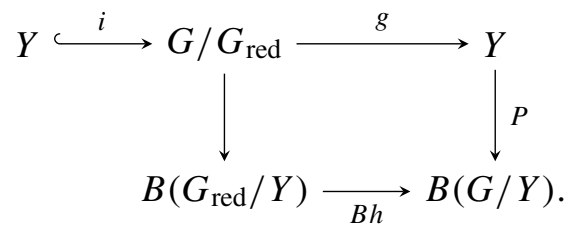

The map $i$ is a nilpotent closed embedding, so $g$ is radicial. Since $P$ is faithfully flat, $B h$ is also radicial. This shows that

$$
(B h)^{*}: D_{c}^{-}(B(G / Y), \Lambda) \rightarrow D_{c}^{-}\left(B\left(G_{\mathrm{red}} / Y\right), \Lambda\right)
$$

is an equivalence of categories. Replacing $G$ by $G_{\text {red }}$ we assume $G$ is smooth over $Y$, and hence $P: Y \rightarrow B(G / Y)$ is a connected presentation.

Let $d$ be the relative dimension of $G$ over $Y$. By assumption, the constant sheaf $\Lambda$ on $Y$ is a dualizing complex, and so $f^{!} \Lambda=\Lambda\langle-d\rangle$ (and hence the constant sheaf $\Lambda$ on $\mathscr{X}$ ) is a dualizing complex on $\mathscr{X}$. By Lemma 3.8 (iii), we see that $D K$ is trivialized by a pair of the form $\left(\{\mathscr{L}\}, \mathscr{L}^{\prime}\right)$. To show $f_{!} K$ is stratifiable is equivalent to showing that $D f_{!} K=f_{*} D K$ is stratifiable. So replacing $K$ by $D K$, it suffices to show that $f_{*} K$ is stratifiable, where $K \in D_{\{\mathscr{X}\}, \mathscr{L}}^{+}(\mathscr{X}, \Lambda)$ for some $\mathscr{L}$.

Consider the strictly simplicial smooth hypercover associated to the presentation $P: Y \rightarrow B(G / Y)$, and let $f_{i}: \prod_{i} G \rightarrow Y$ be the structural map. As in the proof of Lemma 3.8 (i), it suffices to show the existence of a pair $\left(\mathscr{S}_{Y}, \mathscr{L}_{Y}\right)$ on $Y$ that trivializes $R^{n} f_{*} L$, for all $L \in \mathscr{L}$ and $n \in \mathbb{Z}$. From the spectral sequence [Laszlo and Olsson 2008b, 10.0.9]

$$
E_{1}^{i j}=R^{j} f_{i *} f_{i}^{*} P^{*} L \Longrightarrow R^{i+j} f_{*} L,
$$

we see that it suffices for the pair $\left(\mathscr{S}_{Y}, \mathscr{L}_{Y}\right)$ to trivialize all the $E_{1}^{i j}$-terms. Assume $i \geq 1$. If we regard the map $f_{i}: \prod_{i} G \rightarrow Y$ as the product map

$$
\prod_{i} f_{1}: \prod_{i} G \rightarrow \prod_{i} Y
$$

where the products are fiber products over $Y$, then we can write $f_{i}^{*} P^{*} L$ as

$$
f_{1}^{*} P^{*} L \otimes_{\Lambda_{0}} \Lambda_{0} \otimes_{\Lambda_{0}} \cdots \otimes_{\Lambda_{0}} \Lambda_{0}
$$

Note that the scheme $Y$ satisfies the condition (LO). By Künneth formula [Laszlo and Olsson 2008b, 11.0.14] we have

$$
f_{i *} f_{i}^{*} P^{*} L=f_{1 *} f_{1}^{*} P^{*} L \otimes_{\Lambda_{0}} f_{1 *} \Lambda_{0} \otimes_{\Lambda_{0}} \cdots \otimes_{\Lambda_{0}} f_{1 *} \Lambda_{0} .
$$


Since $f_{1 *} f_{1}^{*} P^{*} L$ and $f_{1 *} \Lambda_{0}$ are bounded complexes (by a theorem of Gabber [Illusie et al. 2008]), there exists a tensor closed pair $\left(\mathscr{Y}_{Y}, \mathscr{L}_{Y}\right)$ that trivializes them, for all $L \in \mathscr{L}$. The proof is finished.

Consequently, the theorem also holds for $\overline{\mathbb{Q}}_{\ell}$-coefficients.

Finally we give a lemma which will be used in the next section. This will play the same role as [Behrend 2003, 6.3.16].

Lemma 3.10. Let $X$ be a connected variety over an algebraically closed field $k$ of characteristic not equal to $\ell$, and let $\mathscr{L}$ be a finite set of isomorphism classes of simple lcc $\Lambda_{0}$-sheaves on $X$. Then there exists an integer $d$ (depending only on $\mathscr{L})$ such that, for every lisse $\Lambda$-adic sheaf $\mathscr{F}$ on $X$ trivialized by $\mathscr{L}$, and for every integer $i$, we have

$$
\operatorname{dim}_{E} H_{c}^{i}\left(X, \mathscr{F} \otimes_{\Lambda} E\right) \leq d \cdot \operatorname{rank}_{E}\left(\mathscr{F} \otimes_{\Lambda} E\right),
$$

where $E$ is the fraction field of $\Lambda$.

Proof. Since $\mathscr{L}$ is finite and $0 \leq i \leq 2 \operatorname{dim} X$, there exists an integer $d>0$ such that $\operatorname{dim}_{\Lambda_{0}} H_{c}^{i}(X, L) \leq d \cdot \operatorname{rank}_{\Lambda_{0}} L$, for every $i$ and every $L \in \mathscr{L}$. For a short exact sequence of lcc $\Lambda_{0}$-sheaves

$$
0 \longrightarrow \mathscr{G}^{\prime} \longrightarrow \mathscr{G} \longrightarrow \mathscr{G}^{\prime \prime} \longrightarrow 0
$$

on $X$, the cohomological sequence

$$
\cdots \longrightarrow H_{c}^{i}\left(X, \mathscr{G}^{\prime}\right) \longrightarrow H_{c}^{i}(X, \mathscr{G}) \longrightarrow H_{c}^{i}\left(X, \mathscr{G}^{\prime \prime}\right) \longrightarrow \cdots
$$

implies that $\operatorname{dim}_{\Lambda_{0}} H_{c}^{i}(X, \mathscr{G}) \leq \operatorname{dim}_{\Lambda_{0}} H_{c}^{i}\left(X, \mathscr{G}^{\prime}\right)+\operatorname{dim}_{\Lambda_{0}} H_{c}^{i}\left(X, \mathscr{G}^{\prime \prime}\right)$. So it is clear that if $\mathscr{G}$ is trivialized by $\mathscr{L}$, then $\operatorname{dim}_{\Lambda_{0}} H_{c}^{i}(X, \mathscr{G}) \leq d \cdot \operatorname{rank}_{\Lambda_{0}} \mathscr{G}$, for every $i$.

Since we only consider $\mathscr{F}_{\mathcal{F}} \otimes_{\Lambda} E$, we may assume $\mathscr{F}_{F}=\left(\mathscr{F}_{n}\right)_{n}$ is flat, of some constant rank over $\Lambda$ (since $X$ is connected), and this $\Lambda$-rank is equal to

$$
\operatorname{rank}_{\Lambda_{0}} \mathscr{F}_{0}=\operatorname{rank}_{E}\left(\mathscr{F}_{\otimes_{\Lambda}} E\right) .
$$

Recall that $H_{c}^{i}(X, \mathscr{F})$ is a finitely generated $\Lambda$-module [Illusie $1977, \mathrm{VI}, 2.2 .2$ ], so by Nakayama's lemma the minimal number of generators of the module is at most $\operatorname{dim}_{\Lambda_{0}}\left(\Lambda_{0} \otimes_{\Lambda} H_{c}^{i}(X, \mathscr{F})\right)$. Similar to ordinary cohomology groups [Milne 2008, 19.2], we have an injection

$$
\Lambda_{0} \otimes_{\Lambda} H_{c}^{i}(X, \mathscr{F}) \hookrightarrow H_{c}^{i}\left(X, \mathscr{F}_{0}\right)
$$

of $\Lambda_{0}$-vector spaces. Therefore, $\operatorname{dim}_{E} H_{c}^{i}\left(X, \mathscr{F}_{\mathcal{F}} \otimes_{\Lambda} E\right)$ is less than or equal to the minimal number of generators of $H_{c}^{i}(X, \mathscr{F})$ over $\Lambda$, which is at most

$$
\begin{aligned}
\operatorname{dim}_{\Lambda_{0}}\left(\Lambda_{0} \otimes_{\Lambda} H_{c}^{i}(X, \mathscr{F})\right) & \leq \operatorname{dim}_{\Lambda_{0}} H_{c}^{i}\left(X, \mathscr{F}_{0}\right) \\
& \leq d \cdot \operatorname{rank}_{\Lambda_{0}} \mathscr{F}_{0}=d \cdot \operatorname{rank}_{E}\left(\mathscr{F}_{\mathbb{F}_{\Lambda}} E\right) .
\end{aligned}
$$




\section{Convergent complexes and finiteness}

We return to $\mathbb{F}_{q}$-algebraic stacks $\mathscr{X}_{0}, \mathscr{Y}_{0}, \ldots$ of finite type. A complex $K_{0}$ in $W\left(\mathscr{Q}_{0}, \overline{\mathbb{Q}}_{\ell}\right)$ is said to be stratifiable if $K$ on $\mathscr{X}$ is stratifiable, and we denote by $W^{\text {stra }}\left(\mathscr{Q}_{0}, \overline{\mathbb{Q}}_{\ell}\right)$ the full subcategory of such complexes. Note that if $K_{0}$ is a lisseétale complex, and it is stratifiable on $\mathscr{L}_{0}$, then it is geometrically stratifiable (that is, $K$ on $\mathscr{X}$ is stratifiable). In turns out that in order for the trace formula to hold, it suffices to make this weaker assumption of geometric stratifiability. So we will only discuss stratifiable Weil complexes. Again, by a sheaf we mean a Weil $\overline{\mathbb{Q}}_{\ell}$ sheaf.

Definition 4.1. (i) Let $K \in D_{c}\left(\overline{\mathbb{Q}}_{\ell}\right)$ and $\varphi: K \rightarrow K$ an endomorphism. The pair $(K, \varphi)$ is said to be an $\iota$-convergent complex (or just a convergent complex, since we fixed $\iota)$ if the complex series in two directions

$$
\sum_{n \in \mathbb{Z}} \sum_{H^{n}(K), H^{n}(\varphi)}|\alpha|^{s}
$$

is convergent, for every real number $s>0$. In this case let $\operatorname{Tr}(\varphi, K)$ be the absolutely convergent complex series

$$
\sum_{n}(-1)^{n} \iota \operatorname{Tr}\left(H^{n}(\varphi), H^{n}(K)\right)
$$

or its limit.

(ii) Let $K_{0} \in W^{-}\left(\mathscr{X}_{0}, \overline{\mathbb{Q}}_{\ell}\right)$. We call $K_{0}$ an l-convergent complex of sheaves (or just a convergent complex of sheaves), if for every integer $v \geq 1$ and every point $x \in \mathscr{L}_{0}\left(\mathbb{F}_{q^{v}}\right)$, the pair $\left(K_{\bar{x}}, F_{x}\right)$ is a convergent complex. In particular, all bounded complexes are convergent.

(iii) Let $K_{0} \in W^{-}\left(\mathscr{Q}_{0}, \overline{\mathbb{Q}}_{\ell}\right)$ be a convergent complex of sheaves. Define

$$
c_{v}\left(\mathscr{X}_{0}, K_{0}\right)=\sum_{x \in\left[\mathscr{X}_{0}\left(\mathbb{F}_{q^{v}}\right)\right]} \frac{1}{\# \operatorname{Aut}_{x} \mathbb{F}_{q^{v}}} \operatorname{Tr}\left(F_{x}, K_{\bar{x}}\right) \in \mathbb{C},
$$

and define the $L$-series of $K_{0}$ to be the formal power series

$$
L\left(\mathscr{C}_{0}, K_{0}, t\right)=\exp \left(\sum_{v \geq 1} c_{v}\left(\mathscr{C}_{0}, K_{0}\right) \frac{t^{v}}{v}\right) \in \mathbb{C}[[t]] .
$$

The zeta function $Z\left(\mathscr{X}_{0}, t\right)$ in Definition 1.2 is a special case of this definition as $Z\left(\mathscr{Q}_{0}, t\right)=L\left(\mathscr{X}_{0}, \overline{\mathbb{Q}}_{\ell}, t\right)$. It has rational coefficients.

Notation 4.1.1. We sometimes write $c_{v}\left(K_{0}\right)$ for $c_{v}\left(\mathscr{C}_{0}, K_{0}\right)$, if it is clear that $K_{0}$ is on $\mathscr{Q}_{0}$. We also write $c_{v}\left(\mathscr{Q}_{0}\right)$ for $c_{v}\left(\mathscr{X}_{0}, \overline{\mathbb{Q}}_{\ell}\right)$. 
Remark 4.1.2. (i) Behrend [2003, 6.2.3] defined convergent complexes with respect to arithmetic Frobenius elements, and our definition is for geometric Frobenius, and it is essentially the same as Behrend's definition, except we work with ı-mixed Weil complexes (which means all Weil complexes, by Remark 2.8.1) for an arbitrary isomorphism $\iota: \overline{\mathbb{Q}}_{\ell} \rightarrow \mathbb{C}$, while Behrend [2003] works with pure or mixed lisse-étale sheaves with integer weights. Also our definition is a little different from that in [Olsson 2008a]; the condition there is weaker.

(ii) Recall that Aut $x_{x}$ is defined to be the fiber over $x$ of the inertia stack $\Phi_{0} \rightarrow \mathscr{X}_{0}$. It is a group scheme of finite type [Laumon and Moret-Bailly 2000, 4.2] over $k(x)$, so $\operatorname{Aut}_{x} k(x)$ is a finite group.

(iii) If we have the following commutative diagram

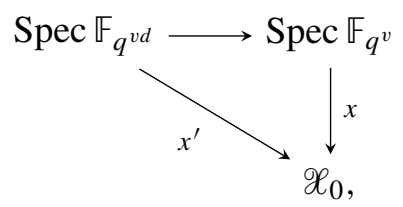

then $\left(K_{\bar{x}}, F_{x}\right)$ is convergent if and only if $\left(K_{\overline{x^{\prime}}}, F_{x^{\prime}}\right)$ is convergent, because we have $F_{x^{\prime}}=F_{x}^{d}$ and $s \mapsto s d: \mathbb{R}^{>0} \rightarrow \mathbb{R}^{>0}$ is a bijection. In particular, for a lisse-étale complex of sheaves, the property of being a convergent complex is independent of $q$ and the structural morphism $\mathscr{X}_{0} \rightarrow \operatorname{Spec} \mathbb{F}_{q}$. Also note that, for every integer $v \geq 1$, a complex $K_{0}$ on $\mathscr{X}_{0}$ is convergent if and only if $K_{0} \otimes \mathbb{F}_{q^{v}}$ on $\mathscr{X}_{0} \otimes \mathbb{F}_{q^{v}}$ is convergent.

We restate the main theorem in [Behrend 2003] using compactly supported cohomology as follows. It generalizes Theorem 1.1. We will prove it in this section and the next.

Theorem 4.2. Let $f: \mathscr{X}_{0} \rightarrow \mathscr{Y}_{0}$ be a morphism of $\mathbb{F}_{q}$-algebraic stacks, and let $K_{0} \in W_{m}^{-}$,stra $\left(\mathscr{L}_{0}, \overline{\mathbb{Q}}_{\ell}\right)$ be a convergent complex of sheaves. Then:

(i) (Finiteness) $f_{!} K_{0}$ is a convergent complex of sheaves on $\mathscr{Y}_{0}$.

(ii) (Trace formula) $c_{v}\left(\mathscr{C}_{0}, K_{0}\right)=c_{v}\left(\mathscr{Y}_{0}, f_{!} K_{0}\right)$ for every integer $v \geq 1$.

First we give a few lemmas.

Lemma 4.3. Let

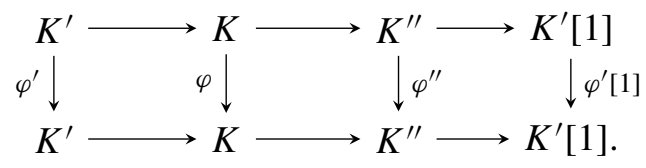

be an endomorphism of an exact triangle $K^{\prime} \rightarrow K \rightarrow K^{\prime \prime} \rightarrow K^{\prime}[1]$ in $D_{c}^{-}\left(\overline{\mathbb{Q}}_{\ell}\right)$. If any two of the three pairs $\left(K^{\prime}, \varphi^{\prime}\right),\left(K^{\prime \prime}, \varphi^{\prime \prime}\right)$ and $(K, \varphi)$ are convergent, then so is 
the third, and

$$
\operatorname{Tr}(\varphi, K)=\operatorname{Tr}\left(\varphi^{\prime}, K^{\prime}\right)+\operatorname{Tr}\left(\varphi^{\prime \prime}, K^{\prime \prime}\right) .
$$

Proof. By the rotation axiom we can assume $\left(K^{\prime}, \varphi^{\prime}\right)$ and $\left(K^{\prime \prime}, \varphi^{\prime \prime}\right)$ are convergent. We have the exact sequence

$$
\cdots \longrightarrow H^{n}\left(K^{\prime}\right) \longrightarrow H^{n}(K) \longrightarrow H^{n}\left(K^{\prime \prime}\right) \longrightarrow H^{n+1}\left(K^{\prime}\right) \longrightarrow \cdots .
$$

Since $H^{n}(K)$ is an extension of a subobject of $H^{n}\left(K^{\prime \prime}\right)$ by a quotient object of $H^{n}\left(K^{\prime}\right)$, we have

$$
\sum_{H^{n}(K), \varphi}|\alpha|^{s} \leq \sum_{H^{n}\left(K^{\prime}\right), \varphi^{\prime}}|\alpha|^{s}+\sum_{H^{n}\left(K^{\prime \prime}\right), \varphi^{\prime \prime}}|\alpha|^{s}
$$

for every real $s>0$, so $(K, \varphi)$ is convergent.

Since the series $\sum_{n \in \mathbb{Z}}(-1)^{n} \sum_{H^{n}(K), \varphi} \iota \alpha$ converges absolutely, we can change the order of summation, and the second assertion follows if we split the long exact sequence above into short exact sequences.

Corollary 4.4. If $K_{0}^{\prime} \rightarrow K_{0} \rightarrow K_{0}^{\prime \prime} \rightarrow K_{0}^{\prime}[1]$ is an exact triangle in $W^{-}\left(\mathscr{Q}_{0}, \overline{\mathbb{Q}}_{\ell}\right)$, and two of the three complexes $K_{0}^{\prime}, K_{0}^{\prime \prime}$ and $K_{0}$ are convergent complexes, then so is the third, and $c_{v}\left(K_{0}\right)=c_{v}\left(K_{0}^{\prime}\right)+c_{v}\left(K_{0}^{\prime \prime}\right)$.

Proof. For every $x \in \mathscr{L}_{0}\left(\mathbb{F}_{q^{v}}\right)$, we have an exact triangle

$$
K_{\bar{x}}^{\prime} \longrightarrow K_{\bar{x}} \longrightarrow K_{\bar{x}}^{\prime \prime} \longrightarrow
$$

in $D_{c}^{-}\left(\overline{\mathbb{Q}}_{\ell}\right)$, equivariant under the action of $F_{x}$. Then apply Lemma 4.3.

Lemma 4.5. Theorem 4.2 holds for $f:$ Spec $\mathbb{F}_{q^{d}} \rightarrow \operatorname{Spec} \mathbb{F}_{q}$.

Proof. We have an equivalence of triangulated categories

$$
W^{-}\left(\operatorname{Spec} \mathbb{F}_{q}, \overline{\mathbb{Q}}_{\ell}\right) \stackrel{\sim}{\longrightarrow} D_{c}^{-}\left(\operatorname{Rep}_{\overline{\mathbb{Q}}_{\ell}} G\right),
$$

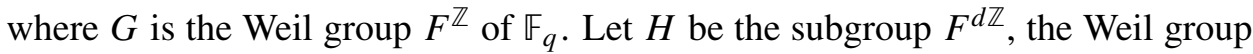
of $\mathbb{F}_{q^{d}}$. Since $f:$ Spec $\mathbb{F}_{q^{d}} \rightarrow \operatorname{Spec} \mathbb{F}_{q}$ is finite, we have $f_{!}=f_{*}$, and it is the induced-module functor

$$
\operatorname{Hom}_{\overline{\mathbb{Q}}_{\ell}[H]}\left(\overline{\mathbb{Q}}_{\ell}[G],-\right): D_{c}^{-}\left(\operatorname{Rep}_{\overline{\mathbb{Q}}_{\ell}} H\right) \longrightarrow D_{c}^{-}\left(\operatorname{Rep}_{\overline{\mathbb{Q}}_{\ell}} G\right),
$$

which is isomorphic to the coinduced-module functor $\overline{\mathbb{Q}}_{\ell}[G] \otimes_{\overline{\mathbb{Q}}_{\ell}[H]}-$. In particular, $f$ is exact on the level of sheaves.

Let $A$ be a $\overline{\mathbb{Q}}_{\ell}$-representation of $H$, and $B=\overline{\mathbb{Q}}_{\ell}[G] \otimes_{\overline{\mathbb{Q}}_{\ell}[H]} A$. Let $x_{1}, \ldots, x_{m}$ be an ordered basis for $A$ with respect to which $F^{d}$ is an upper triangular matrix

$$
\left[\begin{array}{ccc}
\alpha_{1} & * & * \\
& \ddots & * \\
& & \alpha_{m}
\end{array}\right]
$$


with eigenvalues $\alpha_{1}, \ldots, \alpha_{m}$. Then $B$ has an ordered basis

$$
\begin{array}{cccc}
1 \otimes x_{1}, & F \otimes x_{1}, & \cdots, & F^{d-1} \otimes x_{1}, \\
1 \otimes x_{2}, & F \otimes x_{2}, & \cdots, & F^{d-1} \otimes x_{2}, \\
\vdots & \vdots & \ddots & \vdots \\
1 \otimes x_{m}, & F \otimes x_{m}, & \cdots, & F^{d-1} \otimes x_{m},
\end{array}
$$

with respect to which $F$ is the matrix

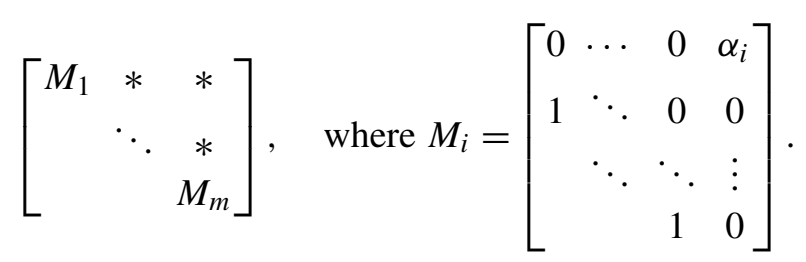

The characteristic polynomial of $F$ on $B$ is $\prod_{i=1}^{m}\left(t^{d}-\alpha_{i}\right)$.

Let $K_{0}$ be a complex of sheaves on Spec $\mathbb{F}_{q^{d}}$. The eigenvalues of the Frobenius $F$ on $\mathscr{H}^{n}\left(f_{!} K\right)=f_{!} \mathscr{H}^{n}(K)$ are all the $d$-th roots of the eigenvalues of $F^{d}$ on $\mathscr{H}^{n}(K)$, so for every $s>0$ we have

$$
\sum_{n} \sum_{\mathscr{H}^{n}\left(f_{!} K\right), F}|\alpha|^{s}=d \sum_{n} \sum_{\mathscr{H}^{n}(K), F^{d}}|\alpha|^{s / d} .
$$

This shows that $f_{!} K_{0}$ is a convergent complex on Spec $\mathbb{F}_{q}$ if and only if $K_{0}$ is a convergent complex on $\operatorname{Spec} \mathbb{F}_{q^{d}}$.

Next we prove

$$
c_{v}\left(\operatorname{Spec} \mathbb{F}_{q^{d}}, K_{0}\right)=c_{v}\left(\operatorname{Spec} \mathbb{F}_{q}, f_{!} K_{0}\right)
$$

for every $v \geq 1$. Since $H^{n}\left(f_{!} K\right)=f_{!} H^{n}(K)$, and both sides are absolutely convergent series so that one can change the order of summation without changing the limit, it suffices to prove it when $K=A$ is a single representation concentrated in degree 0 . Let us review this classical calculation. Use the notation above. For each $i$, fix a $d$-th root $\alpha_{i}^{1 / d}$ of $\alpha_{i}$, and let $\zeta_{d}$ be a primitive $d$-th root of unity. Then the eigenvalues of $F$ on $B$ are $\zeta_{d}^{k} \alpha_{i}^{1 / d}$, for $i=1, \ldots, m$ and $k=0, \ldots, d-1$.

If $d \nmid v$, then $\operatorname{Hom}_{\mathbb{F}_{q}}\left(\mathbb{F}_{q^{d}}, \mathbb{F}_{q^{v}}\right)=\varnothing$, so $c_{v}\left(\operatorname{Spec} \mathbb{F}_{q^{d}}, A\right)=0$. On the other hand,

$$
c_{v}\left(\operatorname{Spec} \mathbb{F}_{q}, f_{!} A\right)=\operatorname{Tr}\left(F^{v}, B\right)=\sum_{i, k} \zeta_{d}^{v k} \alpha_{i}^{v / d}=\sum_{i} \alpha_{i}^{v / d} \sum_{k=0}^{d-1} \zeta_{d}^{v k}=0 .
$$

If $d \mid v$, then $\operatorname{Hom}_{\mathbb{F}_{q}}\left(\mathbb{F}_{q^{d}}, \mathbb{F}_{q^{v}}\right)=\operatorname{Hom}_{\mathbb{F}_{q}}\left(\mathbb{F}_{q^{d}}, \mathbb{F}_{q^{d}}\right)=\mathbb{Z} / d \mathbb{Z}$. So

$$
c_{v}\left(\mathbb{F}_{q^{d}}, A\right)=d \operatorname{Tr}\left(F^{v}, A\right)=d \sum_{i} \alpha_{i}^{v / d}
$$


On the other hand,

$$
c_{v}\left(\mathbb{F}_{q}, B\right)=\operatorname{Tr}\left(F^{v}, B\right)=\sum_{i, k} \zeta_{d}^{v k} \alpha_{i}^{v / d}=\sum_{i, k} \alpha_{i}^{v / d}=d \sum_{i} \alpha_{i}^{v / d} .
$$

Next, we consider $B G_{0}$, for a finite group scheme $G_{0}$ over $\mathbb{F}_{q}$.

Lemma 4.6. Let $G_{0}$ be a finite $\mathbb{F}_{q}$-group scheme, and let $\mathscr{F}_{0}$ be a sheaf on $B G_{0}$. Then $H_{c}^{r}(B G, \mathscr{F})=0$ for all $r \neq 0$, and $H_{c}^{0}(B G, \mathscr{F}) \simeq H^{0}(B G, \mathscr{F})$ has dimension at most $\operatorname{rank}(\mathscr{F})$. Moreover, the set of $\iota$-weights of $H_{c}^{0}(B G, \mathscr{F})$ is a subset of the ı-weights of $\mathscr{F}_{0}$.

Proof. By [Olsson 2008a, 7.12-7.14] we can replace $G_{0}$ by its maximal reduced closed subscheme, and assume $G_{0}$ is reduced, hence étale. Then $G_{0}$ is the same as a finite group $G(\mathbb{F})$ with a continuous action of $\mathrm{Gal}\left(\mathbb{F}_{q}\right)$ [Milne 2012, XII, 2.11]. We will also write $G$ for the group $G(\mathbb{F})$, if there is no confusion. Since Spec $\mathbb{F} \rightarrow B G$ is surjective, we see that there is no nontrivial stratification on $B G$. In particular, all sheaves on $B G$ are lisse, and they are just $\overline{\mathbb{Q}}_{\ell}$-representations of $G$.

$B G$ is quasi-finite and proper over $\mathbb{F}$, with finite diagonal, so by [Olsson 2008a, 5.8], $H_{c}^{r}(B G, \mathscr{F})=0$ for all $r \neq 0$. From [ibid., 5.1], we see that if $\mathscr{F}$ is a sheaf on $B G$ corresponding to the representation $V$ of $G$, then $H_{c}^{0}(B G, \mathscr{F})=V_{G}$ and $H^{0}(B G, \mathscr{F})=V^{G}$, and there is a natural isomorphism

$$
v \mapsto \sum_{g \in G} g v: V_{G} \longrightarrow V^{G} .
$$

Therefore

$$
h_{c}^{0}(B G, \mathscr{F})=\operatorname{dim} V_{G} \leq \operatorname{dim} V=\operatorname{rank}(\mathscr{F}),
$$

and the weights of $V_{G}$ form a subset of the weights of $V$ (counted with multiplicities).

4.7. (i) If $k$ is a field, by a $k$-algebraic group $G$ we mean a smooth $k$-group scheme of finite type. If $G$ is connected, then it is geometrically connected [Grothendieck and Demazure 1970, Exposé $\left.\mathrm{VI}_{\mathrm{A}}, 2.1 .1\right]$.

(ii) For a connected $k$-algebraic group $G$, let $a: B G \rightarrow \operatorname{Spec} k$ be the structural map. Then

$$
a^{*}: \Lambda-\operatorname{Sh}(\operatorname{Spec} k) \longrightarrow \Lambda-\operatorname{Sh}(B G)
$$

is an equivalence of categories. This is because

- $B G$ has no nontrivial stratifications (it is covered by $\operatorname{Spec} k$ which has no nontrivial stratifications), and therefore

- any constructible $\Lambda$-adic sheaf on $B G$ is lisse, given by an adic system $\left(M_{n}\right)_{n}$ of sheaves on Spec $k$ with $G$-actions, and these actions are trivial since $G$ is connected, see [Behrend 2003, 5.2.9]. 
(iii) Let $G_{0}$ be a connected $\mathbb{F}_{q}$-algebraic group. By a theorem of Lang [1956, Theorem 2], every $G_{0}$-torsor over $\operatorname{Spec} \mathbb{F}_{q}$ is trivial, with automorphism group $G_{0}$, therefore

$$
c_{v}\left(B G_{0}\right)=\frac{1}{c_{v}\left(G_{0}\right)}=\frac{1}{\# G_{0}\left(\mathbb{F}_{q^{v}}\right)} .
$$

Recall the following theorem of Borel as in [Behrend 2003, 6.1.6].

Theorem 4.8. Let $k$ be a field and $G$ a connected $k$-algebraic group. Consider the Leray spectral sequence given by the projection $f: \operatorname{Spec} k \rightarrow B G$,

$$
E_{2}^{r s}=H^{r}\left(B G_{\bar{k}}\right) \otimes H^{s}\left(G_{\bar{k}}\right) \Longrightarrow \overline{\mathbb{Q}}_{\ell} .
$$

Let $N^{s}=E_{s+1}^{0, s} \subset H^{s}\left(G_{\bar{k}}\right)$ be the transgressive subspaces, for $s \geq 1$, and let $N$ be the graded $\overrightarrow{\mathbb{Q}}_{\ell}$-vector space $\bigoplus_{s \geq 1} N^{s}$. We have:

(a) $N^{s}=0$ if $s$ is even.

(b) The canonical map $\bigwedge N \rightarrow H^{*}\left(G_{\bar{k}}\right)$ is an isomorphism of graded $\overline{\mathbb{Q}}_{\ell}$-algebras.

(c) The spectral sequence above induces an epimorphism of graded $\overline{\mathbb{Q}}_{\ell}$-vector spaces $H^{*}\left(B G_{\bar{k}}\right) \rightarrow N[-1]$. Any section induces an isomorphism

$$
\operatorname{Sym}^{*}(N[-1]) \stackrel{\sim}{\longrightarrow} H^{*}\left(B G_{\bar{k}}\right) .
$$

Remark 4.8.1. (i) The $E_{2}^{r s}$-term in Theorem 4.8 should be $H^{r}\left(B G_{\bar{k}}, R^{s} f_{*} \overline{\mathbb{Q}}_{\ell}\right)$, and $R^{s} f_{*} \overline{\mathbb{Q}}_{\ell}$ is a constructible sheaf on $B G$. By 4.7 (ii), the sheaf $R^{s} f_{*} \overline{\mathbb{Q}}_{\ell}$ is isomorphic to $a^{*} f^{*} R^{s} f_{*} \overline{\mathbb{Q}}_{\ell}=a^{*} H^{s}\left(G_{\bar{k}}\right)$, where $a: B G \rightarrow$ Spec $k$ is the structural map and $H^{s}\left(G_{\bar{k}}\right)$ is the $\mathrm{Gal}(k)$-module regarded as a sheaf on Spec $k$. Therefore by projection formula, $E_{2}^{r s}=H^{r}\left(B G_{\bar{k}}\right) \otimes H^{s}\left(G_{\bar{k}}\right)$.

(ii) Since the spectral sequence converges to $\overline{\mathbb{Q}}_{\ell}$ sitting in degree 0 , all $E_{\infty}^{r s}$ are zero, except $E_{\infty}^{00}$. For each $s \geq 1$, consider the differential map

$$
d_{s+1}^{0, s}: E_{s+1}^{0, s} \longrightarrow E_{s+1}^{s+1,0}
$$

on the $(s+1)$ st page. This map must be injective (respectively surjective) because it is the last possibly nonzero map from $E_{*}^{0, s}$ (respectively into $E_{*}^{s+1,0}$ ). Therefore, it is an isomorphism. Here $N^{s}=E_{s+1}^{0, s}$ is a subspace of $E_{2}^{0, s}=H^{s}\left(G_{\bar{k}}\right)$, and $E_{s+1}^{s+1,0}$ is a quotient of $E_{2}^{s+1,0}=H^{s+1}\left(B G_{\bar{k}}\right)$. We get the surjection $H^{s+1}\left(B G_{\bar{k}}\right) \rightarrow N^{s}$ by using the isomorphism $d_{s+1}^{0, s}$.

4.8.2. Let $G_{0}$ be a connected $\mathbb{F}_{q}$-algebraic group of dimension $d$. We intend to apply Theorem 4.8 to investigate the compact support cohomology groups $H_{c}^{*}(B G)$.

We have graded Galois-invariant subspaces $N=\bigoplus_{r \geq 1} N^{r} \subset \bigoplus_{r \geq 0} H^{r}(G)$ concentrated in odd degrees, such that the induced map

$$
\bigwedge N \longrightarrow H^{*}(G)
$$


is an isomorphism, and such that $H^{*}(B G) \cong \operatorname{Sym}^{*} N[-1]$. Let $n_{r}=\operatorname{dim} N^{r}$, and let $v_{r 1}, \ldots, v_{r n_{r}}$ be a basis for $N^{r}$ with respect to which the Frobenius acting on $N^{r}$ is upper triangular

$$
\left[\begin{array}{ccc}
\alpha_{r 1} & * & * \\
& \ddots & * \\
& & \alpha_{r n_{r}}
\end{array}\right]
$$

with eigenvalues $\alpha_{r 1}, \ldots, \alpha_{r n_{r}}$. By Deligne [1980, 3.3.5], the weights of $H^{r}(G)$ are $\geq r$, so $\left|\alpha_{r i}\right| \geq q^{r / 2}>1$. We have

$$
\left.H^{*}(B G)=\operatorname{Sym}^{*} \overline{\mathbb{Q}}_{\ell}\left\langle v_{i j}\right| \text { for all } i, j\right\rangle=\overline{\mathbb{Q}}_{\ell}\left[v_{i j}\right],
$$

with $\operatorname{deg}\left(v_{i j}\right)=i+1$. Note that all $i+1$ are even. In particular, $H^{2 r-1}(B G)=0$ and

$$
\begin{aligned}
H^{2 r}(B G) & =\left\{\text { homogeneous polynomials of degree } 2 r \text { in } v_{i j}\right\} \\
& =\overline{\mathbb{Q}}_{\ell}\left\langle\prod_{i, j} v_{i j}^{m_{i j}} ; \sum_{i, j} m_{i j}(i+1)=2 r\right\rangle .
\end{aligned}
$$

With respect to an appropriate order of the basis, the matrix representing $F$ acting on $H^{2 r}(B G)$ is upper triangular, with eigenvalues

$$
\prod_{i, j} \alpha_{i j}^{m_{i j}}, \quad \text { for } \sum_{i, j} m_{i j}(i+1)=2 r .
$$

By Poincaré duality, the eigenvalues of $F$ acting on $H_{c}^{-2 r-2 d}(B G)$ are

$$
q^{-d} \prod_{i, j} \alpha_{i j}^{-m_{i j}}, \quad \text { where } \sum_{i, j} m_{i j}(i+1)=2 r .
$$

Here $\left(m_{i j}\right)_{i, j}$ are tuples of nonnegative integers. Therefore the reciprocal characteristic polynomial of $F$ on $H_{c}^{-2 r-2 d}(B G)$ is

$$
P_{-2 r-2 d}\left(B G_{0}, t\right)=\prod_{\substack{m_{i j} \geq 0 \\ \sum_{i, j} m_{i j}(i+1)=2 r}}\left(1-q^{-d} \prod_{i, j} \alpha_{i j}^{-m_{i j}} \cdot t\right) .
$$

In the following two lemmas we prove two key cases of Theorem 4.2(i).

Lemma 4.9. Let $G_{0}$ be an $\mathbb{F}_{q}$-group scheme of finite type. Then Theorem 4.2 (i) holds for the structural map $f: B G_{0} \rightarrow$ Spec $\mathbb{F}_{q}$ and any convergent complex $K_{0} \in W^{-}\left(B G_{0}, \overline{\mathbb{Q}}_{\ell}\right)$.

Proof. By Olsson [2008a, 7.12-7.14] we may assume that $G_{0}$ is reduced (hence smooth), so that the natural projection Spec $\mathbb{F}_{q} \rightarrow B G_{0}$ is a presentation. Note that 
then the assumptions of $\iota$-mixedness and stratifiability on $K_{0}$ are verified automatically, by Proposition 2.8 and Corollary 3.6 (iii), even though we will not use them explicitly in the proof.

Let $G_{0}^{0}$ be the identity component of $G_{0}$ and consider the exact sequence of algebraic groups

$$
1 \longrightarrow G_{0}^{0} \longrightarrow G_{0} \longrightarrow \pi_{0}\left(G_{0}\right) \longrightarrow 1
$$

The fibers of the induced map $B G_{0} \rightarrow B \pi_{0}\left(G_{0}\right)$ are isomorphic to $B G_{0}^{0}$, so we reduce to prove two cases: $G_{0}$ is finite étale (or even a finite constant group scheme, by Remark 4.1.2 (iii)), or $G_{0}$ is connected and smooth.

Case of $G_{0}$ finite constant. Let $G_{0} / \mathbb{F}_{q}$ be the finite constant group scheme associated with a finite group $G$, and let $K_{0} \in W^{-}\left(B G_{0}, \overline{\mathbb{Q}}_{\ell}\right)$. Again we denote by $G$ both the group scheme $G_{0} \otimes \mathbb{F}$ and the finite group $G_{0}(\mathbb{F})$, if no confusion arises. Let $y$ be the unique point in $\operatorname{Spec} \mathbb{F}_{q}$,

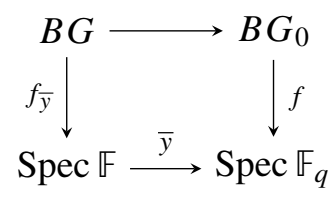

is identified with the coinvariance functor

$$
()_{G}: D_{c}^{-}\left(\operatorname{Rep}_{\overline{\mathbb{Q}}_{\ell}} G\right) \longrightarrow D_{c}^{-}\left(\overline{\mathbb{Q}}_{\ell}\right),
$$

which is exact on the level of modules, since the category $\operatorname{Rep}_{\bar{Q}_{\ell}} G$ is semisimple. So $\left(f_{!} K_{0}\right)_{\bar{y}}=\left(f_{\bar{y}}\right)_{!} K=K_{G}$ and $\mathscr{H}^{n}\left(K_{G}\right)=\mathscr{H}^{n}(K)_{G}$. Therefore

$$
\sum_{\mathscr{H e}^{n}\left(\left(f_{\bar{y}) !}\right), F\right.}|\alpha|^{s} \leq \sum_{\mathscr{H e}^{n}(K), F}|\alpha|^{s}
$$

for every $n \in \mathbb{Z}$ and $s>0$. Therefore, if $K_{0}$ is a convergent complex, so is $f_{!} K_{0}$.

Case of $G_{0}$ smooth and connected. In this case

$$
f^{*}: \overline{\mathbb{Q}}_{\ell}-\operatorname{Sh}\left(\operatorname{Spec} \mathbb{F}_{q}\right) \longrightarrow \overline{\mathbb{Q}}_{\ell}-\operatorname{Sh}\left(B G_{0}\right)
$$

is an equivalence of categories by 4.7 (ii). Let $d=\operatorname{dim} G_{0}$, and let $\mathscr{F}_{0}$ be a sheaf on $B G_{0}$, corresponding to a representation $V$ of the Weil group $W\left(\mathbb{F}_{q}\right)$, with $\beta_{1}, \ldots, \beta_{m}$ as eigenvalues of $F$. By the projection formula [Laszlo and Olsson 2008b, 9.1.i] we have $H_{c}^{n}(B G, \mathscr{F}) \simeq H_{c}^{n}(B G) \otimes V$, and by 4.8.2 the eigenvalues of $F$ on $H_{c}^{-2 r-2 d}(B G) \otimes V$ are (using the notation in 4.8.2)

$$
q^{-d} \beta_{k} \prod_{i, j} \alpha_{i j}^{-m_{i j}}
$$


for $k=1, \ldots, m$ and tuples $\left(m_{i j}\right)$ such that $\sum_{i, j} m_{i j}(i+1)=2 r$. For every $s>0$,

$$
\begin{aligned}
\sum_{n \in \mathbb{Z}} \sum_{H_{c}^{n}(B G) \otimes V, F}|\alpha|^{s} & =\sum_{m_{i j}, k} q^{-d s}\left|\beta_{k}\right|^{s} \prod_{i, j}\left|\alpha_{i j}^{-m_{i j}}\right|^{s} \\
& =\left(\sum_{k=1}^{m}\left|\beta_{k}\right|^{s}\right)\left(\sum_{m_{i j}} q^{-d s} \prod_{i, j}\left|\alpha_{i j}\right|^{-m_{i j} s}\right),
\end{aligned}
$$

which converges to

$$
q^{-d s}\left(\sum_{k=1}^{m}\left|\beta_{k}\right|^{s}\right) \prod_{i, j} \frac{1}{1-\left|\alpha_{i j}\right|^{-s}},
$$

since $\left|\alpha_{i j}\right|^{-s}<1$ and the product above is taken over finitely many indices.

Let $K_{0}$ be a convergent complex on $B G_{0}$, and for each $k \in \mathbb{Z}$, let $V_{k}$ be a $W\left(\mathbb{F}_{q}\right)$ module corresponding to $\mathscr{H}^{k} K_{0}$. For every $x \in B G_{0}\left(\mathbb{F}_{q}\right)$ (for instance the trivial $G_{0}$-torsor), the pair $\left(\mathscr{H}^{k}(K)_{\bar{x}}, F_{x}\right)$ is isomorphic to $\left(V_{k}, F\right)$. Consider the $W\left(\mathbb{F}_{q}\right)$ equivariant spectral sequence

$$
H_{c}^{r}\left(B G, \mathscr{H}^{k}(K)\right) \Longrightarrow H_{c}^{r+k}(B G, K) .
$$

We have

$$
\begin{aligned}
\sum_{n \in \mathbb{Z}} \sum_{H_{c}^{n}(B G, K), F}|\alpha|^{s} & \leq \sum_{n \in \mathbb{Z}} \sum_{r+k=n} \sum_{H_{c}^{r}\left(B G, \mathscr{F}^{k} K\right), F}|\alpha|^{s} \\
& =\sum_{r, k \in \mathbb{Z}} \sum_{H_{c}^{r}(B G) \otimes V_{k}, F}|\alpha|^{s} \\
& =\sum_{k \in \mathbb{Z}} \sum_{r \in \mathbb{Z}} \sum_{H_{c}^{r}(B G) \otimes V_{k}, F}|\alpha|^{s} \\
& =\sum_{k \in \mathbb{Z}} q^{-d s}\left(\sum_{V_{k}, F}|\alpha|^{s}\right) \prod_{i, j} \frac{1}{1-\left|\alpha_{i j}\right|^{-s}} \\
& =\left(\sum_{k \in \mathbb{Z}} \sum_{V_{k}, F}|\alpha|^{s}\right)\left(q^{-d s} \prod_{i, j} \frac{1}{1-\left|\alpha_{i j}\right|^{-s}}\right),
\end{aligned}
$$

where the first factor is convergent by assumption, and the product in the second factor is taken over finitely many indices. This shows that $f_{!} K_{0}$ is a convergent complex.

Let $E_{\lambda}$ be a finite subextension of $\overline{\mathbb{Q}}_{\ell} / \mathbb{Q}_{\ell}$ with ring of integers $\Lambda$ and residue field $\Lambda_{0}$, and let $(\mathscr{S}, \mathscr{L})$ be a pair on $\mathscr{L}$ defined by simple lcc $\Lambda_{0}$-sheaves on strata. A complex $K_{0} \in W\left(\mathscr{X}_{0}, \overline{\mathbb{Q}}_{\ell}\right)$ is said to be $(\mathscr{Y}, \mathscr{L})$-stratifiable (or trivialized by $(\mathscr{Y}, \mathscr{L})$ ), if $K$ is defined over $E_{\lambda}$, with an integral model over $\Lambda$ trivialized by $(\mathscr{Y}, \mathscr{L})$. 
Lemma 4.10. Let $X_{0} / \mathbb{F}_{q}$ be a geometrically connected variety, $E_{\lambda}$ a finite subextension of $\overline{\mathbb{Q}}_{\ell} / \mathbb{Q}_{\ell}$ with ring of integers $\Lambda$, and let $\mathscr{L}$ be a finite set of simple lcc $\Lambda_{0}$-sheaves on $X$. Then Theorem 4.2 (i) holds for the structural morphism $f: X_{0} \rightarrow$ Spec $\mathbb{F}_{q}$ and all lisse -mixed convergent complexes $K_{0}$ on $X_{0}$ that are trivialized by $(\{X\}, \mathscr{L})$.

Proof. Let $N=\operatorname{dim} X_{0}$. From the spectral sequence

$$
E_{2}^{r k}=H_{c}^{r}\left(X, \mathscr{H}^{k} K\right) \Longrightarrow H_{c}^{r+k}(X, K)
$$

we see that

$$
\sum_{n \in \mathbb{Z}} \sum_{H_{c}^{n}(X, K), F}|\alpha|^{s} \leq \sum_{n \in \mathbb{Z}} \sum_{r+k=n} \sum_{H_{c}^{r}\left(X, \mathscr{H}^{k} K\right), F}|\alpha|^{s}=\sum_{\substack{0 \leq r \leq 2 N \\ k \in \mathbb{Z}}} \sum_{H_{c}^{r}\left(X, \mathscr{K ^ { k }} K\right), F}|\alpha|^{s},
$$

therefore it suffices to show that the series $\sum_{k \in \mathbb{Z}} \sum_{H_{c}^{r}\left(X, \mathscr{H}^{k} K\right), F}|\alpha|^{s}$ converges for each $0 \leq r \leq 2 N$.

Let $d$ be the number in Lemma 3.10 for $\mathscr{L}$. Each cohomology sheaf $\mathscr{H}^{n} K_{0}$ is $\iota$-mixed and lisse on $X_{0}$, so by Theorem 2.6 (i) we have the decomposition

$$
\mathscr{H}^{n} K_{0}=\bigoplus_{b \in \mathbb{R} / \mathbb{Z}}\left(\mathscr{H}^{n} K_{0}\right)(b)
$$

according to the weights $\bmod \mathbb{Z}$, defined over $E_{\lambda}$, see Remark 2.6.1 (ii). For each coset $b$, we choose a representative $b_{0} \in b$, and take a $b_{1} \in \overline{\mathbb{Q}}_{\ell}^{*}$ such that $w_{q}\left(b_{1}\right)=-b_{0}$. Then the sheaf $\left(\mathscr{H}^{n} K_{0}\right)(b)^{\left(b_{1}\right)}$ deduced by twist is lisse with integer punctual weights. Let $W$ be the filtration by punctual weights Theorem 2.6 (ii) of $\left(\mathscr{H}^{n} K_{0}\right)(b)^{\left(b_{1}\right)}$. For every $v \geq 1$ and $x \in X_{0}\left(\mathbb{F}_{q^{v}}\right)$, and every real $s>0$, we have

$$
\begin{aligned}
\sum_{n \in \mathbb{Z}} \sum_{\left(\mathscr{H}^{n} K_{0}\right)_{\bar{x}}, F_{x}}|\alpha|^{s / v} & =\sum_{\substack{n \in \mathbb{Z} \\
b \in \mathbb{R} / \mathbb{Z}}} \sum_{\left(\mathscr{H}^{n} K_{0}\right)(b)_{\bar{x}}, F_{x}}|\alpha|^{s / v} \\
& =\sum_{\substack{n \in \mathbb{Z} \\
b \in \mathbb{R} / \mathbb{Z}}} \sum_{\left(\mathscr{H}^{n} K_{0}\right)(b)_{\bar{x}}^{\left(b_{1}\right)}, F_{x}}\left|\alpha / b_{1}^{v}\right|^{s / v} \\
& =\sum_{\substack{n \in \mathbb{Z} \\
b \in \mathbb{R} / \mathbb{Z}}} q^{b_{0} s / 2} \sum_{\left(\mathscr{H}^{n} K_{0}\right)(b)_{\bar{x}}^{\left(b_{1}\right)}, F_{x}}|\alpha|^{s / v} \\
& =\sum_{\substack{n \in \mathbb{Z} \\
b \in \mathbb{R} / \mathbb{Z}}} q^{b_{0} s / 2} \sum_{i \in \mathbb{Z} \operatorname{Gr}_{i}^{W}\left(\left(\mathscr{C}^{n} K_{0}\right)(b)^{\left(b_{1}\right)}\right)_{\bar{x}}, F_{x}}|\alpha|^{s / v} \\
& =\sum_{\substack{n \in \mathbb{Z} \\
b \in \mathbb{R} / \mathbb{Z}}} q^{b_{0} s / 2} \sum_{i \in \mathbb{Z}} q^{i s / 2} \cdot \operatorname{rank} \operatorname{Gr}_{i}^{W}\left(\mathscr{H}^{n} K_{0}\right)(b)^{\left(b_{1}\right)} .
\end{aligned}
$$

Since $K_{0}$ is a convergent complex, this series is convergent. 
For each $n \in \mathbb{Z}$, every direct summand $\left(\mathscr{H}^{n} K_{0}\right)(b)$ of $\mathscr{H}^{n} K_{0}$ is trivialized by $(\{X\}, \mathscr{L})$. The filtration $W$ of each $\left(\mathscr{H}^{n} K_{0}\right)(b)^{\left(b_{1}\right)}$ gives a filtration of $\left(\mathscr{H}^{n} K_{0}\right)(b)$ (also denoted $W$ ) by twisting back, and it is clear that this latter filtration is defined over $E_{\lambda}$. We have $\operatorname{Gr}_{i}^{W}\left(\left(\mathscr{H}^{n} K_{0}\right)(b)^{\left(b_{1}\right)}\right)=\left(\mathrm{Gr}_{i}^{W}\left(\mathscr{H}^{n} K_{0}\right)(b)\right)^{\left(b_{1}\right)}$, and each $\mathrm{Gr}_{i}^{W}\left(\mathscr{H}^{n} K_{0}\right)(b)$ is trivialized by $(\{X\}, \mathscr{L})$. By Lemma 3.10,

$$
\begin{aligned}
h_{c}^{r}\left(X, \operatorname{Gr}_{i}^{W}\left(\left(\mathscr{H}^{n} K\right)(b)^{\left(b_{1}\right)}\right)\right) & =h_{c}^{r}\left(X, \operatorname{Gr}_{i}^{W}\left(\mathscr{H}^{n} K\right)(b)\right) \\
& \leq d \cdot \operatorname{rank} \operatorname{Gr}_{i}^{W}\left(\mathscr{H}^{n} K\right)(b) \\
& =d \cdot \operatorname{rank} \operatorname{Gr}_{i}^{W}\left(\left(\mathscr{H}^{n} K\right)(b)^{\left(b_{1}\right)}\right),
\end{aligned}
$$

where the first equality follows from [Laszlo and Olsson 2008b, 9.1.i]. Therefore

$$
\begin{aligned}
& \sum_{n \in \mathbb{Z}} \sum_{H_{c}^{r}\left(X, \mathscr{H}^{n} K\right), F}|\alpha|^{s}=\sum_{\substack{n \in \mathbb{Z} \\
b \in \mathbb{R} / \mathbb{Z}}} \sum_{H_{c}^{r}\left(X,\left(\mathscr{C}^{n} K\right)(b)\right), F}|\alpha|^{s} \\
& =\sum_{\substack{n \in \mathbb{Z} \\
b \in \mathbb{R} / \mathbb{Z}}} \sum_{H_{c}^{r}\left(X,\left(\mathscr{H}^{n} K\right)(b)^{\left(b_{1}\right)}\right), F}\left|b_{1}^{-1} \alpha\right|^{s} \\
& \leq \sum_{\substack{n \in \mathbb{Z} \\
b \in \mathbb{R} / \mathbb{Z}}} q^{b_{0} s / 2} \sum_{i \in \mathbb{Z}} \sum_{H_{c}^{r}\left(X, \mathrm{Gr}_{i}^{W}\left(\left(\mathscr{H}^{n} K\right)(b)^{\left(b_{1}\right)}\right)\right), F}|\alpha|^{s} \\
& \leq \sum_{\substack{n \in \mathbb{Z} \\
b \in \mathbb{R} / \mathbb{Z}}} q^{b_{0} s / 2} \sum_{i \in \mathbb{Z}} q^{(i+r) s / 2} \cdot h_{c}^{r}\left(X, \operatorname{Gr}_{i}^{W}\left(\left(\mathscr{H}^{n} K\right)(b)^{\left(b_{1}\right)}\right)\right) \\
& \leq q^{r s / 2} d \sum_{\substack{n \in \mathbb{Z} \\
b \in \mathbb{R} / \mathbb{Z}}} q^{b_{0} s / 2} \sum_{i \in \mathbb{Z}} q^{i s / 2} \cdot \operatorname{rank}_{i} \operatorname{Gr}_{i}^{W}\left(\left(\mathcal{H}^{n} K\right)(b)^{\left(b_{1}\right)}\right),
\end{aligned}
$$

and it converges.

Now we prove Theorem 4.2 (i) in general.

Proof. We may assume all stacks involved are reduced. From Theorem 2.11 and Theorem 3.9 we know that $f_{!} K_{0} \in W_{m}^{- \text {,stra }}\left(\mathscr{Y}_{0}, \overline{\mathbb{Q}}_{\ell}\right)$.

Let $y \in \mathscr{Y}_{0}\left(\mathbb{F}_{q^{v}}\right)$, we want to show that $\left(\left(f_{!} K_{0}\right)_{\bar{y}}, F_{y}\right)$ is a convergent complex. Since the property of being convergent depends only on the cohomology sheaves, by base change [Laszlo and Olsson 2008b, 12.5.3] we reduce to the case when $\mathscr{Y}_{0}=\operatorname{Spec} \mathbb{F}_{q^{v}}$. Replacing $q$ by $q^{v}$, we may assume $v=1$. By Remark 4.1.2 (iii) we only need to show that $\left(R \Gamma_{c}(\mathscr{X}, K), F\right)$ is convergent.

If $j: \mathscr{U}_{0} \hookrightarrow \mathscr{L}_{0}$ is an open substack with complement $i: \mathscr{L}_{0} \hookrightarrow \mathscr{X}_{0}$, then we have an exact triangle

$$
j ! j^{*} K_{0} \longrightarrow K_{0} \longrightarrow i_{*} i^{*} K_{0} \longrightarrow
$$


in $W^{-}\left(\mathscr{Q}_{0}, \overline{\mathbb{Q}}_{\ell}\right)$, which induces an exact triangle

$$
R \Gamma_{c}\left(\mathscr{U}_{0}, j^{*} K_{0}\right) \longrightarrow R \Gamma_{c}\left(\mathscr{C}_{0}, K_{0}\right) \longrightarrow R \Gamma_{c}\left(\mathscr{E}_{0}, i^{*} K_{0}\right) \longrightarrow
$$

in $W^{-}\left(\operatorname{Spec} \mathbb{F}_{q}, \overline{\mathbb{Q}}_{\ell}\right)$. So by Corollary 4.4 and noetherian induction, it suffices to prove Theorem 4.2 (i) for a nonempty open substack. By [Behrend 2003, 5.1.14] we may assume that the inertia stack $\mathscr{I}_{0}$ is flat over $\mathscr{L}_{0}$. Then we can form the rigidification $\pi: \mathscr{X}_{0} \rightarrow X_{0}$ with respect to $\mathscr{I}_{0}$ [Olsson 2008b, §1.5], where $X_{0}$ is an $\mathbb{F}_{q}$-algebraic space of quasi-compact diagonal. $X_{0}$ contains an open dense subscheme [Knutson 1971, II, 6.7]. Replacing $\mathscr{X}_{0}$ by the inverse image of this scheme, we can assume $X_{0}$ is a scheme.

If Theorem 4.2 (i) holds for two composable morphisms $f$ and $g$, then it holds for their composition $g \circ f$. Since $R \Gamma_{c}\left(\mathscr{C}_{0},-\right)=R \Gamma_{c}\left(X_{0},-\right) \circ \pi_{!}$, we reduce to proving Theorem 4.2 (i) for these two morphisms. For every $x \in X_{0}\left(\mathbb{F}_{q^{v}}\right)$, the fiber of $\pi$ over $x$ is a gerbe over $\operatorname{Spec} k(x)$. Extending the base $k(x)$ (see Remark 4.1.2(iii)) one can assume it is a neutral gerbe (in fact all gerbes over a finite field are neutral; see [Behrend 2003, 6.4.2]). This means the following diagram is 2-Cartesian:

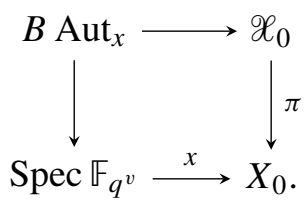

So we reduce to two cases: $\mathscr{L}_{0}=B G_{0}$ for an $\mathbb{F}_{q}$-algebraic group $G_{0}$, or $\mathscr{L}_{0}=X_{0}$ is an $\mathbb{F}_{q}$-scheme. The first case is proved in Lemma 4.9.

For the second case, given a convergent complex $K_{0} \in W_{m}^{- \text {,stra }}\left(X_{0}, \overline{\mathbb{Q}}_{\ell}\right)$, defined over some $E_{\lambda}$ with ring of integers $\Lambda$, and trivialized by a pair $(\mathscr{Y}, \mathscr{L})(\mathscr{L}$ being defined over $\Lambda_{0}$ ) on $X$, we can refine this pair so that every stratum is connected, and replace $X_{0}$ by models of the strata defined over some finite extension of $\mathbb{F}_{q}$ Remark 4.1.2 (iii). This case is proved in Lemma 4.10.

\section{Trace formula for stacks}

We prove two special cases of Theorem 4.2 (ii) in the following two propositions.

Proposition 5.1. Let $G_{0}$ be a finite étale group scheme over $\mathbb{F}_{q}$, and $\mathscr{F}_{0}$ a sheaf on $B G_{0}$. Then

$$
c_{1}\left(B G_{0}, \mathscr{F}_{0}\right)=c_{1}\left(\operatorname{Spec} \mathbb{F}_{q}, R \Gamma_{c}\left(B G_{0}, \mathscr{F}_{0}\right)\right) .
$$

Proof. This is a special case of [Olsson 2008a, 8.6] on correspondences given by group homomorphisms. 
Proposition 5.2. Let $G_{0}$ be a connected $\mathbb{F}_{q}$-algebraic group, and let $\mathscr{F}_{0}$ be a sheaf on $B G_{0}$. Then

$$
c_{1}\left(B G_{0}, \mathscr{F}_{0}\right)=c_{1}\left(\operatorname{Spec} \mathbb{F}_{q}, R \Gamma_{c}\left(B G_{0}, \mathscr{F}_{0}\right)\right) .
$$

Proof. Let $f: B G_{0} \rightarrow \operatorname{Spec} \mathbb{F}_{q}$ be the structural map and $d=\operatorname{dim} G_{0}$. By 4.7 (ii), the sheaf $\mathscr{F}_{0}$ on $B G_{0}$ takes the form $f^{*} V$, for some sheaf $V$ on Spec $\mathbb{F}_{q}$. By 4.7 (iii), we have

$$
c_{1}\left(B G_{0}, \mathscr{F}_{0}\right)=\frac{1}{\# G_{0}\left(\mathbb{F}_{q}\right)} \operatorname{Tr}\left(F_{x}, \mathscr{F}_{\bar{x}}\right)=\frac{\operatorname{Tr}(F, V)}{\# G_{0}\left(\mathbb{F}_{q}\right)} .
$$

By the projection formula we have $H_{c}^{n}(B G, \mathscr{F}) \simeq H_{c}^{n}(B G) \otimes V$, so

$$
\operatorname{Tr}\left(F, H_{c}^{n}(B G, \mathscr{F})\right)=\operatorname{Tr}\left(F, H_{c}^{n}(B G)\right) \cdot \operatorname{Tr}(F, V) .
$$

Then

$$
\begin{aligned}
c_{1}\left(\operatorname{Spec} \mathbb{F}_{q}, R \Gamma_{c}\left(B G_{0}, \mathscr{F}_{0}\right)\right) & =\sum_{n}(-1)^{n} \operatorname{Tr}\left(F, H_{c}^{n}(B G, \mathscr{F})\right) \\
& =\operatorname{Tr}(F, V) \sum_{n}(-1)^{n} \operatorname{Tr}\left(F, H_{c}^{n}(B G)\right),
\end{aligned}
$$

so we can assume $\mathscr{F}_{0}=\overline{\mathbb{Q}}_{\ell}$. Using the notations in 4.8.2 we have

$$
\begin{gathered}
\sum_{n}(-1)^{n} \operatorname{Tr}\left(F, H_{c}^{n}(B G)\right)=\sum_{r \geq 0} \operatorname{Tr}\left(F, H_{c}^{-2 r-2 d}(B G)\right) \\
=\sum_{r \geq 0} \sum_{\begin{array}{c}
\sum_{i j}(i+1)=2 r \\
m_{i j} \geq 0
\end{array}} q^{-d} \prod_{i, j} \alpha_{i j}^{-m_{i j}}=q^{-d} \sum_{m_{i j} \geq 0} \prod_{i, j} \alpha_{i j}^{-m_{i j}} \\
=q^{-d} \prod_{i, j}\left(1+\alpha_{i j}^{-1}+\alpha_{i j}^{-2}+\cdots\right)=q^{-d} \prod_{i, j} \frac{1}{1-\alpha_{i j}^{-1}} .
\end{gathered}
$$

It remains to show that

$$
\# G_{0}\left(\mathbb{F}_{q}\right)=q^{d} \prod_{i, j}\left(1-\alpha_{i j}^{-1}\right) .
$$

In 4.8.2, we saw that if each $N^{i}$ has an ordered basis $v_{i 1}, \ldots, v_{i n_{i}}$ with respect to which $F$ is upper triangular, then since $H^{*}(G)=\bigwedge N, H^{i}(G)$ has a basis

$$
v_{i_{1} j_{1}} \wedge v_{i_{2} j_{2}} \wedge \cdots \wedge v_{i_{m} j_{m}},
$$

such that $\sum_{r=1}^{m} i_{r}=i, i_{r} \leq i_{r+1}$, and if $i_{r}=i_{r+1}$, then $j_{r}<j_{r+1}$. The eigenvalues of $F$ on $H^{i}(G)$ are $\alpha_{i_{1} j_{1}} \cdots \alpha_{i_{m} j_{m}}$ for such indices. By Poincaré duality, the eigenvalues of $F$ on $H_{c}^{2 d-i}(G)$ are $q^{d}\left(\alpha_{i_{1} j_{1}} \cdots \alpha_{i_{m} j_{m}}\right)^{-1}$. Note that all the $i_{r}$ are odd, so

$$
2 d-i \equiv i=\sum_{r=1}^{m} i_{r} \equiv m \quad \bmod 2 .
$$


Applying the classical trace formula to $G_{0}$, we have

$$
\# G_{0}\left(\mathbb{F}_{q}\right)=\sum(-1)^{m} q^{d} \alpha_{i_{1} j_{1}}^{-1} \cdots \alpha_{i_{m} j_{m}}^{-1}=q^{d} \prod_{i, j}\left(1-\alpha_{i j}^{-1}\right) .
$$

This finishes the proof.

5.2.1. Note that in Propositions 5.1 and 5.2 we did not make explicit use of the fact that $\mathscr{F}_{0}$ is $\iota$-mixed.

Now we prove Theorem 4.2 (ii) in general.

Proof. Since $c_{v}\left(\mathscr{X}_{0}, K_{0}\right)=c_{1}\left(\mathscr{X}_{0} \otimes \mathbb{F}_{q^{v}}, K_{0} \otimes \mathbb{F}_{q^{v}}\right)$, we can assume $v=1$. We shall reduce to proving Theorem 4.2 (ii) for all fibers of $f$ over $\mathbb{F}_{q}$-points of $\mathscr{Y}_{0}$, following the approach of Behrend [2003, 6.4.9].

Let $y \in \mathscr{Y}_{0}\left(\mathbb{F}_{q}\right)$ and $\left(\mathscr{X}_{0}\right)_{y}$ be the fiber over $y$. Then $\left(\mathscr{L}_{0}\right)_{y}\left(\mathbb{F}_{q}\right)$ is the groupoid of pairs $(x, \alpha)$, where $x \in \mathscr{X}_{0}\left(\mathbb{F}_{q}\right)$ and $\alpha: f(x) \rightarrow y$ is an isomorphism in $\mathscr{Y}_{0}\left(\mathbb{F}_{q}\right)$. Suppose $\left(\mathscr{L}_{0}\right)_{y}\left(\mathbb{F}_{q}\right) \neq \varnothing$, and fix an $x \in\left(\mathscr{X}_{0}\right)_{y}\left(\mathbb{F}_{q}\right)$. Then $\operatorname{Isom}(f(x), y)\left(\mathbb{F}_{q}\right)$ is a trivial left $\operatorname{Aut}_{y}\left(\mathbb{F}_{q}\right)$-torsor. There is also a natural right action of $\operatorname{Aut}_{x}\left(\mathbb{F}_{q}\right)$ on Isom $(f(x), y)\left(\mathbb{F}_{q}\right)$, namely $\varphi \in \operatorname{Aut}_{x}\left(\mathbb{F}_{q}\right)$ takes $\alpha$ to $\alpha \circ f(\varphi)$. Under this action, for $\alpha$ and $\alpha^{\prime}$ to be in the same orbit, there should be a $\varphi \in \operatorname{Aut}_{x}\left(\mathbb{F}_{q}\right)$ such that the diagram

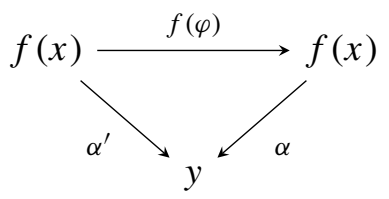

commutes; by definition this means $(x, \alpha)$ is isomorphic to $\left(x, \alpha^{\prime}\right)$ in $\left(\mathscr{C}_{0}\right)_{y}\left(\mathbb{F}_{q}\right)$. So the set of orbits $\operatorname{Isom}(f(x), y)\left(\mathbb{F}_{q}\right) / \operatorname{Aut}_{x}\left(\mathbb{F}_{q}\right)$ is identified with the inverse image of the class of $x$ along the map $\left[\left(\mathscr{C}_{0}\right)_{y}\left(\mathbb{F}_{q}\right)\right] \rightarrow\left[\mathscr{Q}_{0}\left(\mathbb{F}_{q}\right)\right]$. The stabilizer group of $\alpha \in \operatorname{Isom}(f(x), y)\left(\mathbb{F}_{q}\right)$ is $\operatorname{Aut}_{(x, \alpha)}\left(\mathbb{F}_{q}\right)$, the automorphism group of $(x, \alpha)$ in $\left(\mathscr{X}_{0}\right)_{y}\left(\mathbb{F}_{q}\right)$.

In general, if a finite group $G$ acts on a finite set $S$, then we have

$$
\sum_{[x] \in S / G} \frac{\# G}{\# \operatorname{Stab}_{G}(x)}=\sum_{[x] \in S / G} \# \operatorname{Orb}_{G}(x)=\# S .
$$

Now for $S=\operatorname{Isom}(f(x), y)\left(\mathbb{F}_{q}\right)$ and $G=\operatorname{Aut}_{x}\left(\mathbb{F}_{q}\right)$, we have

$$
\sum_{\substack{(x, \alpha) \in\left[\left(\mathscr{Q}_{0}\right)_{y}\left(\mathbb{F}_{q}\right)\right] \\(x, \alpha) \mapsto x}} \frac{\# \operatorname{Aut}_{x}\left(\mathbb{F}_{q}\right)}{\# \operatorname{Aut}_{(x, \alpha)}\left(\mathbb{F}_{q}\right)}=\# \operatorname{Isom}(f(x), y)\left(\mathbb{F}_{q}\right)=\# \operatorname{Aut}_{y}\left(\mathbb{F}_{q}\right) ;
$$

the last equality follows from the fact that $S$ is a trivial Aut $y\left(\mathbb{F}_{q}\right)$-torsor. 
If we assume Theorem 4.2 (ii) holds for the fibers $f_{y}:\left(\mathscr{C}_{0}\right)_{y} \rightarrow \operatorname{Spec} \mathbb{F}_{q}$ of $f$, for all $y \in \mathscr{Y}_{0}\left(\mathbb{F}_{q}\right)$, then

$$
\begin{aligned}
& c_{1}\left(\mathscr{Y}_{0}, f_{!} K_{0}\right)=\sum_{y \in\left[\mathscr{Y}_{0}\left(\mathbb{F}_{q}\right)\right]} \frac{\operatorname{Tr}\left(F_{y},\left(f_{!} K\right)_{\bar{y}}\right)}{\# \operatorname{Aut}_{y}\left(\mathbb{F}_{q}\right)} \\
& =\sum_{y \in\left[\mathscr{Y}_{0}\left(\mathbb{F}_{q}\right)\right]} \frac{\operatorname{Tr}\left(F_{y},\left(f_{y !} K\right)_{\bar{y}}\right)}{\# \operatorname{Aut}_{y}\left(\mathbb{F}_{q}\right)} \\
& =\sum_{y \in\left[\mathscr{Y}_{0}\left(\mathbb{F}_{q}\right)\right]} \frac{1}{\# \operatorname{Aut}_{y}\left(\mathbb{F}_{q}\right)} \sum_{(x, \alpha) \in\left[\left(\mathscr{Q}_{0}\right)_{y}\left(\mathbb{F}_{q}\right)\right]} \frac{\operatorname{Tr}\left(F_{x}, K_{\bar{x}}\right)}{\# \operatorname{Aut}_{(x, \alpha)}\left(\mathbb{F}_{q}\right)} \\
& =\sum_{y \in\left[\mathscr{Y}_{0}\left(\mathbb{F}_{q}\right)\right]} \frac{1}{\# \operatorname{Aut}_{y}\left(\mathbb{F}_{q}\right)} \sum_{\substack{x \in\left[\mathscr{Q}_{0}\left(\mathbb{F}_{q}\right)\right] \\
x \mapsto y}}\left(\sum_{\substack{(x, \alpha) \in\left[\left(\mathscr{Q}_{0}\right)_{y}\left(\mathbb{F}_{q}\right)\right] \\
(x, \alpha) \mapsto x}} \frac{\operatorname{Tr}\left(F_{x}, K_{\bar{x}}\right)}{\# \operatorname{Aut}_{(x, \alpha)}\left(\mathbb{F}_{q}\right)}\right) \\
& =\sum_{y \in\left[\mathscr{Y}_{0}\left(\mathbb{F}_{q}\right)\right]} \frac{1}{\# \operatorname{Aut}_{y}\left(\mathbb{F}_{q}\right)} \sum_{\substack{x \in\left[\mathscr{Q}_{0}\left(\mathbb{F}_{q}\right)\right] \\
x \mapsto y}} \frac{1}{\# \operatorname{Aut}_{x}\left(\mathbb{F}_{q}\right)} \\
& \left(\sum_{\substack{(x, \alpha) \in\left[\left(\mathscr{Q}_{0}\right)_{y}\left(\mathbb{F}_{q}\right)\right] \\
(x, \alpha) \mapsto x}} \frac{\# \operatorname{Aut}_{x}\left(\mathbb{F}_{q}\right)}{\# \operatorname{Aut}_{(x, \alpha)}\left(\mathbb{F}_{q}\right)}\right) \operatorname{Tr}\left(F_{x}, K_{\bar{x}}\right) \\
& =\sum_{y \in\left[\mathscr{Y}_{0}\left(\mathbb{F}_{q}\right)\right]} \frac{1}{\# \operatorname{Aut}_{y}\left(\mathbb{F}_{q}\right)} \sum_{\substack{x \in\left[\mathscr{L}_{0}\left(\mathbb{F}_{q}\right)\right] \\
x \mapsto y}} \frac{\operatorname{Tr}\left(F_{x}, K_{\bar{x}}\right)}{\# \operatorname{Aut}_{x}\left(\mathbb{F}_{q}\right)} \# \operatorname{Aut}_{y}\left(\mathbb{F}_{q}\right) \\
& =\sum_{x \in\left[\mathscr{L}_{0}\left(\mathbb{F}_{q}\right)\right]} \frac{\operatorname{Tr}\left(F_{x}, K_{\bar{x}}\right)}{\# \operatorname{Aut}_{x}\left(\mathbb{F}_{q}\right)}=: c_{1}\left(\mathscr{X}_{0}, K_{0}\right) .
\end{aligned}
$$

Here the second equality follows from [Laszlo and Olsson 2008b, 12.5.3]. Thus we reduce to the case when $\mathscr{Y}_{0}=\operatorname{Spec} \mathbb{F}_{q}$.

If $K_{0}^{\prime} \rightarrow K_{0} \rightarrow K_{0}^{\prime \prime} \rightarrow K_{0}^{\prime}[1]$ is an exact triangle of convergent complexes in $W_{m}^{-}$,stra $\left(\mathscr{Q}_{0}, \overline{\mathbb{Q}}_{\ell}\right)$, then by Corollary 4.4 and Theorem 4.2 (i) we have

$$
\begin{aligned}
c_{1}\left(\mathscr{X}_{0}, K_{0}\right) & =c_{1}\left(\mathscr{X}_{0}, K_{0}^{\prime}\right)+c_{1}\left(\mathscr{Q}_{0}, K_{0}^{\prime \prime}\right) \quad \text { and } \\
c_{1}\left(\mathscr{Y}_{0}, f_{!} K_{0}\right) & =c_{1}\left(\mathscr{Y}_{0}, f_{!} K_{0}^{\prime}\right)+c_{1}\left(\mathscr{Y}_{0}, f_{!} K_{0}^{\prime \prime}\right) .
\end{aligned}
$$

If $j: \mathscr{U}_{0} \rightarrow \mathscr{L}_{0}$ is an open substack with complement $i: \mathscr{L}_{0} \rightarrow \mathscr{X}_{0}$, then

$$
c_{1}\left(\mathscr{L}_{0}, j_{!} j^{*} K_{0}\right)=c_{1}\left(\mathscr{U}_{0}, j^{*} K_{0}\right) \quad \text { and } c_{1}\left(\mathscr{X}_{0}, i_{*} i^{*} K_{0}\right)=c_{1}\left(\mathscr{L}_{0}, i^{*} K_{0}\right) .
$$

By noetherian induction we can shrink $\mathscr{L}_{0}$ to a nonempty open substack. So we may assume the inertia stack $\mathscr{I}_{0}$ is flat over $\mathscr{L}_{0}$, with rigidification $\pi: \mathscr{X}_{0} \rightarrow X_{0}$, where $X_{0}$ is a scheme. If Theorem 4.2 (ii) holds for two composable morphisms $f$ and $g$, then it holds for $g \circ f$. So we reduce to two cases as before: $\mathscr{X}_{0}=X_{0}$ 
is a scheme, or $\mathscr{L}_{0}=B G_{0}$, where $G_{0}$ is either a connected algebraic group, or a finite étale algebraic group over $\mathbb{F}_{q}$. We may assume $X_{0}$ is separated, by further shrinking (for instance to an affine open subscheme).

For a complex of sheaves $K_{0}$ and an integer $n$, we have an exact triangle

$$
\tau_{<n} K_{0} \longrightarrow \tau_{<n+1} K_{0} \longrightarrow \mathscr{H}^{n}\left(K_{0}\right)[-n] \longrightarrow,
$$

so

$$
\begin{aligned}
c_{1}\left(\tau_{<n+1} K_{0}\right) & =c_{1}\left(\tau_{<n} K_{0}\right)+c_{1}\left(\mathscr{H}^{n}\left(K_{0}\right)[-n]\right) \\
& =c_{1}\left(\tau_{<n} K_{0}\right)+(-1)^{n} c_{1}\left(\mathscr{H}^{n}\left(K_{0}\right)\right) .
\end{aligned}
$$

Since $K_{0}$ is bounded above, $\tau_{<N} K_{0} \simeq K_{0}$ for $N \gg 0$. Since $K_{0}$ is convergent, $c_{1}\left(\tau_{<n} K_{0}\right) \rightarrow 0$ absolutely as $n \rightarrow-\infty$, so the series $\sum_{n \in \mathbb{Z}}(-1)^{n} c_{1}\left(\mathscr{H}^{n}\left(K_{0}\right)\right)$ converges absolutely to $c_{1}\left(K_{0}\right)$.

Applying $R \Gamma_{c}$ we get an exact triangle

$$
R \Gamma_{c}\left(\mathscr{X}_{0}, \tau_{<n} K_{0}\right) \longrightarrow R \Gamma_{c}\left(\mathscr{X}_{0}, \tau_{<n+1} K_{0}\right) \longrightarrow R \Gamma_{c}\left(\mathscr{X}_{0}, \mathscr{H}^{n} K_{0}\right)[-n] \longrightarrow
$$

in $W^{-}\left(\operatorname{Spec} \mathbb{F}_{q}, \overline{\mathbb{Q}}_{\ell}\right)$. We claim that, for $\mathscr{X}_{0}=X_{0}$ a scheme, or $B G_{0}$, we have

$$
\lim _{n \rightarrow-\infty} c_{1}\left(\operatorname{Spec} \mathbb{F}_{q}, R \Gamma_{c}\left(\mathscr{L}_{0}, \tau_{<n} K_{0}\right)\right)=0
$$

absolutely. Recall that $c_{1}\left(R \Gamma_{c}\left(\tau_{<n} K_{0}\right)\right)=\sum_{i \in \mathbb{Z}}(-1)^{i} \iota \operatorname{Tr}\left(F, H_{c}^{i}\left(\mathscr{X}, \tau_{<n} K\right)\right)$, so we need to show that

$$
\sum_{i \in \mathbb{Z}} \sum_{H_{c}^{i}\left(\mathscr{R}, \tau_{<n} K\right), F}|\alpha| \rightarrow 0 \quad \text { as } n \rightarrow-\infty .
$$

From the spectral sequence

$$
H_{c}^{r}\left(\mathscr{X}, \mathscr{H}^{k} \tau_{<n} K\right) \Longrightarrow H_{c}^{r+k}\left(\mathscr{X}, \tau_{<n} K\right)
$$

we see that

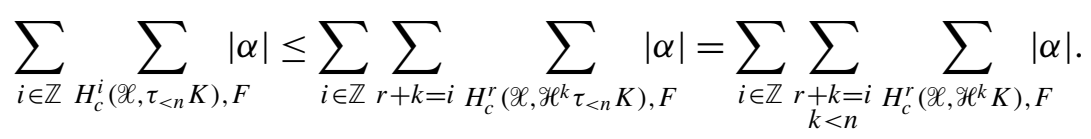

Let $d=\operatorname{dim} \mathscr{X}_{0}$ (see 9.1). In the cases where $\mathscr{X}_{0}$ is a scheme or $B G_{0}$, we have $H_{c}^{r}(\mathscr{X}, \mathscr{F})=0$ for every sheaf $\mathscr{F}$ unless $r \leq 2 d$ (see 4.8.2 and Lemma 4.6). Therefore

$$
\sum_{i \in \mathbb{Z}} \sum_{\substack{r+k=i \\ k<n}} \sum_{H_{c}^{r}\left(\mathscr{Q}, \mathscr{H}^{k} K\right), F}|\alpha| \leq \sum_{i<n+2 d} \sum_{r+k=i} \sum_{H_{c}^{r}\left(\mathscr{Q}, \mathscr{H}^{k} K\right), F}|\alpha|,
$$

and it suffices to show that the series

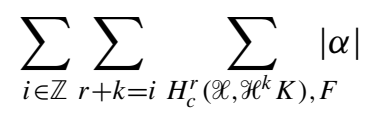


converges. We already proved this for $B G_{0}$ in Lemma 4.9, and for schemes $X_{0}$ in Lemma 4.10 (we may shrink $X_{0}$ so that the assumption in Lemma 4.10 is satisfied).

Note that in the two cases $\mathscr{X}_{0}=X_{0}$ or $B G_{0}$, Theorem 4.2 (ii) holds when $K_{0}$ is a sheaf concentrated in degree 0 . For separated schemes $X_{0}$, this is a classical result of Grothendieck [1965] and Verdier [1967]; for $B G_{0}$, this is done in Propositions 5.1 and 5.2. Therefore, for a general convergent complex $K_{0}$, we have

$$
\begin{aligned}
c_{1}\left(R \Gamma_{c}\left(\tau_{<n+1} K_{0}\right)\right) & =c_{1}\left(R \Gamma_{c}\left(\tau_{<n} K_{0}\right)\right)+c_{1}\left(R \Gamma_{c}\left(\mathscr{H}^{n} K_{0}\right)[-n]\right) \\
& =c_{1}\left(R \Gamma_{c}\left(\tau_{<n} K_{0}\right)\right)+(-1)^{n} c_{1}\left(\mathscr{H}^{n} K_{0}\right),
\end{aligned}
$$

and so

$$
c_{1}\left(R \Gamma_{c}\left(K_{0}\right)\right)=\sum_{n \in \mathbb{Z}}(-1)^{n} c_{1}\left(\mathscr{H}^{n} K_{0}\right)+\lim _{n \rightarrow-\infty} c_{1}\left(R \Gamma_{c}\left(\tau_{<n} K_{0}\right)\right)=c_{1}\left(K_{0}\right) .
$$

Corollary 5.3. Let $f: \mathscr{X}_{0} \rightarrow \mathscr{Y}_{0}$ be a morphism of $\mathbb{F}_{q}$-algebraic stacks, and let $K_{0} \in W_{m}^{- \text {,stra }}\left(\mathscr{X}_{0}, \overline{\mathbb{Q}}_{\ell}\right)$ be a convergent complex of sheaves. Then

$$
L\left(\mathscr{X}_{0}, K_{0}, t\right)=L\left(\mathscr{Y}_{0}, f_{!} K_{0}, t\right) .
$$

\section{Infinite products}

For a convergent complex $K_{0}$ on $\mathscr{X}_{0}$, the series $\sum_{v \geq 1} c_{v}\left(K_{0}\right) t^{v} / v$ (and hence the $L$-series $\left.L\left(\mathscr{X}_{0}, K_{0}, t\right)\right)$ usually has a finite radius of convergence. For instance, we have the following lemma.

Lemma 6.1. Let $X_{0} / \mathbb{F}_{q}$ be a variety of dimension $d$. Then the radius of convergence of $\sum_{v \geq 1} c_{v}\left(X_{0}\right) t^{v} / v$ is $1 / q^{d}$.

Proof. Let $f_{X_{0}}(t)=\sum_{v \geq 1} c_{v}\left(X_{0}\right) t^{v} / v$. Let $Y_{0}$ be an irreducible component of $X_{0}$ with complement $U_{0}$. Then $c_{v}\left(X_{0}\right)=c_{v}\left(Y_{0}\right)+c_{v}\left(U_{0}\right)$, and since all the $c_{v}$-terms are nonnegative, we see that the radius of convergence of $f_{X_{0}}(t)$ is the minimum of that of $f_{Y_{0}}(t)$ and that of $f_{U_{0}}(t)$. Since $\max \left\{\operatorname{dim}\left(Y_{0}\right), \operatorname{dim}\left(U_{0}\right)\right\}=d$, and $U_{0}$ has fewer irreducible component than $X_{0}$, by induction we can assume $X_{0}$ is irreducible.

Then there exists an open dense subscheme $U_{0} \subset X_{0}$ that is smooth over Spec $\mathbb{F}_{q}$. Let $Z_{0}=X_{0}-U_{0}$, then $\operatorname{dim}\left(Z_{0}\right)<\operatorname{dim}\left(X_{0}\right)=d$. From the cohomology sequence

$$
H_{c}^{2 d-1}(Z) \longrightarrow H_{c}^{2 d}(U) \longrightarrow H_{c}^{2 d}(X) \longrightarrow H_{c}^{2 d}(Z)
$$

we see that $H_{c}^{2 d}(X)=H_{c}^{2 d}(U)=\overline{\mathbb{Q}}_{\ell}(-d)$. The Frobenius eigenvalues $\left\{\alpha_{i j}\right\}_{j}$ on $H_{c}^{i}(X)$ have $\iota$-weights $\leq i$, for $0 \leq i<2 d$ [Deligne 1980, 3.3.4]. By the fixed point 
formula,

$$
\begin{aligned}
\frac{c_{v}\left(X_{0}\right)}{c_{v+1}\left(X_{0}\right)}=\frac{q^{v d}+\sum_{0 \leq i<2 d}(-1)^{i} \sum_{j} \alpha_{i j}^{v}}{q^{(v+1) d}+\sum_{0 \leq i<2 d}(-1)^{i} \sum_{j} \alpha_{i j}^{v+1}} & \\
& =\frac{\frac{1}{q^{d}}+\frac{1}{q^{d}} \sum_{0 \leq i<2 d}(-1)^{i} \sum_{j}\left(\frac{\alpha_{i j}}{q^{d}}\right)^{v}}{1+\sum_{0 \leq i<2 d}(-1)^{i} \sum_{j}\left(\frac{\alpha_{i j}}{q^{d}}\right)^{v+1}}
\end{aligned}
$$

which converges to $1 / q^{d}$ as $v \rightarrow \infty$, therefore the radius of convergence of $f_{X_{0}}(t)$ is

$$
\lim _{v \rightarrow \infty} \frac{c_{v}\left(X_{0}\right) / v}{c_{v+1}\left(X_{0}\right) /(v+1)}=\frac{1}{q^{d}} .
$$

In order to prove the meromorphic continuation of Theorem 8.1, we want to express the $L$-series as a possibly infinite product. For schemes, if we consider only bounded complexes, the $L$-series can be expressed as a finite alternating product of polynomials $P_{n}\left(X_{0}, K_{0}, t\right)$, so it is rational [Grothendieck 1965]. In the stack case, even for the sheaf $\overline{\mathbb{Q}}_{\ell}$, there might be infinitely many nonzero compact cohomology groups, and we need to consider the issue of convergence of the coefficients in an infinite products.

Definition 6.2. Let $f_{n}(t)=\sum_{k \geq 0} a_{n k} t^{k} \in \mathbb{C}[[t]]$ be a sequence of power series over $\mathbb{C}$. The sequence is said to be convergent term by term, if for each $k$, the sequence $\left(a_{n k}\right)_{n}$ converges, and the series

$$
\lim _{n \rightarrow \infty} f_{n}(t):=\sum_{k \geq 0} t^{k} \lim _{n \rightarrow \infty} a_{n k}
$$

is called the limit of the sequence $\left(f_{n}(t)\right)_{n}$.

6.2.1. Strictly speaking, a series (respectively infinite product) is defined to be a sequence $\left(a_{n}\right)_{n}$, usually written as an "infinite sum" (respectively "infinite product") so that $\left(a_{n}\right)_{n}$ is the sequence of finite partial sums (respectively finite partial products) of it. So the definition above applies to series and infinite products.

Recall that $\log (1+g)=\sum_{m \geq 1}(-1)^{m+1} g^{m} / m$ for $g \in t \mathbb{C}[[t]$.

Lemma 6.3. (i) Let $f_{n}(t)=1+\sum_{k \geq 1} a_{n k} t^{k} \in \mathbb{C}[[t]]$ be a sequence of power series. Then $\left(f_{n}(t)\right)_{n}$ is convergent term by term if and only if $\left(\log f_{n}(t)\right)_{n}$ is convergent term by term, and

$$
\lim _{n \rightarrow \infty} \log f_{n}(t)=\log \lim _{n \rightarrow \infty} f_{n}(t) .
$$

(ii) Let $f$ and $g$ be two power series with constant term 1 . Then

$$
\log (f g)=\log (f)+\log (g) \text {. }
$$


(iii) Let $\left.f_{n}(t) \in 1+t \mathbb{C}[t]\right]$ be a sequence as in (i). Then the infinite product $\prod_{n \geq 1} f_{n}(t)$ converges term by term if and only if the series $\sum_{n \geq 1} \log f_{n}(t)$ converges term by term, and

$$
\sum_{n \geq 1} \log f_{n}(t)=\log \prod_{n \geq 1} f_{n}(t)
$$

Proof. (i) We have

$$
\begin{aligned}
\log f_{n}(t)=\sum_{m \geq 1}(-1)^{m+1}( & \left.\sum_{k \geq 1} a_{n k} t^{k}\right)^{m} / m \\
=t \cdot a_{n 1}+ & t^{2}\left(a_{n 2}-\frac{1}{2} a_{n 1}^{2}\right)+t^{3}\left(a_{n 3}-a_{n 1} a_{n 2}+\frac{1}{3} a_{n 1}^{3}\right) \\
& +t^{4}\left(a_{n 4}-a_{n 1} a_{n 3}-\frac{1}{2} a_{n 2}^{2}+a_{n 1}^{2} a_{n 2}\right)+\cdots=: \sum_{k \geq 1} A_{n k} t^{k} .
\end{aligned}
$$

In particular, for each $k$, the function $A_{n k}-a_{n k}=h\left(a_{n 1}, \ldots, a_{n, k-1}\right)$ is a polynomial in $a_{n 1}, \ldots, a_{n, k-1}$ with rational coefficients. So if $\left(a_{n k}\right)_{n}$ converges for each $k$, then $\left(A_{n k}\right)_{n}$ also converges, and by induction the converse also holds. If we have $\lim _{n \rightarrow \infty} a_{n k}=a_{k}$, then $\lim _{n \rightarrow \infty} A_{n k}=a_{k}+h\left(a_{1}, \ldots, a_{k-1}\right)$, and

$\log \lim _{n \rightarrow \infty} f_{n}(t)=\log \left(1+\sum_{k \geq 1} a_{k} t^{k}\right)=\sum_{k \geq 1}\left(a_{k}+h\left(a_{1}, \ldots, a_{k-1}\right)\right) t^{k}=\lim _{n \rightarrow \infty} \log f_{n}(t)$.

(ii) $\log$ and exp are inverse to each other on power series, so it suffices to prove that for $f$ and $g \in t \mathbb{C} \llbracket t \rrbracket$, we have

$$
\exp (f+g)=\exp (f) \exp (g)
$$

This follows from the binomial formula:

$$
\begin{array}{r}
\exp (f+g)=\sum_{n \geq 0}(f+g)^{n} / n !=\sum_{n \geq 0} \frac{1}{n !} \sum_{k=0}^{n}\left(\begin{array}{l}
n \\
k
\end{array}\right) f^{k} g^{n-k}=\sum_{n \geq 0} \sum_{k=0}^{n} \frac{f^{k}}{k !} \cdot \frac{g^{n-k}}{(n-k) !} \\
=\sum_{i, j \geq 0} \frac{f^{i}}{i !} \cdot \frac{g^{j}}{j !}=\left(\sum_{i \geq 0} f^{i} / i !\right)\left(\sum_{j \geq 0} g^{j} / j !\right)=\exp (f) \exp (g) .
\end{array}
$$

(iii) Let $F_{N}(t)=\prod_{n=1}^{N} f_{n}(t)$. Applying (i) to the sequence $\left(F_{N}(t)\right)_{N}$, we see that the infinite product $\prod_{n \geq 1} f_{n}(t)$ converges term by term if and only if (by definition) $\left(F_{N}(t)\right)_{N}$ converges term by term, if and only if the sequence $\left(\log F_{N}(t)\right)_{N}$ converges term by term, if and only if (by definition) the series $\sum_{n \geq 1} \log f_{n}(t)$ converges term by term, since by (ii)

$$
\log \prod_{n=1}^{N} f_{n}(t)=\sum_{n=1}^{N} \log f_{n}(t)
$$


Also

$$
\begin{aligned}
\log \prod_{n \geq 1} f_{n}(t)=\log \lim _{N \rightarrow \infty} F_{N}(t)=\lim _{N \rightarrow \infty} & \log F_{N}(t) \\
& =\lim _{N \rightarrow \infty} \sum_{n=1}^{N} \log f_{n}(t)=: \sum_{n \geq 1} \log f_{n}(t) .
\end{aligned}
$$

6.4. For a complex of sheaves $K_{0}$ on $\mathscr{Q}_{0}$ and $n \in \mathbb{Z}$, define

$$
P_{n}\left(\mathscr{Q}_{0}, K_{0}, t\right):=\operatorname{det}\left(1-F t, H_{c}^{n}(\mathscr{X}, K)\right) .
$$

We regard $P_{n}\left(\mathscr{Q}_{0}, K_{0}, t\right)^{ \pm 1}$ as a complex power series with constant term 1 via $\iota$.

Proposition 6.5. For every convergent complex of sheaves $K_{0} \in W_{m}^{- \text {,stra }}\left(\mathscr{Q}_{0}, \overline{\mathbb{Q}}_{\ell}\right)$, the infinite product

$$
\prod_{n \in \mathbb{Z}} P_{n}\left(\mathscr{Q}_{0}, K_{0}, t\right)^{(-1)^{n+1}}
$$

is convergent term by term to the $L$-series $L\left(\mathscr{L}_{0}, K_{0}, t\right)$.

Proof. The complex $R \Gamma_{c}(\mathscr{X}, K)$ is bounded above, so $P_{n}\left(\mathscr{X}_{0}, K_{0}, t\right)=1$ for $n \gg 0$, and the infinite product is a one-direction limit, namely $n \rightarrow-\infty$.

Let $\alpha_{n 1}, \ldots, \alpha_{n m_{n}}$ be the eigenvalues (counted with multiplicity) of the morphism $F$ on $H_{c}^{n}(\mathscr{X}, K)$, regarded as complex numbers via $\iota$, so that

$$
P_{n}(t)=P_{n}\left(\mathscr{X}_{0}, K_{0}, t\right)=\left(1-\alpha_{n 1} t\right) \cdots\left(1-\alpha_{n m_{n}} t\right) .
$$

By Lemma 6.3 (iii) it suffices to show that the series

$$
\sum_{n \in \mathbb{Z}}(-1)^{n+1} \log P_{n}(t)
$$

converges term by term to $\sum_{v \geq 1} c_{v}\left(K_{0}\right) t^{v} / v$.

We have

$$
\begin{aligned}
\sum_{n \in \mathbb{Z}}(-1)^{n+1} \log P_{n}(t)=\sum_{n \in \mathbb{Z}}(-1)^{n+1} \log \prod_{i}\left(1-\alpha_{n i} t\right) \\
=\sum_{n \in \mathbb{Z}}(-1)^{n} \sum_{i} \sum_{v \geq 1} \frac{\alpha_{n i}^{v} t^{v}}{v}=\sum_{v \geq 1} \frac{t^{v}}{v} \sum_{n \in \mathbb{Z}}(-1)^{n} \sum_{i} \alpha_{n i}^{v}=\sum_{v \geq 1} \frac{t^{v}}{v} c_{v}\left(R \Gamma_{c}\left(K_{0}\right)\right),
\end{aligned}
$$

which converges term by term by Theorem 4.2 (i), and is equal to $\sum_{v \geq 1} c_{v}\left(K_{0}\right) t^{v} / v$ by Theorem 4.2 (ii).

Remark 6.5.1. In particular we have

$$
Z\left(\mathscr{X}_{0}, t\right)=\prod_{n \in \mathbb{Z}} P_{n}\left(\mathscr{X}_{0}, t\right)^{(-1)^{n+1}}
$$


where $P_{n}\left(\mathscr{C}_{0}, t\right)=P_{n}\left(\mathscr{X}_{0}, \overline{\mathbb{Q}}_{\ell}, t\right)$. This generalizes the classical result for schemes [Grothendieck 1965, 5.1]. When we want to emphasize the dependence on the prime $\ell$, we will write $P_{n, \ell}\left(\mathscr{Q}_{0}, t\right)$.

If $G_{0}$ is a connected $\mathbb{F}_{q}$-algebraic group, 4.8.2 shows that the zeta function of $B G_{0}$ is given by

$$
Z\left(B G_{0}, t\right)=\prod_{r \geq 0} \prod_{\substack{m_{i j} \geq 0 \\ \sum_{i, j} m_{i j}(i+1)=2 r}}\left(1-q^{-d} \prod_{i, j} \alpha_{i j}^{-m_{i j}} \cdot t\right)^{-1}=\prod_{m_{i j} \geq 0}\left(1-q^{-d} \prod_{i, j} \alpha_{i j}^{-m_{i j}} \cdot t\right)^{-1} .
$$

\section{Examples of zeta functions}

In this section we compute the zeta functions of some stacks, and in each example we do it in two ways: by counting rational points and by computing cohomology groups. Also we investigate some analytic properties.

Example 7.1. $B \mathbb{G}_{m}$. By 4.7 (iii) we have $c_{v}\left(B \mathbb{G}_{m}\right)=1 / c_{v}\left(\mathbb{G}_{m}\right)$, so the zeta function is

$$
Z\left(B \mathbb{G}_{m}, t\right)=\exp \left(\sum_{v \geq 1} c_{v}\left(B \mathbb{G}_{m}\right) \frac{t^{v}}{v}\right)=\exp \left(\sum_{v \geq 1} \frac{1}{q^{v}-1} \frac{t^{v}}{v}\right) .
$$

Using Borel's theorem 4.8 one can show (or see [Laumon and Moret-Bailly 2000, 19.3.2]) that the cohomology $\operatorname{ring} H^{*}\left(B \mathbb{G}_{m}\right)$ is a polynomial ring $\overline{\mathbb{Q}}_{\ell}[T]$, generated by a variable $T$ of degree 2, and that the Frobenius action on cohomology is given by $F T^{n}=q^{n} T^{n}$. So by Poincaré duality, we have

$$
\begin{aligned}
\operatorname{Tr}\left(F, H_{c}^{-2 n-2}\left(B \mathbb{G}_{m}\right)\right)=\operatorname{Tr}\left(F, H_{c}^{-2 n-2}\left(B \mathbb{G}_{m},\right.\right. & \left.\left.\overline{\mathbb{Q}}_{\ell}(-1)\right)\right) / q \\
& =\operatorname{Tr}\left(F^{-1}, H^{2 n}\left(B \mathbb{G}_{m}\right)\right) / q=q^{-n-1} .
\end{aligned}
$$

This gives

$$
\prod_{n \in \mathbb{Z}} P_{n}\left(B \mathbb{G}_{m}, t\right)^{(-1)^{n+1}}=\prod_{n \geq 1}\left(1-q^{-n} t\right)^{-1} .
$$

It is easy to verify the result in Remark 6.5.1 directly:

$$
\begin{array}{r}
\exp \left(\sum_{v \geq 1} \frac{1}{q^{v}-1} \frac{t^{v}}{v}\right)=\exp \left(\sum_{v \geq 1} \frac{1 / q^{v}}{1-1 / q^{v}} \frac{t^{v}}{v}\right)=\exp \left(\sum_{v \geq 1} \frac{t^{v}}{v} \sum_{n \geq 1} \frac{1}{q^{n v}}\right) \\
=\prod_{n \geq 1} \exp \left(\sum_{v \geq 1} \frac{\left(t / q^{n}\right)^{v}}{v}\right)=\prod_{n \geq 1}\left(1-t / q^{n}\right)^{-1} .
\end{array}
$$

There is also a functional equation

$$
Z\left(B \mathbb{G}_{m}, q t\right)=\frac{1}{1-t} Z\left(B \mathbb{G}_{m}, t\right),
$$


which implies that $Z\left(B \mathbb{G}_{m}, t\right)$ has a meromorphic continuation to the whole complex plane, with simple poles at $t=q^{n}$, for $n \geq 1$.

$H_{c}^{-2 n-2}\left(B \mathbb{G}_{m}\right)$ is pure of weight $-2 n-2$. A natural question is if Deligne's theorem of weights [Deligne 1980, 3.3.4] still holds for algebraic stacks. Olsson told me that it does not hold in general, as the following example shows.

Example 7.2. $B E$, where $E$ is an elliptic curve over $\mathbb{F}_{q}$. Again by 4.7 (iii) we have

$$
c_{v}(B E)=1 / \# E\left(\mathbb{F}_{q^{v}}\right) .
$$

Let $\alpha$ and $\beta$ be the roots of the reciprocal characteristic polynomial of the Frobenius on $H^{1}(E)$ :

$$
x^{2}-\left(1+q-c_{1}(E)\right) x+q=0 .
$$

Then for every $v \geq 1$, we have $c_{v}(E)=1-\alpha^{v}-\beta^{v}+q^{v}=\left(1-\alpha^{v}\right)\left(1-\beta^{v}\right)$. So $c_{v}(B E)=\frac{1}{\left(1-\alpha^{v}\right)\left(1-\beta^{v}\right)}=\frac{\alpha^{-v}}{1-\alpha^{-v}} \cdot \frac{\beta^{-v}}{1-\beta^{-v}}$

$$
=\left(\sum_{n \geq 1} \alpha^{-n v}\right)\left(\sum_{m \geq 1} \beta^{-n v}\right)=\sum_{n, m \geq 1}\left(\frac{1}{\alpha^{n} \beta^{m}}\right)^{v},
$$

and the zeta function is

$$
\begin{aligned}
Z(B E, t)=\exp \left(\sum_{v \geq 1} c_{v}(B E) \frac{t^{v}}{v}\right) & =\exp \left(\sum_{\substack{n, m \geq 1 \\
v \geq 1}}\left(\frac{t}{\alpha^{n} \beta^{m}}\right)^{v} / v\right)=\prod_{n, m \geq 1}\left(1-\frac{t}{\alpha^{n} \beta^{m}}\right)^{-1} .
\end{aligned}
$$

To compute its cohomology, one can apply Borel's theorem 4.8 to $E$, and we have $N=N^{1}=H^{1}(E)$, so $N[-1]$ is a 2-dimensional vector space sitting in degree 2 , on which $F$ has eigenvalues $\alpha$ and $\beta$. Then $H^{*}(B E)$ is a polynomial ring $\overline{\mathbb{Q}}_{\ell}[a, b]$ in two variables, both sitting in degree 2 , and the basis $a, b$ can be chosen so that the Frobenius action $F$ on $H^{2}(B E)$ is upper triangular (or even diagonal)

$$
\left[\begin{array}{ll}
\alpha & \gamma \\
& \beta
\end{array}\right] \text {. }
$$

Then $F$ acting on

$$
H^{2 n}(B E)=\operatorname{Sym}^{n} N[-1]=\overline{\mathbb{Q}}_{\ell}\left\langle a^{n}, a^{n-1} b, \ldots, b^{n}\right\rangle
$$

can be represented by

$$
\left[\begin{array}{cccc}
\alpha^{n} & * & * & * \\
& \alpha^{n-1} \beta & * & * \\
& & \ddots & * \\
& & & \beta^{n}
\end{array}\right],
$$


with eigenvalues $\alpha^{n}, \alpha^{n-1} \beta, \ldots, \beta^{n}$. So the eigenvalues of $F$ on $H_{c}^{-2-2 n}(B E)$ are $q^{-1} \alpha^{-n}, q^{-1} \alpha^{1-n} \beta^{-1}, \ldots, q^{-1} \beta^{-n}$ and

$$
\begin{aligned}
\prod_{n \in \mathbb{Z}} P_{n}(B E, t)^{(-1)^{n+1}=1 /} & \left(1-q^{-1} t\right) \cdot\left[\left(1-q^{-1} \alpha^{-1} t\right)\left(1-q^{-1} \beta^{-1} t\right)\right] \\
\cdot & {\left[\left(1-q^{-1} \alpha^{-2} t\right)\left(1-q^{-1} \alpha^{-1} \beta^{-1} t\right)\left(1-q^{-1} \beta^{-2} t\right)\right] \cdots . }
\end{aligned}
$$

Note that the right hand side is the same as $Z(B E, t)$ above (since $\alpha \beta=q$ ).

Let $Z_{1}(t):=Z(B E, q t)$. Its radius of convergence is 1 , since by Lemma 6.1

$$
\lim _{v \rightarrow \infty} \frac{c_{v}(B E)}{c_{v+1}(B E)}=\lim _{v \rightarrow \infty} \frac{c_{v+1}(E)}{c_{v}(E)}=q .
$$

There is also a functional equation

$$
Z_{1}(\alpha t)=\frac{1}{1-\alpha t} Z_{1}(t) Z_{2}(t)
$$

where

$$
Z_{2}(t)=\frac{1}{\left(1-\alpha \beta^{-1} t\right)\left(1-\alpha \beta^{-2} t\right)\left(1-\alpha \beta^{-3} t\right) \cdots} .
$$

$Z_{2}(t)$ is holomorphic in the open unit disk and satisfies the functional equation

$$
Z_{2}(\beta t)=\frac{1}{1-\alpha t} Z_{2}(t)
$$

Therefore $Z_{2}(t)$, and hence $Z(B E, t)$, has a meromorphic continuation to the whole complex $t$-plane with the obvious poles.

Remark 7.2.1. $H_{c}^{-2-2 n}(B E)$ is pure of weight $-2-n$, which is not $\leq-2-2 n$ unless $n=0$. So the upper bound of weights for schemes fails for $B E$. This also leads to the failure of the decomposition theorem for $B E$; see [Sun 2012, §1], for the example of a pure complex on $B E$ which is not geometrically semisimple.

Also note that, the Equation (7.2.1) is independent of $\ell$, so the polynomials $P_{n, \ell}(B E, t)$ are independent of $\ell$.

Example 7.3. $B G_{0}$, where $G_{0}$ is a finite étale $\mathbb{F}_{q}$-group scheme, corresponding to a finite group $G$ and a Frobenius automorphism $\sigma$ on it. Then $B G_{0}\left(\mathbb{F}_{q^{v}}\right) \simeq G / \rho^{(v)}$, where $\rho^{(v)}$ is the right action of $G$ on the set $G$ given by $h: g \mapsto \sigma^{v}\left(h^{-1}\right) g h$. So

$$
c_{v}\left(B G_{0}\right)=\sum_{[g] \in G / \rho^{(v)}} \frac{1}{\# \operatorname{Stab}_{\rho^{(v)}}(g)}=\frac{\# G}{\# G}=1,
$$

and the zeta function is

$$
Z\left(B G_{0}, t\right)=\frac{1}{1-t} .
$$

Its cohomology groups are given in Lemma 4.6: $H_{c}^{0}(B G)=\overline{\mathbb{Q}}_{\ell}$, and other $H_{c}^{i}=0$. This verifies Remark 6.5.1. 
Note that $Z\left(B G_{0}, t\right)$ is the same as the zeta function of its coarse moduli space Spec $\mathbb{F}_{q}$. As a consequence, for every $\mathbb{F}_{q}$-algebraic stack $\mathscr{X}_{0}$, with finite inertia $\mathscr{I}_{0} \rightarrow \mathscr{X}_{0}$ and coarse moduli space $\pi: \mathscr{L}_{0} \rightarrow X_{0}$ [Conrad 2005, 1.1], we have $Z\left(\mathscr{Q}_{0}, t\right)=Z\left(X_{0}, t\right)$, and hence it is a rational function. This is because for every $x \in X_{0}\left(\mathbb{F}_{q^{v}}\right)$, the fiber $\pi^{-1}(x)$ is a neutral gerbe over Spec $k(x)$, and from the above we see that $c_{v}\left(\pi^{-1}(x)\right)=1$, and hence $c_{v}\left(\mathscr{Q}_{0}\right)=c_{v}\left(X_{0}\right)$. The fact that $Z\left(X_{0}, t\right)$ is a rational function follows from [Knutson 1971, II, 6.7] and noetherian induction. More generally, we have the following.

Proposition 7.3.1. Let $\mathscr{X}_{0}$ be an $\mathbb{F}_{q}$-algebraic stack. Suppose that $\mathscr{X}_{0}$ either has finite inertia, or is Deligne-Mumford (not necessarily separated). Then for every $K_{0} \in W^{b}\left(\mathscr{X}_{0}, \overline{\mathbb{Q}}_{\ell}\right)$, the L-series $L\left(\mathscr{Q}_{0}, K_{0}, t\right)$ is a rational function.

Proof. It suffices to show that Theorem 4.2 holds for the structural morphism $\mathscr{Q}_{0} \rightarrow$ Spec $\mathbb{F}_{q}$ and $K_{0} \in W^{b}\left(\mathscr{C}_{0}, \overline{\mathbb{Q}}_{\ell}\right)$ in these two cases. We will not make explicit use of the fact from Remark 2.8.1 that $K_{0}$ is $\iota$-mixed.

Case when $\mathscr{X}_{0}$ has finite inertia. Let $\pi: \mathscr{C}_{0} \rightarrow X_{0}$ be its coarse moduli space. For any sheaf $\mathscr{F}_{0}$ on $\mathscr{X}_{0}$, we have isomorphisms $H_{c}^{r}\left(X, R^{0} \pi_{!} \mathscr{F}\right) \simeq H_{c}^{r}(\mathscr{X}, \mathscr{F})$ by Lemma 4.6, so $R \Gamma_{c}\left(\mathscr{X}_{0}, \mathscr{F}_{0}\right)$ is a bounded complex, hence a convergent complex. To prove the trace formula for $\mathscr{X}_{0} \rightarrow$ Spec $\mathbb{F}_{q}$ and the sheaf $\mathscr{F}_{0}$, it suffices to prove it for $\mathscr{X}_{0} \rightarrow X_{0}$ and $X_{0} \rightarrow \operatorname{Spec} \mathbb{F}_{q}$. The first case, when passing to fibers, is reduced to $B G_{0}$, and when passing to fibers again, it is reduced to the two subcases: when $G_{0}$ is finite, or when $G_{0}$ is connected. In both of these two cases as well as the case of an algebraic space $X_{0} \rightarrow$ Spec $\mathbb{F}_{q}$, the trace formula can be proved without using $\iota$-mixedness 5.2.1. Therefore, Theorem 4.2 holds for $\mathscr{X}_{0} \rightarrow \operatorname{Spec} \mathbb{F}_{q}$ and any sheaf, hence any bounded complex, on $\mathscr{X}_{0}$.

The trace formula is equivalent to the equality of power series

$$
L\left(\mathscr{X}_{0}, K_{0}, t\right)=\prod_{i \in \mathbb{Z}} P_{i}\left(\mathscr{X}_{0}, K_{0}, t\right)^{(-1)^{i+1}},
$$

and the right-hand side is a finite product, because $R \Gamma_{c}\left(\mathscr{L}_{0}, K_{0}\right)$ is bounded. Therefore, $L\left(\mathscr{Q}_{0}, K_{0}, t\right)$ is rational.

Case when $\mathscr{X}_{0}$ is Deligne-Mumford. For both (i) and (ii) of Theorem 4.2, we may replace $\mathscr{X}_{0}$ by a nonempty open substack, hence by [Laumon and Moret-Bailly $2000,6.1 .1]$ we may assume $\mathscr{X}_{0}$ is the quotient stack $\left[X_{0}^{\prime} / G\right]$, where $X_{0}^{\prime}$ is an affine $\mathbb{F}_{q}$-scheme of finite type and $G$ is a finite group acting on $X_{0}^{\prime}$. This stack has finite diagonal, and hence finite inertia, so by the previous case we are done. Also, we know that $R \Gamma_{c}\left(\mathscr{X}_{0}, K_{0}\right)$ is bounded, therefore $L\left(\mathscr{C}_{0}, K_{0}, t\right)$ is rational.

If one wants to use Poincaré duality to get a functional equation for the zeta function, [Olsson 2008a, 5.17] and [Laszlo and Olsson 2008b, 9.1.2] suggest that we should assume $\mathscr{L}_{0}$ to be proper smooth and of finite diagonal. Under these 
assumptions, one gets the expected functional equation for the zeta function, as well as the independence of $\ell$ for the coarse moduli space, which is proper but possibly singular. Examples of such stacks include the moduli stack of pointed stable curves $\bar{M}_{g, n}$ over $\mathbb{F}_{q}$.

Proposition 7.3.2. Let $\mathscr{X}_{0}$ be a proper smooth $\mathbb{F}_{q}$-algebraic stack of equidimension $d$, with finite diagonal, and let $\pi: \mathscr{X}_{0} \rightarrow X_{0}$ be its coarse moduli space. Then $Z\left(X_{0}, t\right)$ satisfies the usual functional equation

$$
Z\left(X_{0}, \frac{1}{q^{d} t}\right)= \pm q^{d \chi / 2} t^{\chi} Z\left(X_{0}, t\right),
$$

where $\chi:=\sum_{i=0}^{2 d}(-1)^{i} \operatorname{deg} P_{i, \ell}\left(X_{0}, t\right)$. Moreover, $H^{i}(X)$ is pure of weight $i$, for every $0 \leq i \leq 2 d$, and the reciprocal roots of each $P_{i, \ell}\left(X_{0}, t\right)$ are algebraic integers independent of $\ell$.

Proof. First we show that the adjunction map $\overline{\mathbb{Q}}_{\ell} \rightarrow \pi_{*} \pi^{*} \overline{\mathbb{Q}}_{\ell}=\pi_{*} \overline{\mathbb{Q}}_{\ell}$ is an isomorphism. Since $\pi$ is quasi-finite and proper [Conrad 2005, 1.1], we have $\pi_{*}=\pi$ ! [Olsson 2008a, 5.1] and $R^{r} \pi ! \overline{\mathbb{Q}}_{\ell}=0$ for $r \neq 0$ [Olsson 2008a, 5.8]. The natural map $\overline{\mathbb{Q}}_{\ell} \rightarrow R^{0} \pi_{*} \overline{\mathbb{Q}}_{\ell}$ is an isomorphism, since the geometric fibers of $\pi$ are connected.

Therefore $R \Gamma\left(\mathscr{Q}_{0}, \overline{\mathbb{Q}}_{\ell}\right)=R \Gamma\left(X_{0}, \pi_{*} \overline{\mathbb{Q}}_{\ell}\right)=R \Gamma\left(X_{0}, \overline{\mathbb{Q}}_{\ell}\right)$, and hence

$$
H^{i}(\mathscr{X}) \simeq H_{c}^{i}(\mathscr{X}) \simeq H^{i}(X) \simeq H_{c}^{i}(X)
$$

for all $i$ [Olsson 2008a, 5.17]. Let $P_{i}(t)=P_{i}\left(\mathscr{C}_{0}, t\right)=P_{i}\left(X_{0}, t\right)$. Since $X_{0}$ is an algebraic space of dimension $d, P_{i}(t)=1$ if $i \notin[0,2 d]$. Since $\mathscr{X}_{0}$ is proper and smooth, Poincaré duality gives a perfect pairing

$$
H^{i}(\mathscr{X}) \times H^{2 d-i}(\mathscr{X}) \longrightarrow \overline{\mathbb{Q}}_{\ell}(-d) .
$$

Following the standard proof for proper smooth varieties, see [Milne 2008, 27.12], we get the expected functional equation for $Z\left(\mathscr{X}_{0}, t\right)=Z\left(X_{0}, t\right)$.

$H^{i}(X)$ is mixed of weights $\leq i$ [Deligne 1980, 3.3.4], so by Poincaré duality, it is pure of weight $i$. Following the proof in [Deligne 1974a, p. 276)], this purity implies that the polynomials $P_{i, \ell}\left(X_{0}, t\right)$ have integer coefficients independent of $\ell$.

Remark 7.3.3. Weizhe Zheng suggested Proposition 7.3.1 to me. He also suggested that we give a functional equation relating $L\left(\mathscr{X}_{0}, D K_{0}, t\right)$ and $L\left(\mathscr{C}_{0}, K_{0}, t\right)$,

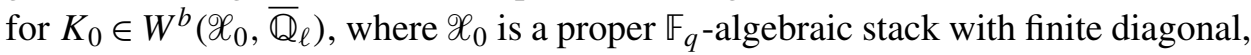
of equidimension $d$, but not necessarily smooth. Here is the functional equation:

$$
L\left(\mathscr{Q}_{0}, K_{0}, t^{-1}\right)=t^{\chi_{c}} \cdot Q \cdot L\left(\mathscr{X}_{0}, D K_{0}, t\right),
$$


where $\chi_{c}=\sum_{i=0}^{2 d}(-1)^{i} h_{c}^{i}(\mathscr{X}, K)$ and $Q=\left.\left(t^{\chi_{c}} L\left(\mathscr{X}_{0}, K_{0}, t\right)\right)\right|_{t=\infty}$. Note that the rational function $L\left(\mathscr{X}_{0}, K_{0}, t\right)$ has degree $-\chi_{c}$, hence $Q$ is well-defined. The proof is similar to the above.

Example 7.4. $B G L_{N}$. We have

$$
\# G L_{N}\left(\mathbb{F}_{q^{v}}\right)=\left(q^{v N}-1\right)\left(q^{v N}-q^{v}\right) \cdots\left(q^{v N}-q^{v(N-1)}\right),
$$

so one can use $c_{v}\left(B G L_{N}\right)=1 / c_{v}\left(G L_{N}\right)$ to compute $Z\left(B G L_{N}, t\right)$. One can also compute the cohomology groups of $B G L_{N}$ using Borel's theorem 4.8. We refer to [Behrend 1993, 2.3.2] for the result. Let us consider the case $N=2$ only. The general case is similar.

We have

$$
c_{v}\left(B G L_{2}\right)=\frac{1}{q^{4 v}}\left(1+\frac{1}{q^{v}}+\frac{2}{q^{2 v}}+\frac{2}{q^{3 v}}+\frac{3}{q^{4 v}}+\frac{3}{q^{5 v}}+\cdots\right),
$$

and therefore

$$
\begin{aligned}
Z\left(B G L_{2}, t\right)= & \exp \left(\sum_{v} \frac{\left(t / q^{4}\right)^{v}}{v}\right) \cdot \exp \left(\sum_{v} \frac{\left(t / q^{5}\right)^{v}}{v}\right) \cdot \exp \left(\sum_{v} \frac{2\left(t / q^{6}\right)^{v}}{v}\right) \cdots \\
& =\frac{1}{1-t / q^{4}} \cdot \frac{1}{1-t / q^{5}} \cdot\left(\frac{1}{1-t / q^{6}}\right)^{2} \cdot\left(\frac{1}{1-t / q^{7}}\right)^{2} \cdot\left(\frac{1}{1-t / q^{8}}\right)^{3} \cdots .
\end{aligned}
$$

So $Z\left(B G L_{2}, q t\right)=Z\left(B G L_{2}, t\right) \cdot Z_{1}(t)$, where

$$
Z_{1}(t)=\frac{1}{\left(1-t / q^{3}\right)\left(1-t / q^{5}\right)\left(1-t / q^{7}\right)\left(1-t / q^{9}\right) \cdots} .
$$

$Z_{1}(t)$ satisfies the functional equation

$$
Z_{1}\left(q^{2} t\right)=\frac{1}{1-t / q} \cdot Z_{1}(t)
$$

So $Z_{1}(t)$, and hence $Z\left(B G L_{2}, t\right)$, has a meromorphic continuation with the obvious poles.

The nonzero compactly supported cohomology groups of $B G L_{2}$ are given as follows:

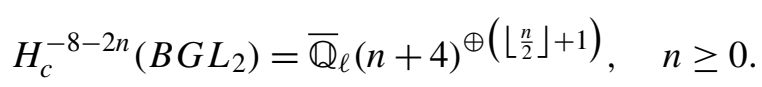

This gives

$$
\prod_{n \in \mathbb{Z}} P_{n}\left(B G L_{2}, t\right)^{(-1)^{n+1}}=\frac{1}{\left(1-t / q^{4}\right)\left(1-t / q^{5}\right)\left(1-t / q^{6}\right)^{2}\left(1-t / q^{7}\right)^{2} \cdots},
$$

and Remark 6.5.1 is verified. Note that the eigenvalues are $1 / q^{n+4}$, which are independent of $\ell$. 


\section{Analytic continuation}

We state and prove a generalized version of Theorem 1.3.

Theorem 8.1. Let $\mathscr{X}_{0}$ be an $\mathbb{F}_{q}$-algebraic stack, and let $K_{0} \in W_{m}^{- \text {,stra }}\left(\mathscr{Q}_{0}, \overline{\mathbb{Q}}_{\ell}\right)$ be a convergent complex. Then $L\left(\mathscr{X}_{0}, K_{0}, t\right)$ has a meromorphic continuation to the whole complex t-plane, and its poles can only be zeros of the polynomials $P_{2 n}\left(\mathscr{Q}_{0}, K_{0}, t\right)$ for some integers $n$.

We need a preliminary lemma. For an open subset $U \subset \mathbb{C}$, let $\mathcal{O}(U)$ be the set of analytic functions on $U$. There exists a sequence $\left\{K_{n}\right\}_{n \geq 1}$ of compact subsets of $U$ such that $U=\bigcup_{n} K_{n}$ and $K_{n} \subset\left(K_{n+1}\right)^{\circ}$. For $f$ and $g$ in $\mathcal{O}(U)$, define

$$
\rho_{n}(f, g)=\sup \left\{|f(z)-g(z)| ; z \in K_{n}\right\} \quad \text { and } \quad \rho(f, g)=\sum_{n=1}^{\infty}\left(\frac{1}{2}\right)^{n} \frac{\rho_{n}(f, g)}{1+\rho_{n}(f, g)} \text {. }
$$

Then $\rho$ is a metric on $O(U)$ and the topology it induces is independent of the subsets $\left\{K_{n}\right\}_{n}$ chosen (see [Conway 1973, VII, §1]).

The following lemma is from [Conway 1973, p. 167, 5.9].

Lemma 8.2. Let $U \subset \mathbb{C}$ be connected and open and let $\left(f_{n}\right)_{n}$ be a sequence in $\mathcal{O}(U)$ such that no $f_{n}$ is identically zero. If $\sum_{n}\left(f_{n}(z)-1\right)$ converges absolutely and uniformly on compact subsets of $U$, then $\prod_{n \geq 1} f_{n}(z)$ converges in $\mathcal{O}(U)$ to an analytic function $f(z)$. If $z_{0}$ is a zero of $f$, then $z_{0}$ is a zero of only a finite number of the functions $f_{n}$, and the multiplicity of the zero of $f$ at $z_{0}$ is the sum of the multiplicities of the zeros of the functions $f_{n}$ at $z_{0}$.

Now we prove Theorem 8.1.

Proof. Factorize $P_{n}\left(\mathscr{X}_{0}, K_{0}, t\right)$ as $\prod_{j=1}^{m_{n}}\left(1-\alpha_{n j} t\right)$ in $\mathbb{C}$. Since $R \Gamma_{c}\left(\mathscr{L}_{0}, K_{0}\right)$ is a convergent complex by Theorem $4.2(\mathrm{i})$, the series $\sum_{n, j}\left|\alpha_{n j}\right|$ converges.

By Proposition 6.5 we have

$$
L\left(\mathscr{L}_{0}, K_{0}, t\right)=\prod_{n \in \mathbb{Z}}\left(\prod_{j=1}^{m_{n}}\left(1-\alpha_{n j} t\right)\right)^{(-1)^{n+1}}
$$

as formal power series. Take $U$ to be the region $\mathbb{C}-\left\{\alpha_{n j}^{-1} ; n\right.$ even $\}$ with the intention of applying Lemma 8.2. Take the lexicographical order on the set of all factors

$$
1-\alpha_{n j} t \text {, for } n \text { odd; } \frac{1}{1-\alpha_{n j} t}, \text { for } n \text { even. }
$$

Each factor is an analytic function on $U$. The sum $\sum_{n}\left(f_{n}(z)-1\right)$ here is equal to

$$
\sum_{n \text { odd }, j}\left(-\alpha_{n j} t\right)+\sum_{n \text { even }, j} \frac{\alpha_{n j} t}{1-\alpha_{n j} t} .
$$


Let

$$
g_{n}(t)=\left\{\begin{array}{cc}
\sum_{j=1}^{m_{n}}\left|\alpha_{n j} t\right|, & n \text { odd, } \\
\sum_{j=1}^{m_{n}} \frac{\left|\alpha_{n j} t\right|}{\left|1-\alpha_{n j} t\right|}, & n \text { even. }
\end{array}\right.
$$

We need to show that $\sum_{n} g_{n}(t)$ is pointwise convergent, uniformly on compact subsets of $U$. Precisely, we want to show that for any compact subset $B \subset U$, and for any $\varepsilon>0$, there exists a constant $N_{B} \in \mathbb{Z}$ such that

$$
\sum_{n \leq N} g_{n}(t)<\varepsilon
$$

for all $N \leq N_{B}$ and $t \in B$. Since $g_{n}(t)$ are nonnegative, it suffices to do this for $N=N_{B}$. There exists a constant $M_{B}$ such that $|t|<M_{B}$ for all $t \in B$. Since $\sum_{n, j}\left|\alpha_{n j}\right|$ converges, $\left|\alpha_{n j}\right| \rightarrow 0$ as $n \rightarrow-\infty$, and there exists a constant $L_{B} \in \mathbb{Z}$ such that $\left|\alpha_{n j}\right|<1 /\left(2 M_{B}\right)$ for all $n<L_{B}$. So

$$
g_{n}(t) \leq 2 \sum_{j=1}^{m_{n}}\left|\alpha_{n j} t\right|
$$

for all $n<L_{B}$ and $t \in B$. There exists a constant $N_{B}<L_{B}$ such that

$$
\sum_{n \leq N_{B}} \sum_{j}\left|\alpha_{n j}\right|<\varepsilon /\left(2 M_{B}\right)
$$

and hence

$$
\sum_{n \leq N_{B}} g_{n}(t) \leq 2 \sum_{n \leq N_{B}} \sum_{j}\left|\alpha_{n j} t\right| \leq 2 M_{B} \sum_{n \leq N_{B}} \sum_{j}\left|\alpha_{n j}\right|<\varepsilon .
$$

By Lemma 8.2, $L\left(\mathscr{Q}_{0}, K_{0}, t\right)$ extends to an analytic function on $U$. By the second part of Lemma 8.2, the $\alpha_{n j}^{-1}$, s, for $n$ even, are at worst poles rather than essential singularities, therefore the $L$-series is meromorphic on $\mathbb{C}$.

Now $L\left(\mathscr{C}_{0}, K_{0}, t\right)$ can be called an " $L$-function".

\section{Weight theorem for algebraic stacks}

9.1. We prove Theorem 1.4 in this section. For the reader's convenience, we briefly review the definition of the dimension of a locally noetherian $S$-algebraic stack $\mathscr{X}$ from [Laumon and Moret-Bailly 2000, Chapter 11].

If $X$ is a locally noetherian $S$-algebraic space and $x$ is a point of $X$, the dimension $\operatorname{dim}_{x} X$ of $X$ at $x$ is defined to be $\operatorname{dim}_{x^{\prime}} X^{\prime}$, for any pair $\left(X^{\prime}, x^{\prime}\right)$ where $X^{\prime}$ is an $S$-scheme étale over $X$ and $x^{\prime} \in X^{\prime}$ maps to $x$. This is independent of the choice of 
the pair. If $f: X \rightarrow Y$ is a morphism of $S$-algebraic spaces, locally of finite type, and $x$ is a point of $X$ with image $y$ in $Y$, then the relative $\operatorname{dimension} \operatorname{dim}_{x} f$ of $f$ at $x$ is defined to be $\operatorname{dim}_{x} X_{y}$.

Let $P: X \rightarrow \mathscr{X}$ be a presentation of an $S$-algebraic stack $\mathscr{X}$, and let $x$ be a point of $X$. Then the relative $\operatorname{dimension}_{x} \operatorname{dim}_{x} P$ of $P$ at $x$ is defined to be the relative dimension at $(x, x)$ of the smooth morphism $\mathrm{pr}_{1}: X \times \mathscr{x} X \rightarrow X$ of $S$-algebraic spaces.

If $\mathscr{X}$ is a locally noetherian $S$-algebraic stack and if $\xi$ is a point of $\mathscr{X}$, the dimension of $\mathscr{X}$ at $\xi$ is defined to be $\operatorname{dim}_{\xi} \mathscr{X}=\operatorname{dim}_{x} X-\operatorname{dim}_{x} P$, where $P: X \rightarrow \mathscr{X}$ is an arbitrary presentation of $\mathscr{L}$ and $x$ is an arbitrary point of $X$ lying over $\xi$. This definition is independent of all the choices made. At last one defines the dimension of $\mathscr{X}$ by $\operatorname{dim} \mathscr{X}=\sup _{\xi} \operatorname{dim}_{\xi} \mathscr{X}$. For quotient stacks we have $\operatorname{dim}[X / G]=\operatorname{dim} X-\operatorname{dim} G$.

Now we prove Theorem 1.4.

Proof. If $j: \mathscr{U}_{0} \rightarrow \mathscr{X}_{0}$ is an open substack with complement $i: \mathscr{L}_{0} \rightarrow \mathscr{X}_{0}$, then we have an exact sequence

$$
\cdots \longrightarrow H_{c}^{n}\left(U, j^{*} \mathscr{F}\right) \longrightarrow H_{c}^{n}(\mathscr{X}, \mathscr{F}) \longrightarrow H_{c}^{n}\left(\mathscr{E}, i^{*} \mathscr{F}\right) \longrightarrow \cdots .
$$

If both $H_{c}^{n}\left(\mathcal{U}, j^{*} \mathscr{F}\right)$ and $H_{c}^{n}\left(\mathscr{E}, i^{*} \mathscr{F}\right)$ are zero (respectively have all $\iota$-weights $\leq m$ for some number $m$ ), then so is $H_{c}^{n}(\mathscr{X}, \mathscr{F})$. Since the dimensions of $\mathscr{U}_{0}$ and $\mathscr{L}_{0}$ are no more than that of $\mathscr{X}_{0}$, and the set of punctual $\iota$-weights of $i^{*} \mathscr{F}_{0}$ and of $j^{*} \mathscr{F}_{0}$ is the same as that of $\mathscr{F}_{0}$, we may shrink $\mathscr{X}_{0}$ to a nonempty open substack. We can also make any finite base change on $\mathbb{F}_{q}$. To simplify notation, we may use twist (see 2.4) and projection formula to assume $w=0$. As before, we reduce to the case when $\mathscr{X}_{0}$ is geometrically connected, and the inertia $f: \mathscr{I}_{0} \rightarrow \mathscr{X}_{0}$ is flat, with rigidification $\pi: \mathscr{X}_{0} \rightarrow X_{0}$, where $X_{0}$ is a scheme. The squares in the following diagram are 2-Cartesian:

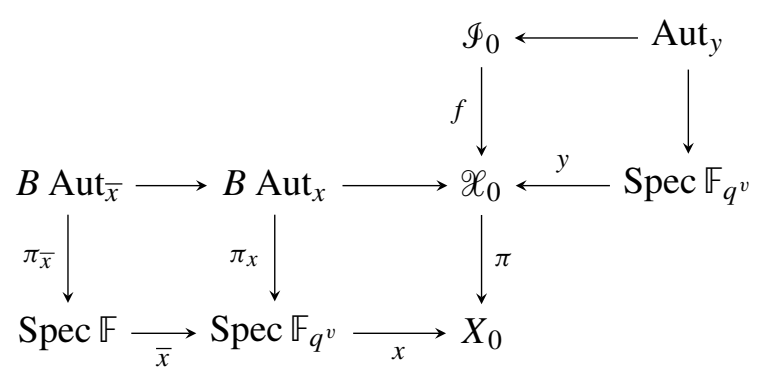

We have $\left(R^{k} \pi ! \mathscr{F}_{0}\right)_{\bar{x}}=H_{c}^{k}\left(B\right.$ Aut $\left._{\bar{x}}, \mathscr{F}\right)$. Since $f$ is representable and flat, and $\mathscr{X}_{0}$ is connected, all automorphism groups $\mathrm{Aut}_{x}$ have the same dimension, say $d$.

Assume Theorem 1.4 holds for all $B G_{0}$, where $G_{0}$ are $\mathbb{F}_{q}$-algebraic groups. Then $R^{k} \pi_{!} \mathscr{F}_{0}=0$ for $k>-2 d$, and for $k \leq-2 d$, the punctual $\iota$-weights of 
$R^{k} \pi_{!} \mathscr{F}_{0}$ are $\leq \frac{k}{2}-d$, hence by [Deligne 1980, 3.3.4], the punctual $\iota$-weights of $H_{c}^{r}\left(X, R^{k} \pi ! \mathscr{F}\right)$ are $\leq \frac{k}{2}-d+r$. Consider the Leray spectral sequence

$$
E_{2}^{r k}=H_{c}^{r}\left(X, R^{k} \pi ! \mathscr{F}\right) \Longrightarrow H_{c}^{r+k}(\mathscr{X}, \mathscr{F}) .
$$

If we maximize $\frac{k}{2}-d+r$ under the constraints

$$
r+k=n, \quad 0 \leq r \leq 2 \operatorname{dim} X_{0} \quad \text { and } \quad k \leq-2 d,
$$

we find that $H_{c}^{n}(\mathscr{X}, \mathscr{F})=0$ for $n>2 \operatorname{dim} X_{0}-2 d=2 \operatorname{dim} \mathscr{X}_{0}$, and for $n \leq 2 \operatorname{dim} \mathscr{X}_{0}$, the punctual $\iota$-weights of $H_{c}^{n}(\mathscr{X}, \mathscr{F})$ are $\leq \operatorname{dim} X_{0}+\frac{n}{2}-d=\operatorname{dim} \mathscr{X}_{0}+\frac{n}{2}$.

So we reduce to the case $\mathscr{X}_{0}=B G_{0}$. By Lemma 4.6 the Leray spectral sequence for $h: B G_{0} \rightarrow B \pi_{0}\left(G_{0}\right)$ degenerates to isomorphisms

$$
H_{c}^{0}\left(B \pi_{0}(G), R^{n} h_{!} \mathscr{F}\right) \simeq H_{c}^{n}(B G, \mathscr{F}) .
$$

The fibers of $h$ are isomorphic to $B G_{0}^{0}$, so by base change and Lemma 4.6 we reduce to the case when $G_{0}$ is connected. Let $d=\operatorname{dim} G_{0}$ and $f: B G_{0} \rightarrow \operatorname{Spec} \mathbb{F}_{q}$ be the structural map. In this case, $\mathscr{F}_{0} \cong f^{*} V$ for some $\overline{\mathbb{Q}}_{\ell}$-representation $V$ of $W\left(\mathbb{F}_{q}\right)$, and hence $\mathscr{F}_{0}$ and $V$ have the same punctual $\iota$-weights. Using the natural isomorphism $H_{c}^{n}(B G) \otimes V \simeq H_{c}^{n}(B G, \mathscr{F})$, we reduce to the case when $\mathscr{F}_{0}=\overline{\mathbb{Q}}_{\ell}$. In 4.8.2 we see that, if $\alpha_{i 1}, \ldots, \alpha_{i n_{i}}$ are the eigenvalues of $F$ on $N^{i}, i \geq 1$ odd, then the eigenvalues of $F$ on $H_{c}^{-2 k-2 d}(B G)$ are

$$
q^{-d} \prod_{i, j} \alpha_{i j}^{-m_{i j}}, \quad \text { where } \sum_{i, j} m_{i j}(i+1)=2 k .
$$

Since $i \geq 1$, we have $\sum i m_{i j} \geq k$; together with $\left|\alpha_{i j}\right| \geq q^{i / 2}$ one deduces

$$
\left|q^{-d} \prod_{i, j} \alpha_{i j}^{-m_{i j}}\right| \leq q^{(-k-2 d) / 2},
$$

so the punctual $\iota$-weights of $H_{c}^{-2 k-2 d}(B G)$ are $\leq-k-2 d$ for $k \geq 0$, and the other compactly supported cohomology groups are zero.

It is clear from the proof and [Deligne 1980,3.3.10] that the weights of $H_{c}^{n}(\mathscr{X}, \mathscr{F})$ differ from the weights of $\mathscr{F}_{0}$ by integers.

Recall that $H^{2 k}(B G)$ is pure of weight $2 k$, for a linear algebraic group $G_{0}$ over $\mathbb{F}_{q}$ [Deligne 1974b, 9.1.4]. Therefore, $H_{c}^{-2 k-2 d}(B G)$ is pure of weight $-2 k-2 d$, and following the same proof as above, we are done.

Remark 9.2. When $\mathscr{X}_{0}=X_{0}$ is a scheme, and $n \leq 2 \operatorname{dim} X_{0}$, we have

$$
\operatorname{dim} X_{0}+\frac{n}{2}+w \geq n+w,
$$


so our bound for weights is worse than the bound in [Deligne 1980, 3.3.4]. For an $\mathbb{F}_{q}$-abelian variety $A$, our bound for the weights of $H_{c}^{n}(B A)$ is sharp: the weights are exactly $\operatorname{dim}(B A)+\frac{n}{2}$, whenever the cohomology group is nonzero.

We hope Theorem 1.4 has useful and interesting applications, for instance for generalizing the decomposition theorem of Beilinson-Bernstein-Deligne-Gabber (see [Sun 2012]) to stacks with affine stabilizers, and for studying the Hasse-Weil zeta functions of Artin stacks over number fields. For instance, it implies that the Hasse-Weil zeta function is analytic in some right half complex $s$-plane.

Using Theorem 1.4 we can show certain stacks have $\mathbb{F}_{q}$-points.

Example 9.3. Let $\mathscr{X}_{0}$ be a form of $B \mathbb{G}_{m}$, that is, $\mathscr{X} \cong B \mathbb{G}_{m, \mathbb{F}}$ over $\mathbb{F}$. Then all the automorphism group schemes in $\mathscr{X}_{0}$ are affine, and for any $n \geq 0$ we have $h_{c}^{-2-2 n}(\mathscr{C})=h_{c}^{-2-2 n}\left(B \mathbb{G}_{m}\right)=1$. Let $\alpha_{-2-2 n}$ be the eigenvalue of $F$ on $H_{c}^{-2-2 n}(\mathscr{C})$. Then by Theorem 1.4 we have $\left|\alpha_{-2-2 n}\right| \leq q^{-1-n}$. Smoothness is fppf local on the base, so $\mathscr{X}_{0}$ is smooth and connected, hence $H_{c}^{-2}(\mathscr{X})=\overline{\mathbb{Q}}_{\ell}(1)$ and $\alpha_{-2}=q^{-1}$. So

$$
\begin{aligned}
\# \mathscr{Q}_{0}\left(\mathbb{F}_{q}\right)=\sum_{n \geq 0} \operatorname{Tr}\left(F, H_{c}^{-2-2 n}(\mathscr{X})\right) & =q^{-1}+\alpha_{-4}+\alpha_{-6}+\cdots \\
& \geq q^{-1}-q^{-2}-q^{-3}+\cdots=q^{-1}-\frac{q^{-1}}{q-1}>0
\end{aligned}
$$

when $q \neq 2$. In fact, since there exists an integer $r \geq 1$ such that $\mathscr{X}_{0} \otimes \mathbb{F}_{q^{r}} \cong B \mathbb{G}_{m, \mathbb{F}_{q} r}$, we see that all cohomology groups $H_{c}^{-2-2 n}(\mathscr{X})$ are pure, that is, $\left|\alpha_{-2-2 n}\right|=q^{-1-n}$.

In fact, one can classify the forms of $B \mathbb{G}_{m, \mathbb{F}_{q}}$ as follows. If $\mathscr{L}_{0}$ is a form, then it is also a gerbe over $\operatorname{Spec} \mathbb{F}_{q}$, hence a neutral gerbe $B G_{0}$ for some algebraic group $G_{0}$ by Behrend [2003, 6.4.2]. By comparing the automorphism groups, we see that $G_{0}$ is a form of $\mathbb{G}_{m, \mathbb{F}_{q}}$. There is only one nontrivial form of $\mathbb{G}_{m, \mathbb{F}_{q}}$, because

$$
H^{1}\left(\mathbb{F}_{q}, \operatorname{Aut}\left(\mathbb{G}_{m}\right)\right)=H^{1}\left(\mathbb{F}_{q}, \mathbb{Z} / 2 \mathbb{Z}\right)=\mathbb{Z} / 2 \mathbb{Z},
$$

and this form is the kernel $R_{\mathbb{F}_{q^{2}} / \mathbb{F}_{q}}^{1} \mathbb{G}_{m, \mathbb{F}_{q^{2}}}$ of the norm map

$$
R_{\mathbb{F}_{q^{2}} / \mathbb{F}_{q}} \mathbb{G}_{m, \mathbb{F}_{q^{2}}} \stackrel{\mathrm{Nm}}{\longrightarrow} \mathbb{G}_{m, \mathbb{F}_{q}},
$$

where $R_{\mathbb{F}_{q^{2}} / \mathbb{F}_{q}}$ is the operation of Weil's restriction of scalars. Therefore, the only nontrivial form of $B \mathbb{G}_{m, \mathbb{F}_{q}}$ is $B\left(R_{\mathbb{F}_{q^{2}} / \mathbb{F}_{q}}^{1} \mathbb{G}_{m, \mathbb{F}_{q^{2}}}\right)$. In particular, they all have $\mathbb{F}_{q^{-}}$ points, even when $q=2$.

Example 9.4. Consider the projective line $\mathbb{P}^{1}$ with the following action of $\mathbb{G}_{m}$ : it acts by multiplication on the open part $\mathbb{A}^{1} \subset \mathbb{P}^{1}$, and leaves the point $\infty$ fixed. So we get a quotient stack $\left[\mathbb{P}^{1} / \mathbb{G}_{m}\right]$ over $\mathbb{F}_{q}$. Let $\mathscr{X}_{0}$ be a form of $\left[\mathbb{P}^{1} / \mathbb{G}_{m}\right]$. We want to find an $\mathbb{F}_{q}$-point on $\mathscr{X}_{0}$, or even better, an $\mathbb{F}_{q}$-point on $\mathscr{X}_{0}$ which, when considered as a point in $\mathscr{X}(\mathbb{F}) \cong\left[\mathbb{P}^{1} / \mathbb{G}_{m}\right](\mathbb{F})$, lies in the open dense orbit $\left[\mathbb{G}_{m} / \mathbb{G}_{m}\right](\mathbb{F})$. 
9.4.1. Consider the following general situation. Let $G_{0}$ be a connected $\mathbb{F}_{q}$-algebraic group, and let $X_{0}$ be a proper smooth variety with a $G_{0}$-action over $\mathbb{F}_{q}$. Let

$$
\left[X_{0} / G_{0}\right] \stackrel{f}{\longrightarrow} B G_{0} \stackrel{g}{\longrightarrow} \operatorname{Spec} \mathbb{F}_{q}
$$

be the natural maps, and let $\mathscr{L}_{0}$ be a form of $\left[X_{0} / G_{0}\right]$. Then $f$ is representable and proper. For every $k, R^{k} f_{*} \overline{\mathbb{Q}}_{\ell}$ is a lisse sheaf, and takes the form $g^{*} V_{k}$ for some sheaf $V_{k}$ on Spec $\mathbb{F}_{q}$. Consider the Leray spectral sequence

$$
E_{2}^{r k}=R^{r} g_{!} R^{k} f_{*} \overline{\mathbb{Q}}_{\ell} \Longrightarrow R^{r+k}(g f) ! \overline{\mathbb{Q}}_{\ell} .
$$

Since $R^{r} g_{!} R^{k} f_{*} \overline{\mathbb{Q}}_{\ell}=R^{r} g_{!}\left(g^{*} V_{k}\right)=\left(R^{r} g_{!} \overline{\mathbb{Q}}_{\ell}\right) \otimes V_{k}$, we have

$$
h_{c}^{n}(\mathscr{X})=h_{c}^{n}([X / G]) \leq \sum_{r+k=n} h_{c}^{r}(B G) \cdot \operatorname{dim} V_{k}=\sum_{r+k=n} h_{c}^{r}(B G) \cdot h^{k}(X) .
$$

Now we return to $\left[\mathbb{P}^{1} / \mathbb{G}_{m}\right]$. Since $h^{0}\left(\mathbb{P}^{1}\right)=h^{2}\left(\mathbb{P}^{1}\right)=1$ and $h_{c}^{-2 i}\left(B \mathbb{G}_{m}\right)=1$ for $i \geq 1$, we see that $h_{c}^{n}(\mathscr{X})=0$ for $n$ odd and

$$
h_{c}^{2 n}(\mathscr{X}) \leq h^{0}\left(\mathbb{P}^{1}\right) h_{c}^{2 n}\left(B \mathbb{G}_{m}\right)+h^{2}\left(\mathbb{P}^{1}\right) h_{c}^{2 n-2}\left(B \mathbb{G}_{m}\right)= \begin{cases}0, & n \geq 1, \\ 1, & n=0, \\ 2, & n<0 .\end{cases}
$$

Since $\mathscr{X}_{0}$ is connected and smooth of dimension 0 , we have $H_{c}^{0}(\mathscr{X})=\overline{\mathbb{Q}}_{\ell}$. By Theorem 1.4, the $\iota$-weights of $H_{c}^{2 n}(\mathscr{X})$ are $\leq 2 n$. The trace formula gives

$$
\begin{aligned}
\# \mathscr{L}_{0}\left(\mathbb{F}_{q}\right)=\sum_{n \leq 0} \operatorname{Tr}\left(F, H_{c}^{2 n}(\mathscr{X})\right)=1+\sum_{n<0} \operatorname{Tr}\left(F, H_{c}^{2 n}(\mathscr{X})\right) \\
\quad \geq 1-2 \sum_{n<0} q^{n}=1-\frac{2}{q-1}>0
\end{aligned}
$$

when $q \geq 4$.

In order for the rational point to be in the open dense orbit, we need an upper bound for the number of $\mathbb{F}_{q}$-points on the closed orbits. When passing to $\mathbb{E}$, there are 2 closed orbits, both having stabilizer $\mathbb{G}_{m, \mathbb{F}}$. So in $\left[\mathscr{L}_{0}\left(\mathbb{F}_{q}\right)\right]$ there are at most 2 points whose automorphism groups are forms of the algebraic group $\mathbb{G}_{m}, \mathbb{F}_{q}$. From the cohomology sequence

$$
1 \longrightarrow\left(R_{\mathbb{F}_{q^{2}} / \mathbb{F}_{q}}^{1} \mathbb{G}_{m, \mathbb{F}_{q^{2}}}\right)\left(\mathbb{F}_{q}\right) \longrightarrow \mathbb{F}_{q^{2}}^{*} \stackrel{\mathrm{Nm}}{\longrightarrow} \mathbb{F}_{q}^{*}
$$

we see that

$$
\#\left(R_{\mathbb{F}_{q^{2}} / \mathbb{F}_{q}}^{1} \mathbb{G}_{m, \mathbb{F}_{q^{2}}}\right)\left(\mathbb{F}_{q}\right)=q+1 .
$$

Since $1 /(q+1) \leq 1 /(q-1)$, the space that the closed orbits can take is at most $2 /(q-1)$, and equality holds only when the two closed orbits are both defined 
over $\mathbb{F}_{q}$ with stabilizer $\mathbb{G}_{m}$. In order for there to exist an $\mathbb{F}_{q}$-point in the open dense orbit, we need

$$
1-\frac{2}{q-1}>\frac{2}{q-1}
$$

and this is so when $q \geq 7$.

\section{About independence of $\ell$}

The coefficients of the expansion of the infinite product

$$
Z\left(\mathscr{X}_{0}, t\right)=\prod_{i \in \mathbb{Z}} P_{i, \ell}\left(\mathscr{X}_{0}, t\right)^{(-1)^{i+1}}
$$

are rational numbers and are independent of $\ell$, because the $c_{v}\left(\mathscr{Q}_{0}\right)$ 's are rational numbers independent of $\ell$. A famous conjecture is that this is also true for each $P_{i, \ell}\left(\mathscr{C}_{0}, t\right)$. First we show that the roots of $P_{i, \ell}\left(\mathscr{X}_{0}, t\right)$ are Weil $q$-numbers. Note that $P_{i, \ell}\left(\mathscr{Q}_{0}, t\right) \in \mathbb{Q}_{\ell}[t]$.

Definition 10.1. An algebraic number is called a Weil $q$-number if all of its conjugates have the same weight relative to $q$, and this weight is a rational integer. It is called $a$ Weil $q$-integer if in addition it is an algebraic integer. A number in $\overline{\mathbb{Q}}_{\ell}$ is called a Weil $q$-number if it is a Weil q-number via $\iota$.

For $\alpha \in \overline{\mathbb{Q}}_{\ell}$, being a Weil $q$-number or not is independent of $\iota$; in fact the images in $\mathbb{C}$ under various $\iota$ 's are conjugate.

For an $\mathbb{F}_{q}$-variety $X_{0}$, not necessarily smooth or proper, [Deligne 1980, 3.3.4] implies all Frobenius eigenvalues of $H_{c}^{i}(X)$ are Weil $q$-integers. The following lemma generalizes this.

Lemma 10.2. For every $\mathbb{F}_{q}$-algebraic stack $\mathscr{X}_{0}$, and a prime number $\ell \neq p$, the roots of each $P_{i, \ell}\left(\mathscr{Q}_{0}, t\right)$ are Weil q-numbers. In particular, the coefficients of $P_{i, \ell}\left(\mathscr{Q}_{0}, t\right)$ are algebraic numbers in $\mathbb{Q}_{\ell}$ (that is, algebraic over $\left.\mathbb{Q}\right)$.

Proof. For an open immersion $j: \mathscr{U}_{0} \rightarrow \mathscr{X}_{0}$ with complement $i: \mathscr{L}_{0} \rightarrow \mathscr{X}_{0}$, we have an exact sequence

$$
\cdots \longrightarrow H_{c}^{i}(\mathscr{U}) \longrightarrow H_{c}^{i}(\mathscr{X}) \longrightarrow H_{c}^{i}(\mathscr{L}) \longrightarrow \cdots,
$$

thus we may shrink to a nonempty open substack. In particular, Lemma 10.2 holds for algebraic spaces, by Knutson [1971, II 6.7] and Deligne [1980, 3.3.4].

We may assume $\mathscr{X}_{0}$ is smooth and connected. By Poincaré duality, it suffices to show that the Frobenius eigenvalues of $H^{i}(\mathscr{X})$ are Weil $q$-numbers, for all $i$. Take a presentation $X_{0} \rightarrow \mathscr{L}_{0}$ and consider the associated strictly simplicial smooth covering $X_{0}^{\bullet} \rightarrow \mathscr{X}_{0}$ by algebraic spaces. Then there is a spectral sequence [Laszlo 
and Olsson 2008b, 10.0.9]

$$
E_{1}^{r k}=H^{k}\left(X^{r}\right) \Longrightarrow H^{r+k}(\mathscr{X}),
$$

and the assertion for $\mathscr{L}_{0}$ follows from the assertion for algebraic spaces.

Problem 10.3. Is each

$$
P_{i, \ell}\left(\mathscr{Q}_{0}, t\right)=\operatorname{det}\left(1-F t, H_{c}^{i}\left(\mathscr{X}, \mathbb{Q}_{\ell}\right)\right)
$$

a polynomial with coefficients in $\mathbb{Q}$, and the coefficients are independent of $\ell$ ?

Remark 10.3.1. (i) Note that, unlike the case for varieties, we cannot expect the coefficients to be integers (for instance, for $B \mathbb{G}_{m}$, the coefficients are $1 / q^{i}$ ).

(ii) Problem 10.3 is known to be true for smooth proper varieties [Deligne 1980, 3.3.9], and (coarse moduli spaces of) proper smooth algebraic stacks of finite diagonal (see Proposition 7.3.2). It remains open for general varieties. Even the Betti numbers are not known to be independent of $\ell$ for a general variety, see [Illusie 2006].

Let us give positive answer to Problem 10.3 in some special cases of algebraic stacks. In Section 7 we see that it holds for $B E$ and $B G L_{N}$. We can generalize these two cases as follows.

Lemma 10.4. Problem 10.3 has a positive answer for:

(i) $B A$, where $A$ is an $\mathbb{F}_{q}$-abelian variety.

(ii) $B G_{0}$, where $G_{0}$ is a linear algebraic group over $\mathbb{F}_{q}$.

Proof. (i) Let $g=\operatorname{dim} A$. Then $N=H^{1}(A)$ is a $2 g$-dimensional vector space, with eigenvalues $\alpha_{1}, \ldots, \alpha_{2 g}$ for the Frobenius action $F$, and $N$ is pure of weight 1 . Let $a_{1}, \ldots, a_{2 g}$ be a basis for $N$ so that $F$ is given by the upper-triangular matrix

$$
\left[\begin{array}{ccc}
\alpha_{1} & * & * \\
& \ddots & * \\
& & \alpha_{2 g}
\end{array}\right] .
$$

Then $H^{*}(B A)=\operatorname{Sym}^{*} N[-1]=\overline{\mathbb{Q}}_{\ell}\left[a_{1}, \ldots, a_{2 g}\right]$, where each $a_{i}$ sits in degree 2 . In degree $2 n, H^{2 n}(B A)=\overline{\mathbb{Q}}_{\ell}\left\langle a_{i_{1}} \cdots a_{i_{n}} \mid 1 \leq i_{1}, \ldots, i_{n} \leq 2 g\right\rangle$, and the eigenvalues are $\alpha_{i_{1}} \cdots \alpha_{i_{n}}$. By Poincaré duality

$$
H_{c}^{-2 n-2 g}(B A)=H^{2 n}(B A)^{\vee} \otimes \overline{\mathbb{Q}}_{\ell}(g)
$$

we see that the eigenvalues of $F$ on $H_{c}^{-2 g-2 n}(B A)$ are

$$
q^{-g} \cdot \alpha_{i_{1}}^{-1} \cdots \alpha_{i_{n}}^{-1}
$$


Each factor

$$
P_{-2 g-2 n}\left(q^{g} t\right)=\prod_{1<i_{1}}\left(1-\left(\alpha_{i_{1}} \cdots \alpha_{i_{n}}\right)^{-1} t\right)
$$

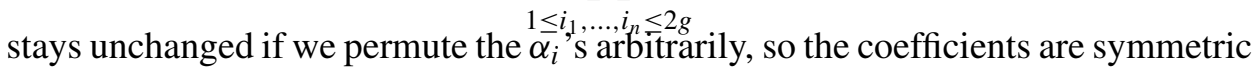
polynomials in the $\alpha_{i}^{-1}$,s with integer coefficients, hence are polynomials in the elementary symmetric functions, which are coefficients of $\prod_{i=1}^{2 g}\left(t-\alpha_{i}^{-1}\right)$. The polynomial

$$
\prod_{i=1}^{2 g}\left(1-\alpha_{i} t\right)=\operatorname{det}\left(1-F t, H^{1}\left(A, \mathbb{Q}_{\ell}\right)\right)
$$

also has roots $\alpha_{i}^{-1}$, and this is a polynomial with integer coefficients, independent of $\ell$, since $A$ is smooth and proper. Let $m= \pm q^{g}$ be leading coefficient of it. Then

$$
\prod_{i=1}^{2 g}\left(t-\alpha_{i}^{-1}\right)=\frac{1}{m} \prod_{i=1}^{2 g}\left(1-\alpha_{i} t\right) .
$$

This verifies Problem 10.3 for $B A$.

(ii) Let $d=\operatorname{dim} G_{0}$. For every $k \geq 0, H^{2 k}(B G)$ is pure of weight $2 k$ [Deligne $1974 \mathrm{~b}, 9.1 .4]$, hence $H_{c}^{-2 d-2 k}(B G)$ is pure of weight $-2 d-2 k$ by Poincare duality. The entire function

$$
\frac{1}{Z\left(B G_{0}, t\right)}=\prod_{k \geq 0} P_{-2 d-2 k}\left(B G_{0}, t\right) \in \mathbb{Q}[[t]]
$$

is independent of $\ell$, and invariant under the action of $\mathrm{Gal}(\mathbb{Q})$ on the coefficients of the Taylor expansion. Therefore the roots of $P_{-2 d-2 k}\left(B G_{0}, t\right)$ can be described as

$$
\text { "zeros of } \frac{1}{Z\left(B G_{0}, t\right)} \text { that have weight } 2 d+2 k \text { relative to } q, "
$$

which is a description independent of $\ell$, and these roots (which are algebraic numbers) are permuted under $\operatorname{Gal}(\mathbb{Q})$. Hence $P_{-2 d-2 k}\left(B G_{0}, t\right)$ has rational coefficients.

The following proposition generalizes Proposition 7.3.2 and Lemma 10.4 (ii).

Proposition 10.5. Let $X_{0}$ be the coarse moduli space of a proper smooth $\mathbb{F}_{q^{-}}$ algebraic stack of finite diagonal, and let $G_{0}$ be a linear $\mathbb{F}_{q}$-algebraic group that acts on $X_{0}$, and let $\mathscr{X}_{0}$ be a form of the quotient stack $\left[X_{0} / G_{0}\right]$. Then Problem 10.3 is verified for $\mathscr{L}_{0}$.

Proof. It suffices to show that $H_{c}^{n}(\mathscr{L})$ is pure of weight $n$, for every $n$. To show this, we can make a finite extension of the base field $\mathbb{F}_{q}$, so we may assume that $\mathscr{L}_{0}=\left[X_{0} / G_{0}\right]$. Let

$$
\mathscr{X}_{0} \stackrel{f}{\longrightarrow} B G_{0} \stackrel{h}{\longrightarrow} B \pi_{0}\left(G_{0}\right)
$$


be the natural maps.

Let $d=\operatorname{dim} G_{0}$. Consider the spectral sequence

$$
H_{c}^{-2 d-2 r}\left(B G, R^{k} f_{!} \overline{\mathbb{Q}}_{\ell}\right) \Longrightarrow H_{c}^{-2 d-2 r+k}(\mathscr{X}) .
$$

The $E_{2}$-terms can be computed from the degenerate Leray spectral sequence for $h$ :

$$
H_{c}^{-2 d-2 r}\left(B G, R^{k} f_{!} \overline{\mathbb{Q}}_{\ell}\right) \simeq H_{c}^{0}\left(B \pi_{0}(G), R^{-2 d-2 r} h_{!} R^{k} f_{!} \overline{\mathbb{Q}}_{\ell}\right) .
$$

We remark that the restriction of $R^{-2 d-2 r} h_{!} R^{k} f_{!} \overline{\mathbb{Q}}_{\ell}$ along the natural projection Spec $\mathbb{F}_{q} \rightarrow B \pi_{0}\left(G_{0}\right)$ is isomorphic to the Galois module $H_{c}^{-2 d-2 r}\left(B G^{0}, R^{k} f_{!} \overline{\mathbb{Q}}_{\ell}\right)$, and since $G_{0}^{0}$ is connected, $\left.\left(R^{k} f_{!} \overline{\mathbb{Q}}_{\ell}\right)\right|_{B G_{0}^{0}}$ is the inverse image of some sheaf $V_{k}$ via the structural map $B G_{0}^{0} \rightarrow \operatorname{Spec} \mathbb{F}_{q}$. By base change, we see that the sheaf $V_{k}$, regarded as a $\mathrm{Gal}\left(\mathbb{F}_{q}\right)$-module, is $H^{k}(X)$. By projection formula we have

$$
H_{c}^{-2 d-2 r}\left(B G^{0}, R^{k} f_{!} \overline{\mathbb{Q}}_{\ell}\right) \simeq H_{c}^{-2 d-2 r}\left(B G^{0}\right) \otimes H^{k}(X)
$$

as representations of $\mathrm{Gal}\left(\mathbb{F}_{q}\right)$, and by Proposition 7.3.2, the right hand side is pure of weight $-2 d-2 r+k$. By Lemma 4.6, $H_{c}^{-2 d-2 r}\left(B G, R^{k} f_{!} \overline{\mathbb{Q}}_{\ell}\right)$ is also pure of weight $-2 d-2 r+k$, therefore $H_{c}^{n}(\mathscr{L})$ is pure of weight $n$, for every $n$.

10.6. Finally, let us consider the following much weaker version of independence of $\ell$. For $\mathscr{X}_{0}$ and $i \in \mathbb{Z}$, let $\Psi\left(\mathscr{X}_{0}, i\right)$ be the following property: the Frobenius eigenvalues of $H_{c}^{i}\left(\mathscr{Q}, \overline{\mathbb{Q}}_{\ell}\right)$, counted with multiplicity, for all $\ell \neq p$, are contained in a finite set of algebraic numbers with multiplicities assigned, and this set together with the assignment of multiplicity, depends only on $\mathscr{X}_{0}$ and $i$. In particular it is independent of $\ell$. In other words, there is a finite decomposition of the set of all prime numbers $\ell \neq p$ into disjoint union of some subsets, such that the Frobenius eigenvalues of $H_{c}^{i}\left(\mathscr{X}, \overline{\mathbb{Q}}_{\ell}\right)$ depends only on the subset that $\ell$ belongs to. If this property holds, we also denote such a finite set of algebraic numbers (which is not unique) by $\Psi\left(\mathscr{L}_{0}, i\right)$, if there is no confusion.

Proposition 10.6.1. The property $\Psi\left(\mathscr{X}_{0}, i\right)$ holds for every $\mathscr{X}_{0}$ and $i$.

Proof. If $\bigcup_{0}$ is an open substack of $\mathscr{X}_{0}$ with complement $\mathscr{L}_{0}$, and properties $\Psi\left(\mathscr{U}_{0}, i\right)$ and $\Psi\left(\mathscr{L}_{0}, i\right)$ hold, then $\Psi\left(\mathscr{X}_{0}, i\right)$ also holds, and the finite set $\Psi\left(\mathscr{X}_{0}, i\right)$ a subset of $\Psi\left(\mathscr{U}_{0}, i\right) \cup \Psi\left(\mathscr{L}_{0}, i\right)$.

Firstly we prove this for schemes $X_{0}$. By shrinking $X_{0}$ we can assume it is a connected smooth variety. By Poincaré duality it suffices to prove the similar statement $\Psi^{*}\left(X_{0}, i\right)$ for ordinary cohomology, that is, with $H_{c}^{i}$ replaced by $H^{i}$, for all $i$. This follows from [de Jong 1996] and [Deligne 1980, 3.3.9]. Therefore it also holds for all algebraic spaces.

For a general algebraic stack $\mathscr{X}_{0}$, by shrinking it we can assume it is connected smooth. By Poincaré duality, it suffices to prove $\Psi^{*}\left(\mathscr{C}_{0}, i\right)$ for all $i$. This can be 
done by taking a hypercover by simplicial algebraic spaces, and considering the associated spectral sequence.

\section{Acknowledgment}

I would like to thank my advisor Martin Olsson for introducing this topic to me, and giving so many suggestions during the writing. Weizhe Zheng sent me many helpful comments, and Yves Laszlo helped me to correct some inaccuracies in the first version of the paper. Many people, especially Brian Conrad and Matthew Emerton, have helped me on MathOverflow during the writing. The revision of the paper was done during the stay in Ecole polytechnique CMLS and Université Paris-Sud, while I was supported by ANR grant G-FIB.

\section{References}

[Behrend 1993] K. A. Behrend, "The Lefschetz trace formula for algebraic stacks", Invent. Math. 112:1 (1993), 127-149. MR 94d:14023 Zbl 0792.14005

[Behrend 2003] K. A. Behrend, "Derived $l$-adic categories for algebraic stacks", Mem. Amer. Math. Soc. 163:774 (2003), viii+93. MR 2004e:14006 Zbl 1051.14023

[Běllinson et al. 1982] A. A. Beřlinson, J. Bernstein, and P. Deligne, "Faisceaux pervers", pp. 5-171 in Analysis and topology on singular spaces, I (Luminy, 1981), Astérisque 100, Soc. Math. France, Paris, 1982. MR 86g:32015 Zbl 0536.14011

[Conrad 2005] B. Conrad, "Keel-Mori theorem via stacks", preprint, 2005, available at http://math. stanford.edu/ conrad/papers/coarsespace.pdf.

[Conway 1973] J. B. Conway, Functions of one complex variable, Graduate Texts in Mathematics 11, Springer, New York, 1973. MR 56 \#5843 Zbl 0277.30001

[Deligne 1974a] P. Deligne, "La conjecture de Weil. I", Inst. Hautes Études Sci. Publ. Math. 43 (1974), 273-307. MR 49 \#5013 Zbl 0287.14001

[Deligne 1974b] P. Deligne, “Théorie de Hodge. III”, Inst. Hautes Études Sci. Publ. Math. 44 (1974), 5-77. MR 58 \#16653b Zbl 0237.14003

[Deligne 1977] P. Deligne, Cohomologie étale (SGA 4 $\frac{1}{2}$ ), Lecture Notes in Math. 569, Springer, Berlin, 1977. MR 57 \#3132 Zbl 0345.00010

[Deligne 1980] P. Deligne, "La conjecture de Weil. II", Inst. Hautes Études Sci. Publ. Math. 52 (1980), 137-252. MR 83c:14017 Zbl 0456.14014

[Grothendieck 1960] A. Grothendieck, "Technique de descente et théorèmes d'existence en géométrie algébriques, II: Le théorème d'existence en théorie formelle des modules", in Séminaire Bourbaki 1961/1962 (Exposé 195), W. A. Benjamin, Amsterdam, 1960. Reprinted as pp. 369-390 in Séminaire Bourbaki 5, Soc. Math. France, Paris, 1995. MR 1603480 Zbl 0234.14007

[Grothendieck 1965] A. Grothendieck, "Formule de Lefschetz et rationalité des fonctions $L$ ", in Séminaire Bourbaki 1964/1965 (Exposé 279), W. A. Benjamin, Amsterdam, 1965. Reprinted as pp. 41-55 in Séminaire Bourbaki 9, Soc. Math. France, Paris, 1995. MR 1608788 Zbl 0199.24802

[Grothendieck and Demazure 1970] A. Grothendieck and M. Demazure (editors), Schémas en groupes, I: Propriétés générales des schémas en groupes (SGA 3), Lecture Notes in Mathematics 151, Springer, Berlin, 1970. MR 0274458 Zbl 0207.51401 
[Grothendieck and Raynaud 1971] A. Grothendieck and M. Raynaud, Revêtements étales et groupe fondamental (SGA 1), Lecture Notes in Mathematics 224, Springer, Berlin, 1971. MR 50 \#7129 Zbl 0234.14002

[Illusie 1977] L. Illusie (editor), Cohomologie $\ell$-adique et Fonctions L (SGA 5), Lecture Notes in Mathematics 589, Springer, Berlin, 1977. MR 0491704 (58 \#10907)

[Illusie 2006] L. Illusie, "Miscellany on traces in $\ell$-adic cohomology: a survey", Jpn. J. Math. 1:1 (2006), 107-136. MR 2007g:14016 Zbl 1156.14309

[Illusie et al. 2008] L. Illusie, Y. Laszlo, and F. Orgogozo, "Théorème de finitude en cohomologie étale, d'après Ofer Gabber", Lecture notes, 2008, available at http://www.math.polytechnique.fr/ laszlo/gdtgabber/gdtgabber.html.

[de Jong 1996] A. J. de Jong, "Smoothness, semi-stability and alterations", Inst. Hautes Études Sci. Publ. Math. 83 (1996), 51-93. MR 98e:14011 Zbl 0916.14005

[Kiehl and Weissauer 2001] R. Kiehl and R. Weissauer, Weil conjectures, perverse sheaves and l'adic Fourier transform, Ergebnisse der Math. (3) 42, Springer, Berlin, 2001. MR 2002k:14026 Zbl 0988.14009

[Knutson 1971] D. Knutson, Algebraic spaces, Lecture Notes in Mathematics 203, Springer, Berlin, 1971. MR 46 \#1791 Zbl 0221.14001

[Lang 1956] S. Lang, "Algebraic groups over finite fields", Amer. J. Math. 78 (1956), 555-563. MR 19,174a Zbl 0073.37901

[Laszlo and Olsson 2008a] Y. Laszlo and M. Olsson, "The six operations for sheaves on Artin stacks. I. Finite coefficients”, Publ. Math. Inst. Hautes Études Sci. 107 (2008), 109-168. MR 2009f:14003a Zbl 1191.14002

[Laszlo and Olsson 2008b] Y. Laszlo and M. Olsson, "The six operations for sheaves on Artin stacks. II. Adic coefficients”, Publ. Math. Inst. Hautes Études Sci. 107 (2008), 169-210. MR 2009f:14003b Zbl 1191.14003

[Laumon 2002] G. Laumon, "La correspondance de Langlands sur les corps de fonctions (d'après Laurent Lafforgue)”, pp. 207-265 in Séminaire Bourbaki 1999/2000 (Exposé 873), Astérisque 276, Société Mathématique de France, Paris, 2002. MR 2003b:11052 Zbl 1016.11052

[Laumon and Moret-Bailly 2000] G. Laumon and L. Moret-Bailly, Champs algébriques, Ergebnisse der Math. (3) 39, Springer, Berlin, 2000. MR 2001f:14006 Zbl 0945.14005

[Milne 2008] J. S. Milne, "Lectures on etale cohomology", 2008, available at http://www.jmilne.org/ math/CourseNotes/lec.html. Version 2.10.

[Milne 2012] J. S. Milne, "Basic theory of affine group schemes", 2012, available at http://www. jmilne.org/math/CourseNotes/ala.html. Version 1.00.

[Olsson 2007] M. Olsson, "Sheaves on Artin stacks", J. Reine Angew. Math. 603 (2007), 55-112. MR 2008b:14002 Zbl 1137.14004

[Olsson 2008a] M. Olsson, "Fujiwara's theorem for equivariant correspondences", 2008, available at http://math.berkeley.edu/ molsson/.

[Olsson 2008b] M. C. Olsson, Compactifying moduli spaces for abelian varieties, Lecture Notes in Mathematics 1958, Springer, Berlin, 2008. MR 2009h:14072 Zbl 1165.14004

[Riou 2007] J. Riou, "Dualité (d'après Ofer Gabber)", preprint, 2007, available at http://tinyurl.com/ cstafmq.

[Spaltenstein 1988] N. Spaltenstein, "Resolutions of unbounded complexes", Compositio Math. 65:2 (1988), 121-154. MR 89m:18013 Zbl 0636.18006 
[Sun 2012] S. Sun, "Decomposition theorem for perverse sheaves on Artin stacks over finite fields", preprint, 2012. To appear in Duke Math. J. arXiv 1009.4398

[Verdier 1967] J. L. Verdier, "The Lefschetz fixed point formula in etale cohomology", pp. 199-214 in Proc. Conf. Local Fields (Driebergen, 1966), edited by T. A. Springer, Springer, Berlin, 1967. MR 39 \#4173 Zbl 0169.52401

Communicated by Brian Conrad

Received 2010-07-08 Revised 2011-03-28 Accepted 2011-04-24

Shenghao.Sun@math.u-psud.fr Département de Mathématiques, Bâtiment 425,

Faculté des Sciences d'Orsay, Université Paris-Sud 11, F-91405 Orsay Cedex, France

http://www. math.u-psud.fr/ ssun/ 


\section{Algebra \& Number Theory}

msp.berkeley.edu/ant

\section{EDITORS}

MANAGING EDITOR

Bjorn Poonen

Massachusetts Institute of Technology

Cambridge, USA

\author{
EDITORIAL BOARD CHAIR \\ David Eisenbud \\ University of California \\ Berkeley, USA
}

\section{BOARD OF EDITORS}

Georgia Benkart

Dave Benson

Richard E. Borcherds

John H. Coates

J-L. Colliot-Thélène

Brian D. Conrad

Hélène Esnault

Hubert Flenner

Edward Frenkel

Andrew Granville

Joseph Gubeladze

Ehud Hrushovski

Craig Huneke

Mikhail Kapranov

Yujiro Kawamata

János Kollár

Yuri Manin

Barry Mazur

Philippe Michel

Susan Montgomery
University of Wisconsin, Madison, USA

University of Aberdeen, Scotland

University of California, Berkeley, USA

University of Cambridge, UK

CNRS, Université Paris-Sud, France

University of Michigan, USA

Universität Duisburg-Essen, Germany

Ruhr-Universität, Germany

University of California, Berkeley, USA

Université de Montréal, Canada

San Francisco State University, USA

Hebrew University, Israel

University of Kansas, USA

Yale University, USA

University of Tokyo, Japan

Princeton University, USA

Northwestern University, USA

Harvard University, USA

École Polytechnique Fédérale de Lausanne

University of Southern California, USA
Shigefumi Mori

Raman Parimala

Jonathan Pila

Victor Reiner

Karl Rubin

Peter Sarnak

Joseph H. Silverman

Michael Singer

Ronald Solomon

Vasudevan Srinivas

J. Toby Stafford

Bernd Sturmfels

Richard Taylor

Ravi Vakil

Michel van den Bergh

Marie-France Vignéras

Kei-Ichi Watanabe

Andrei Zelevinsky

Efim Zelmanov
RIMS, Kyoto University, Japan

Emory University, USA

University of Oxford, UK

University of Minnesota, USA

University of California, Irvine, USA

Princeton University, USA

Brown University, USA

North Carolina State University, USA

Ohio State University, USA

Tata Inst. of Fund. Research, India

University of Michigan, USA

University of California, Berkeley, USA

Harvard University, USA

Stanford University, USA

Hasselt University, Belgium

Université Paris VII, France

Nihon University, Japan

Northeastern University, USA

University of California, San Diego, USA

\section{PRODUCTION}

contact@msp.org

Silvio Levy, Scientific Editor

See inside back cover or www.jant.org for submission instructions.

The subscription price for 2012 is US \$175/year for the electronic version, and \$275/year (+\$40 shipping outside the US) for print and electronic. Subscriptions, requests for back issues from the last three years and changes of subscribers address should be sent to Mathematical Sciences Publishers, Department of Mathematics, University of California, Berkeley, CA 94720-3840, USA.

Algebra \& Number Theory (ISSN 1937-0652) at Mathematical Sciences Publishers, Department of Mathematics, University of California, Berkeley, CA 94720-3840 is published continuously online. Periodical rate postage paid at Berkeley, CA 94704, and additional mailing offices.

ANT peer review and production are managed by EditFLOW ${ }^{\circledR}$ from Mathematical Sciences Publishers.

PUBLISHED BY

mathematical sciences publishers

http://msp.org/

A NON-PROFIT CORPORATION

Typeset in IAT $_{\mathrm{E}} \mathrm{X}$

Copyright (C2012 by Mathematical Sciences Publishers 


\section{Algebra \& Number Theory}

\section{Volume $6 \quad$ No. $1 \quad 2012$}

The Chevalley-Shephard-Todd theorem for finite linearly reductive group schemes MATTHEW SATRIANO

The minimal resolution conjecture for points on del Pezzo surfaces

ROSA M. MIRÓ-ROIG and JOAN PONS-LLOPIS

$L$-series of Artin stacks over finite fields

SHENGHAO SUN

Multiplicative mimicry and improvements to the Pólya-Vinogradov inequality LEO GOLDMAKHER

Quiver Grassmannians and degenerate flag varieties

Giovanni Cerulli Irelli, Evgeny Feigin and Markus Reineke 